\title{
Atkinson Island Mooring Basin Alternatives, Houston Ship Channel
}

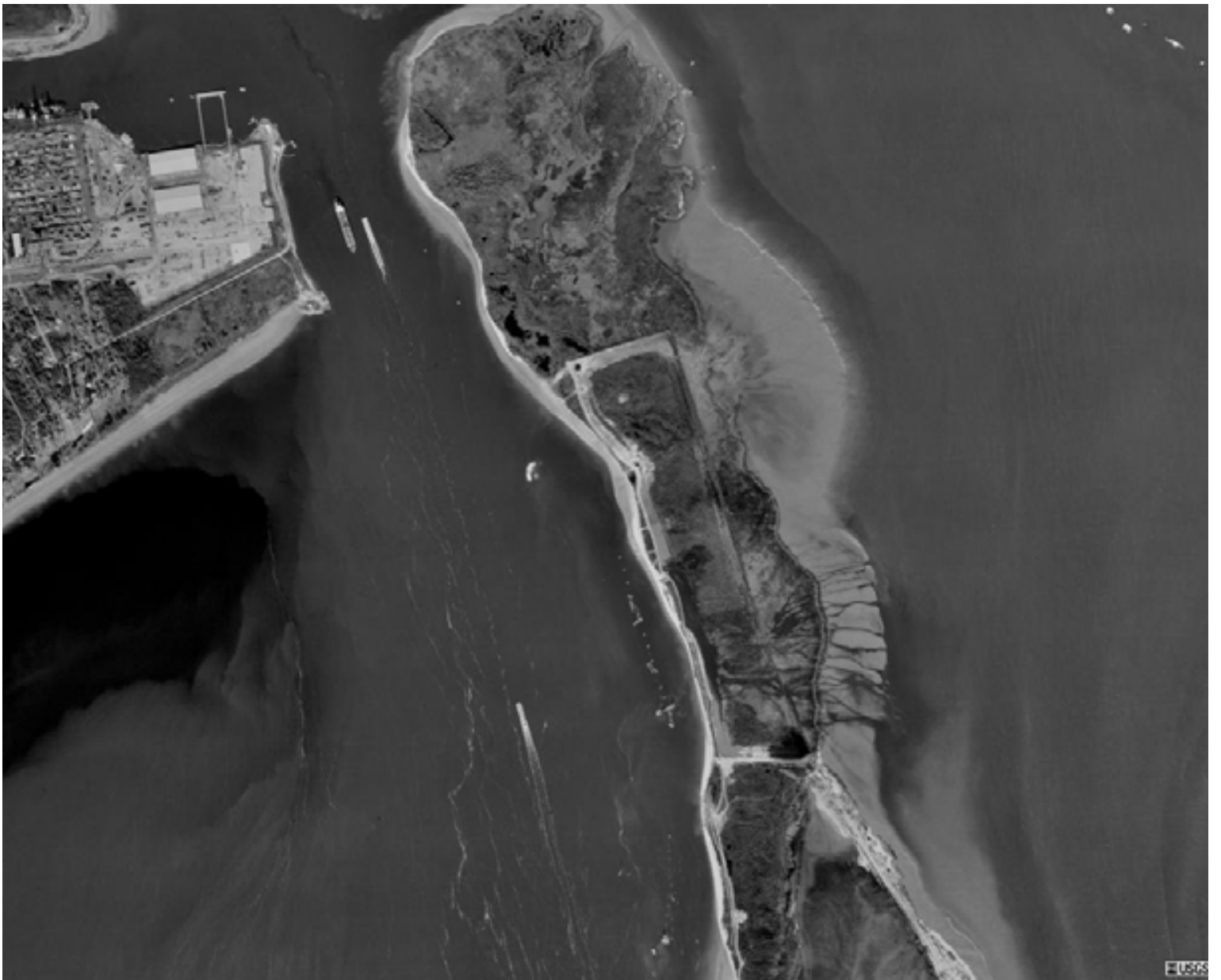




\section{Atkinson Island Mooring Basin Alternatives, Houston Ship Channel}

Stephen T. Maynord, John E. Hite, Jr., and Mario J. Sanchez

Coastal and Hydraulics Laboratory

U.S. Army Engineer Research and Development Center

3909 Halls Ferry Road

Vicksburg, MS 39180-6199

Final report

Approved for public release; distribution is unlimited. [or a restricted statement]

Prepared for U.S. Army Engineer District, Galveston

P.O. Box 1229

Galveston, TX 77553-1229 


\begin{abstract}
The U.S. Army Engineer District, Galveston (SWG), is investigating methods to reduce ship effects in the Atkinson Island Mooring Basin (AIMB) from ship traffic in the Houston Ship Channel. The Engineer Research and Development Center (ERDC) assisted SWG in this investigation. A field study was conducted first to gain an understanding of the ship effects that are causing mooring problems in the AIMB and collect ship effects data to be used to validate the numerical model HIVEL2D to the existing conditions in the Houston Ship Channel. The numerical model successfully reproduced the results from the field experiments and was then used to evaluate dike designs with the new AIMB channel. Several dike designs were evaluated and a 330o-ft-long dike with the centerline of the dike located $500 \mathrm{ft}$ west of the centerline of the AIMB channel was found to be effective in minimizing ship effects in the basin. End sections placed on the dike with a $150-\mathrm{ft}$ opening at the entrances to the basin further reduced the water-level variations inside the basin during ship transits. The dike design bw14 included these features and was recommended for installation at the project.
\end{abstract}

DISCLAIMER: The contents of this report are not to be used for advertising, publication, or promotional purposes. Citation of trade names does not constitute an official endorsement or approval of the use of such commercial products. All product names and trademarks cited are the property of their respective owners. The findings of this report are not to be construed as an official Department of the Army position unless so designated by other authorized documents. 


\section{Contents}

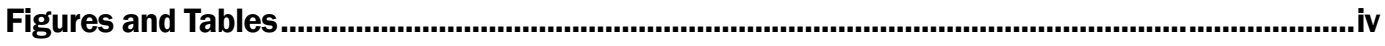

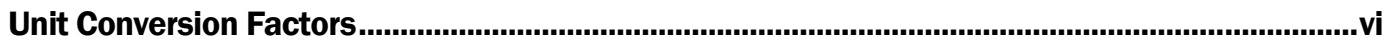

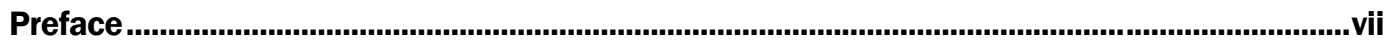

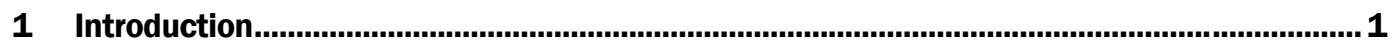

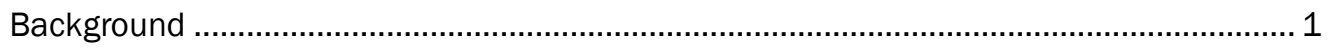

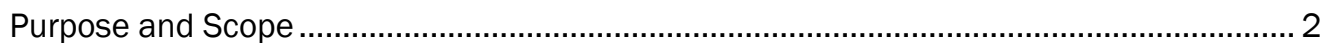

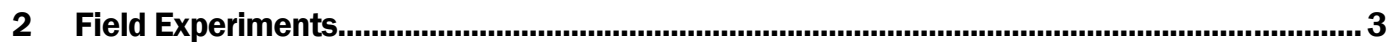

Gage Description

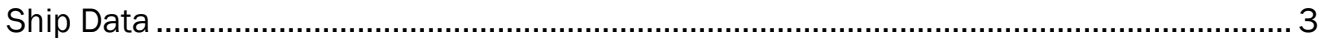

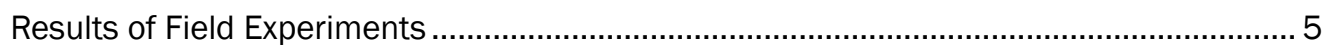

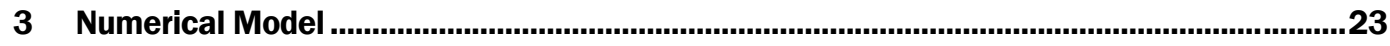

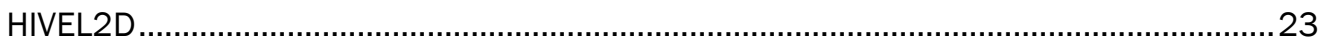

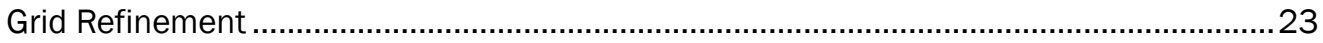

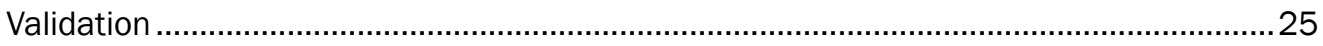

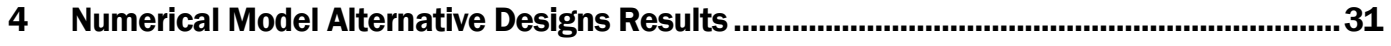

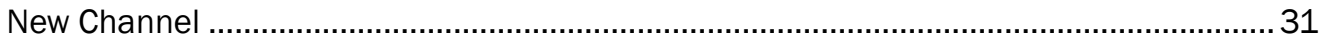

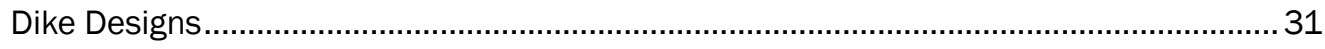

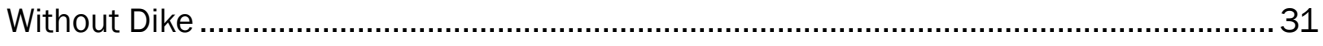

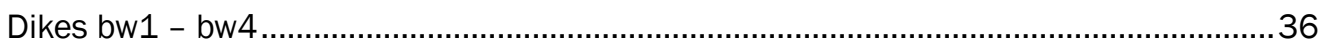

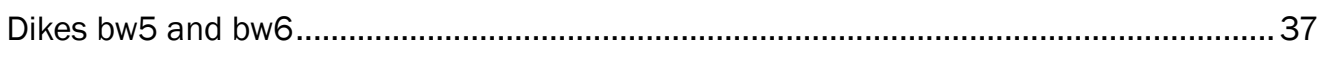

Dikes bw7 and bw8

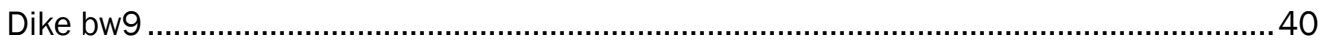

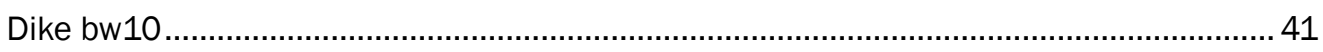

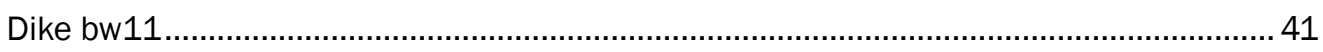

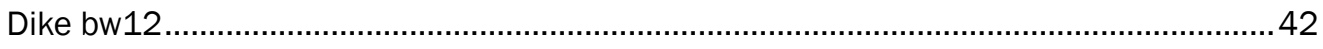

Dike bw13

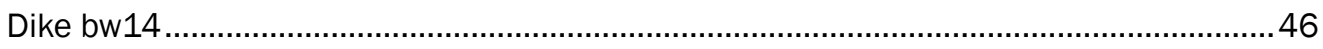

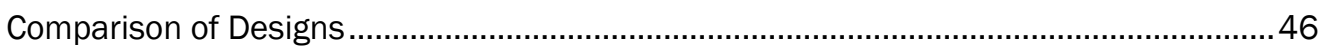

Dike bw14 with Tow Moored in Varying Locations ........................................................49

Dike bw14 with Different Ship Transits.................................................................... 49

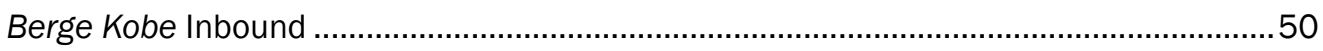

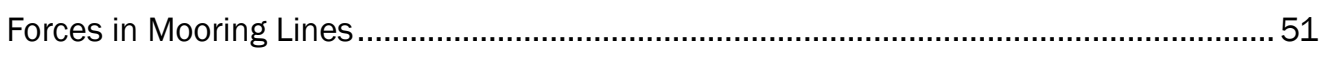

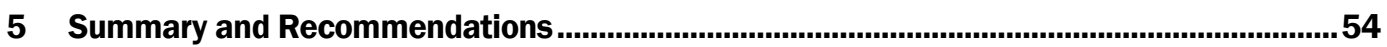

\section{Report Documentation Page}




\section{Figures and Tables}

\section{Figures}

Figure 1. Vicinity map and camera and gage location. ………...................................................... 1

Figure 2. Time histories at all water gages during inbound ship passages....................................... 12

Figure 3. Time histories of all gages during outbound ship passages............................................. 14

Figure 4. Berge Kobe, 4 June 2003 passage............................................................................... 16

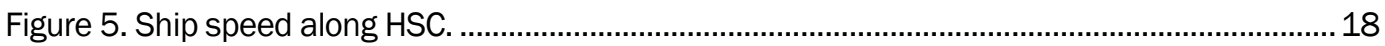

Figure 6. Detail of the original channel mesh. ............................................................................ 24

Figure 7. HIVEL2D computed water-level, SKS Trent, outbound, run 79565................................ 25

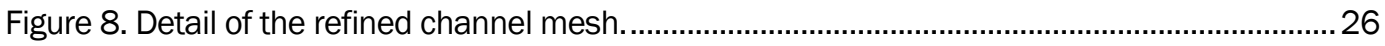

Figure 9. HIVEL2D computed water-level, SKS Trent, outbound, run 80561. ................................ 27

Figure 10. Detail of the refined mesh including the bay................................................................ 27

Figure 11. SKS Trent, outbound, all gages (computed - final model)..............................................2

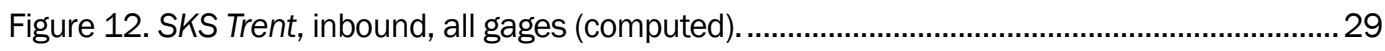

Figure 13. Eagle Subaru, outbound, all gages, (computed)..........................................................2

Figure 14. Berge Kobe, inbound, all gages (computed)............................................................. 30

Figure 15. Channel invert layout and mooring locations.....................................................................32

Figure 16. Water levels (ft) in basin without dike with a tow moored between E3 and E4

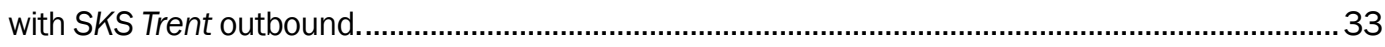

Figure 17. Water-level differences in basin without dike with tow moored at E3-E4, with SKS Trent outbound (Continued) ............................................................................................. 34

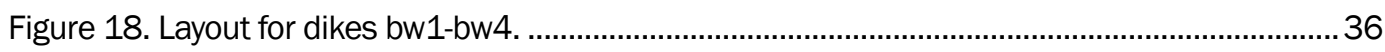

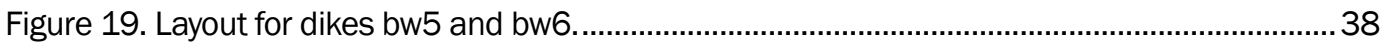

Figure 20. Water levels (ft) in basin with dike bw6 and with tow moored at E3-E4,

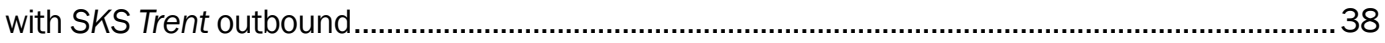

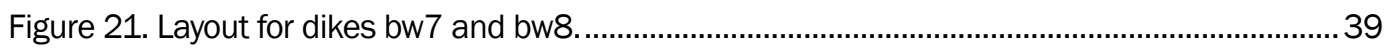

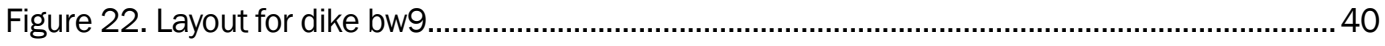

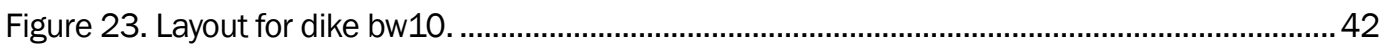

Figure 24. Layout for dike bw11. ............................................................................................. 43

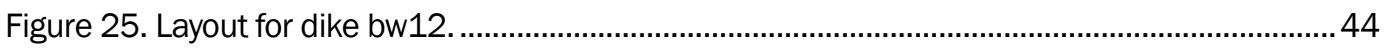

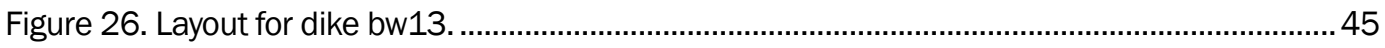

Figure 27. Layout for dike bw14..................................................................................................... 47

Figure 28. Water levels (ft) in basin with dike bw14 with tow moored at location E3-E4 and SKS Trent outbound.

Figure 29. Comparison of maximum water-level differences for the different dike designs with SKS Trent outbound.

Figure 30. Water levels (ft) in basin with dike bw14, Berge Kobe inbound and tow moored at E1-E2. 50

Figure 31. Water levels in basin with no dike and Berge Kobe inbound. 51 
Figure 32. Computed hawser forces for the different dike designs, SKS Trent outbound.................53

Figure 33. Time histories of computed hawser forces for the without dike and dike bw14 conditions, SKS Trent outbound.

\section{Tables}

Table 1. Pressure gages and camera locations. ……….............................................................. 4

Table 2. Ship details and timing from cameras............................................................................ 6

Table 3. Selected ships for data presentation.................................................................................... 11

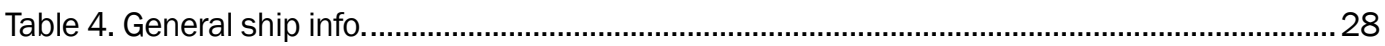

Table 5. Maximum water-level differences in mooring basin in feet, SKS Trent outbound................35

Table 6. Maximum water-level differences in mooring basin in feet with dike bw14 for

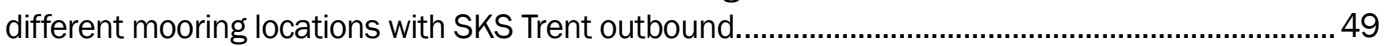

Table 7. Maximum water-level differences in mooring basin in feet with dike bw14 and barges moored in E3-E4 for different ship transits.

Table 8. Maximum water-level differences in mooring basin in feet without dike and barges moored in E3-E4 with Berge Kobe inbound. 


\section{Unit Conversion Factors}

\begin{tabular}{|l|c|l|}
\hline Multiply & By & To Obtain \\
\hline feet & 0.3048 & meters \\
\hline miles (U.S. statute) & $1,609.347$ & meters \\
\hline square feet & 0.09290304 & square meters \\
\hline tons (force) & $8,896.443$ & newtons \\
\hline tons (2,000 pounds, mass) & 907.1847 & kilograms \\
\hline
\end{tabular}




\section{Preface}

The investigation reported herein was authorized by the Headquarters, U.S. Army Corps of Engineers, at the request of U.S. Army Engineer District, Galveston, in June 2002. A field study was conducted in June 2003 and the remaining data analysis and numerical modeling was performed from June 2003 to October 2003 and October 2004 to October 2005. The field experiments were performed during 2-5 June 2003 by personnel of the Coastal and Hydraulics Laboratory (CHL) of the U.S. Army Engineer Research and Development Center (ERDC), under the general supervision of T. W. Richardson, Director, Coastal and Hydraulics Laboratory (CHL); Dr. W. D. Martin, Deputy Director, CHL; D. W. Webb, Acting Chief, Navigation Branch, CHL, and Drs. Sandra K. Knight and Jack E. Davis, Technical Directors, CHL.

The experimental and numerical program was led by Dr. S. T. Maynord, Navigation Branch, CHL, with the assistance T. Pratt and T. Waller, Field Data Collection and Analysis Branch, CHL, and S. W. Guy, Information Technology Laboratory, ERDC. Numerical model construction and simulations were performed by Dr. Maynord, Dr. J. E. Hite, Jr., and M. J. Sanchez. The report was written by Drs. Maynord and Hite. Dr. Richard Stockstill, CHL, performed a peer review of the report.

Bill Jakeway and Gerald Dunaway, U.S. Army Engineer District, Galveston, reviewed experiment results and discussed study results.

COL Richard B. Jenkins was Commander and Executive Director of ERDC. Dr. James R. Houston was Technical Director. 


\section{Introduction}

\section{Background}

The U.S. Army Engineer District, Galveston, proposes construction of a dike system to protect vessels moored in the Atkinson Island Mooring Basin (AIMB) from long period waves generated by ships transiting the Houston Ship Channel (HSC) adjacent to the mooring basin. The waves cause water-level variations throughout the mooring area, making it difficult for barges to remain moored. The AIMB is located southeast of Morgan's Point on the east side of the HSC, as shown in Figure 1.

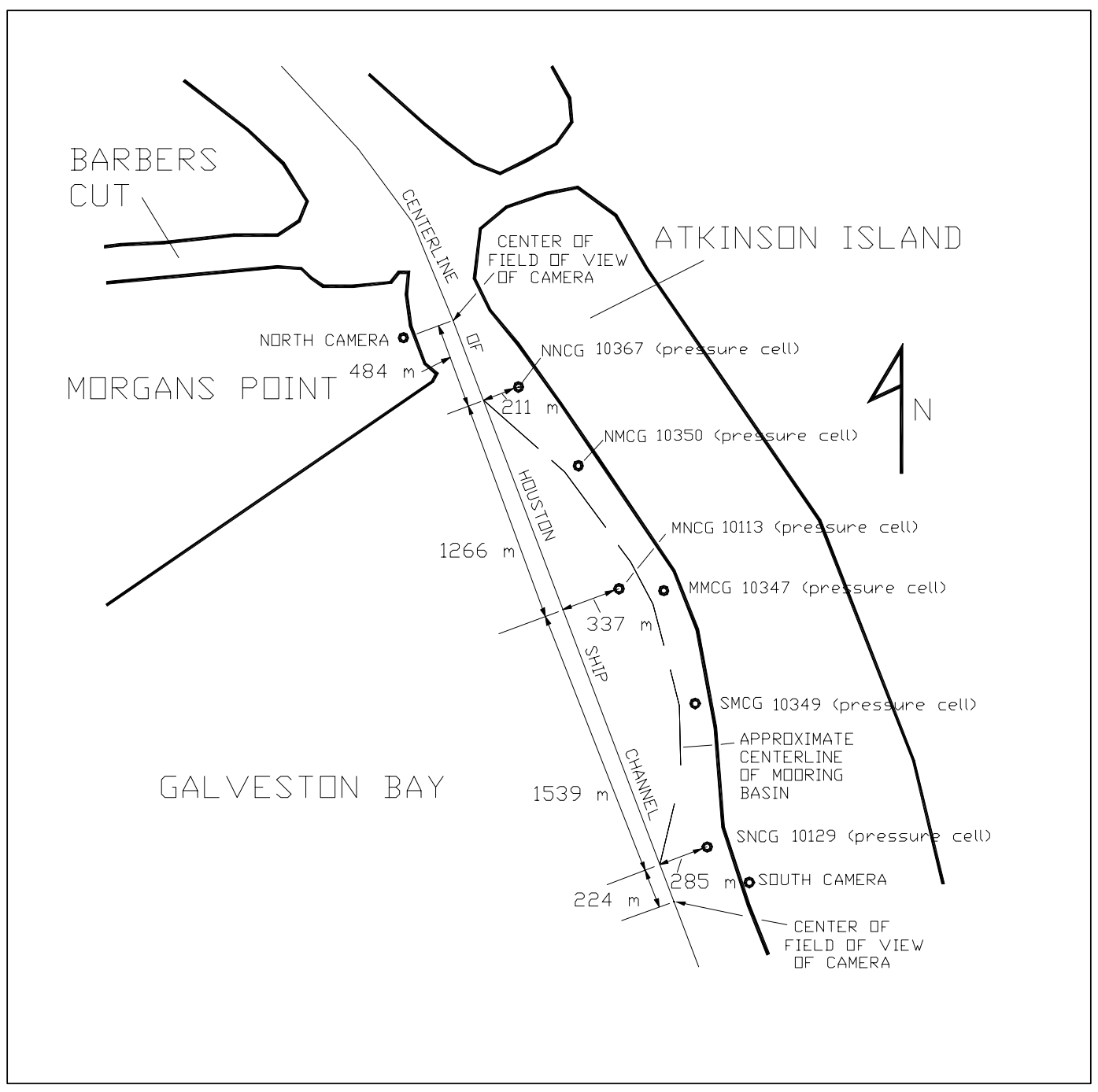

Figure 1. Vicinity map and camera and gage location. 


\section{Purpose and Scope}

This study evaluated alternative methods for minimizing water-surface variations inside the mooring basin during ship transits. If the water level in the mooring area can be kept fairly constant, the forces in the mooring lines will be reduced and the barges can remain moored. A field study was conducted first to gain an understanding of the ship effects that are causing mooring problems in the AIMB and to collect ship effects data to be used to validate the numerical model of the existing conditions in the HSC. Once validated, the existing geometry in the numerical model was changed to the deepened channel and barge mooring lanes for evaluation of alternatives. 


\section{Field Experiments}

\section{Gage Description}

Ship effects were measured along the HSC and along the AIMB from 2 to 5 June 2003. Six pressure cells were placed at selected locations in the navigation and mooring channels (Figure 1). The pressure cells were mounted on a conduit driven into the channel bottom. The sensors were mounted about $1.8 \mathrm{ft}$ above the bed in 5-7 ft of water; they were capable of capturing the long-period water-level changes caused by ship transits through the area. The 4 letter acronym used for each gage in Figure 1 is based on the following: 1) the first letter is North, Middle, or South; 2) the

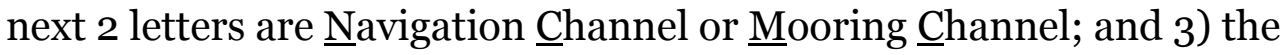
fourth letter $\mathrm{G}$ is for gage. The number following each four letter acronym is the serial number of the gage.

Figure 1 also shows the locations of two time lapse video cameras deployed at the north and south ends of the AIMB to determine ship speed based on ship length and time of passage at a fixed point on the video screen. Ship speed was also determined using the arrival time of the ship drawdown at the three gages along the HSC. All pressure cells and cameras were synchronized in time using Central Standard Time. Table 1 shows the State Plane coordinates (NAD 27 Texas South Central) of the pressure cells, the bed elevation at the pressure cells in Corps of Engineers Mean Low Tide (MLT), and the coordinates of the camera, and the center of the field of view of the camera at the center of the navigation channel. Typical water levels at the Morgan's Point tide gage average 2.0 ft above MLT and ranged from $0.9-2.7 \mathrm{ft}$ above MLT during the field study.

\section{Ship Data}

Ships using the HSC in the AIMB reach during the field study were 48 percent tankers, 21 percent bulk carriers, 16 percent container ships, 10 percent gas carriers, 2 percent ore boats, and 3 percent miscellaneous others. Ships vary in the amount of streamlining of the hull, which is indicated by their block coefficient. The block coefficient is equal to the underwater volume of the ship divided by (maximum length $\times$ maximum beam $\times$ maximum draft). The block coefficient would be 1.0 for a hypothetical ship having an unchanging rectangular cross section all along its length. 
Table 1. Pressure gages and camera locations.

\begin{tabular}{|l|l|l|l|}
\hline Pressure Gage & Coordinates, ft, North, East * & Bed Elevation, COE MLT, ft & Data rate, sec** \\
\hline NNCG 10367 & 692635,3276990 & -3.7 & 2 \\
\hline NMCG 10350 & 691177,3278095 & -3.5 & 1 \\
\hline MNCG 10113 & 688898,3278846 & -4.8 & 2 \\
\hline MMCG 10347 & 688863,3279672 & -3.8 & 1 \\
\hline SMCG 10349 & 686771,3280252 & -3.7 & 1 \\
\hline SNCG 10129 & 684115,3280472 & -4.4 & 2 \\
\hline North camera & 693771,3275384 & Not applicable & Not applicable \\
\hline Center of North camera view & 693875,3275782 & Not applicable & Not applicable \\
\hline South Camera & 683455,3281253 & Not applicable & Not applicable \\
\hline $\begin{array}{l}\text { Center of South camera view } \\
\text { * Coordinates are state plane, NAD 27, Texas South Central. } \\
\text { ** Gages along the navigation channel sampled the water level every 2 seconds. Gages along the mooring } \\
\text { channel sampled the water level every 1 second. }\end{array}$ & Not applicable \\
\hline
\end{tabular}

Ships whose primary goal is moving material with a sacrifice in speed have block coefficients of 0.75 to 0.85 and include tankers, bulk carriers, and gas carriers. The Berge Kobe, one of the gas carriers on the HSC, has a block coefficient of 0.79 . Ships having a goal of moving material while maintaining relatively high speeds have block coefficients of about 0.55 to 0.65 and are primarily container ships on the HSC. The TMM Hermosilla is one of the container ships on the HSC that has a block coefficient of o.6o. The importance of this discussion is that container ships, because of their more streamlined shape, generally require a greater speed to produce the same effects as a less streamlined ship.

An important factor affecting speed of container ships in the AIMB reach is that almost every container ship on the HSC berths at Barbers Cut, located just North of Morgan's Point (Figure 1). Both inbound and outbound container ships require tug assistance to enter and exit Barbers Cut. For outbound container ships, the tugs detach from the ship at or just south of Morgan's Point and they can reach significant speeds at the lower end of the mooring basin. For inbound container ships, the tugs generally meet the ships somewhere between the south end and the middle of the mooring basin. The tug assist to the inbound container ship causes a major reduction of their speed along the AIMB. While some of the inbound container ships had relatively high speeds at the south camera, speeds were reduced by the time the ship reached the middle of the AIMB. 
Table 2 shows the list of ships using the HSC during the field study along with pertinent details of the ship, arrival times at the cameras, and derived speeds from the cameras. Development of the ship log in Table 2 was enormously helped by the company Marine Exchange of the West Gulf, Inc. They have an office at Morgan's Point that records the name, time of passage, type, and direction of every ship passing Morgan's Point. Table 2 shows the ship type designation used by the Marine Exchange. Also shown in Table 2 is whether another ship was in the AIMB reach going in the opposite direction in what is referred to as a meeting maneuver.

In the water-level data collected in the field study, the largest water-level variations occurred with isolated ships traveling at high speed as opposed to meeting ships traveling at reduced speeds. The numerical model in this study will be validated, and the subsequent testing of alternatives will be conducted with isolated ships traveling at high speed. Ships were selected from the Table 2 ships to provide water-level data for this report and to use in the validation phase of this study. Criteria for selection were as follows: 1) no meeting of other ships in the AIMB reach; 2) range of ship types; 3) ships having the largest effects, which generally means the largest, fastest ships; and 4) inbound and outbound ships.

\section{Results of Field Experiments}

Selected ships are shown in Table 3 along with the figure numbers for the water-level data described in the subsequent sections. Each gage is plotted relative to the average ambient water level determined over a period of 5 minutes prior to the arrival of the ship effects. The time-histories of water level shown on the figures with all gages (Figures 2 and 3) have each gage offset by $2.0 \mathrm{ft}$ for clarity. Individual gage plots are shown for the Berge Kobe in Figure 4 for the navigation channel gages and show the position of the bow and stern of the ship relative to the water-level variations. 
Table 2. Ship details and timing from cameras.

\begin{tabular}{|c|c|c|c|c|c|c|c|c|c|c|c|c|c|c|}
\hline Ship Name & $\begin{array}{l}\text { Length } \\
\mathrm{ft}\end{array}$ & $\begin{array}{l}\text { Beam } \\
\mathrm{ft}\end{array}$ & $\begin{array}{l}\text { Draft } \\
\mathrm{ft}\end{array}$ & Date & Direction & $\begin{array}{l}\text { Ship } \\
\text { Type }\end{array}$ & $\begin{array}{l}\text { Time- } \\
\text { MP log }\end{array}$ & $\begin{array}{l}\text { Time** } \\
\text { at NC }\end{array}$ & $\begin{array}{l}\text { B-S } \\
\text { at NC } \\
\text { sec }\end{array}$ & $\begin{array}{l}\text { Time** } \\
\text { at SC }\end{array}$ & $\begin{array}{l}\text { B-S } \\
\text { at SC } \\
\text { sec }\end{array}$ & $\begin{array}{l}\text { Meeting } \\
\text { Ships }\end{array}$ & $\begin{array}{l}\text { NC } \\
\text { Speed } \\
\text { fps }\end{array}$ & $\begin{array}{l}\text { SC } \\
\text { Speed } \\
\text { fps }\end{array}$ \\
\hline \multirow[t]{2}{*}{ Norgas Discoverer } & 411 & 62 & 25 & $6 / 2 / 2003$ & OUT & LN & $0: 00$ & 10:06:35* & 24 & & & & 17.13 & \\
\hline & & & & & & & & 10:06:59* & & & & & & \\
\hline \multirow[t]{2}{*}{ Bro Stella } & 700 & 118 & 25 & $6 / 2 / 2003$ & OUT & TK & 1120 & $10: 22: 35 *$ & 49 & & & & 14.29 & \\
\hline & & & & & & & & 10:23:24* & & & & & & \\
\hline \multirow[t]{2}{*}{ Panam Felice } & 385 & 65 & 23 & $6 / 2 / 2003$ & IN & CT & 1450 & 13:53:20* & 21 & 13:43:36 & 18 & $N$ & 18.33 & 21.39 \\
\hline & & & & & & & & 13:53:41* & & 13:43:54 & & & & \\
\hline \multirow[t]{2}{*}{ Yick Luk } & 623 & 103 & 23 & $6 / 2 / 2003$ & IN & BB & 1505 & 14:03:37* & 33 & 13:53:47 & 32 & $N$ & 18.88 & 19.47 \\
\hline & & & & & & & & 14:04:10* & & 13:54:19 & & & & \\
\hline \multirow[t]{2}{*}{ Zemgale } & 750 & 106 & 40 & $6 / 2 / 2003$ & IN & TK & 1615 & 15:17:33 & 67 & 15:03:33 & 50 & $N$ & 11.19 & 15.00 \\
\hline & & & & & & & & 15:18:40 & & 15:04:23 & & & & \\
\hline \multirow[t]{2}{*}{ Isabel Knutsen } & 524 & 76 & 32 & $6 / 2 / 2003$ & OUT & CT & 1650 & 15:52:03 & 30 & $16: 02: 00$ & 25 & $\mathrm{Y}$ & 17.47 & 20.96 \\
\hline & & & & & & & & $15: 52: 33$ & & $16: 02: 25$ & & & & \\
\hline \multirow[t]{2}{*}{ Northern Snow } & 431 & 66 & 27 & $6 / 2 / 2003$ & IN & LN & 1700 & 15:59:03 & 27 & 15:48:26 & 21 & Y & 15.96 & 20.52 \\
\hline & & & & & & & & 15:59:30 & & $15: 48: 47$ & & & & \\
\hline \multirow[t]{2}{*}{ Maersk San Juan } & 489 & 76 & 22 & $6 / 2 / 2003$ & OUT & CS & 1840 & $17: 37: 34$ & 32 & $17: 46: 20$ & 20 & $N$ & 15.28 & 24.45 \\
\hline & & & & & & & & 17:38:06 & & $17: 46: 40$ & & & & \\
\hline \multirow[t]{2}{*}{ Milagro } & 722 & 116 & 34 & $6 / 2 / 2003$ & OUT & TK & 2015 & 19:12:48 & 53 & 19:25:59 & 45 & $N$ & 13.62 & 16.04 \\
\hline & & & & & & & & 19:13:41 & & 19:26:44 & & & & \\
\hline \multirow[t]{2}{*}{ Senang Spirit } & 810 & 138 & 28 & $6 / 2 / 2003$ & OUT & TK & 2045 & 19:48:41 & 60 & 20:00:38 & 46 & $N$ & 13.50 & 17.61 \\
\hline & & & & & & & & 19:49:41 & & 20:01:24 & & & & \\
\hline \multirow[t]{2}{*}{ Sea Admiral } & 569 & 76 & 25 & $6 / 2 / 2003$ & IN & RO & 2115 & 20:13:50 & 44 & 20:02:35 & 32 & $N$ & 12.93 & 17.78 \\
\hline & & & & & & & & 20:14:34 & & 20:03:07 & & & & \\
\hline \multirow[t]{2}{*}{ Domenico levoli } & 479 & 71 & 23 & $6 / 2 / 2003$ & IN & CT & 2120 & 20:20:43 & 31 & 20:09:03 & 30 & $N$ & 15.45 & 15.97 \\
\hline & & & & & & & & 20:21:14 & & 20:09:33 & & & & \\
\hline \multirow[t]{2}{*}{ SCM Alexander } & 414 & 66 & 27 & $6 / 2 / 2003$ & IN & BB & 2200 & 21:00:47 & 28 & $20: 48: 56$ & 24 & $N$ & 14.79 & 17.25 \\
\hline & & & & & & & & 21:01:15 & & $20: 49: 20$ & & & & \\
\hline \multirow[t]{2}{*}{ Altair } & 490 & 78 & 23 & $6 / 2 / 2003$ & IN & CT & 2215 & 21:15:08 & 35 & 21:03:48 & 27 & $N$ & 14.00 & 18.15 \\
\hline & & & & & & & & 21:15:43 & & 21:04:15 & & & & \\
\hline \multirow[t]{2}{*}{ Norgas Sonoma } & 410 & 65 & 26 & $6 / 2 / 2003$ & IN & LN & 2350 & 22:48:33 & 22 & 22:39:53 & 18 & $N$ & 18.64 & 22.78 \\
\hline & & & & & & & & 22:48:55 & & 22:40:11 & & & & \\
\hline \multirow[t]{2}{*}{ Rio Blanco } & 654 & 98 & 29 & $6 / 2 / 2003$ & IN & VC & 15 & 23:14:17 & 39 & 23:03:46 & 35 & $N$ & 16.77 & 18.69 \\
\hline & & & & & & & & 23:14:56 & & 23:04:21 & & & & \\
\hline \multirow[t]{2}{*}{ Sea Orchid } & 610 & 99 & 21 & $6 / 2 / 2003$ & OUT & $B C$ & 30 & 23:33:54 & 44 & 23:43:36 & 22 & $N$ & 13.86 & 27.73 \\
\hline & & & & & & & & 23:34:38 & & 23:43:58 & & & & \\
\hline \multirow[t]{2}{*}{ Patriot } & 580 & 96 & 21 & $6 / 3 / 2003$ & IN & $B C$ & 130 & 0:29:29 & 32 & $0: 19: 21$ & 28 & $N$ & 18.13 & 20.71 \\
\hline & & & & & & & & 0:30:01 & & $0: 19: 49$ & & & & \\
\hline
\end{tabular}




\begin{tabular}{|c|c|c|c|c|c|c|c|c|c|c|c|c|c|c|}
\hline Ship Name & $\begin{array}{l}\text { Length } \\
\mathrm{ft}\end{array}$ & $\begin{array}{l}\text { Beam } \\
\mathrm{ft}\end{array}$ & $\begin{array}{l}\text { Draft } \\
\mathrm{ft}\end{array}$ & Date & Direction & $\begin{array}{l}\text { Ship } \\
\text { Type }\end{array}$ & \begin{tabular}{|l} 
Time- \\
MP log
\end{tabular} & $\begin{array}{l}\text { Time** } \\
\text { at NC }\end{array}$ & \begin{tabular}{|l} 
B-S \\
at NC \\
sec
\end{tabular} & $\begin{array}{l}\text { Time** } \\
\text { at SC }\end{array}$ & $\begin{array}{l}\text { B-S } \\
\text { at SC } \\
\text { sec }\end{array}$ & $\begin{array}{l}\text { Meeting } \\
\text { Ships }\end{array}$ & $\begin{array}{l}\text { NC } \\
\text { Speed } \\
\text { fps }\end{array}$ & $\begin{array}{l}\text { SC } \\
\text { Speed } \\
\text { fps }\end{array}$ \\
\hline \multirow[t]{2}{*}{ Prodicos } & 631 & 101 & 33 & $6 / 3 / 2003$ & IN & TK & 255 & 1:52:34 & 45 & 1:40:16 & 38 & $N$ & 14.02 & 16.61 \\
\hline & & & & & & & & 1:53:19 & & 1:40:54 & & & & \\
\hline \multirow[t]{2}{*}{ TMM Hermosillo } & 885 & 106 & 32 & $6 / 3 / 2003$ & OUT & CS & 320 & $2: 16: 53$ & 91 & 2:29:39 & 44 & $N$ & 9.73 & 20.11 \\
\hline & & & & & & & & 2:18:24 & & $2: 30: 23$ & & & & \\
\hline \multirow[t]{2}{*}{ Sea-Land Value } & 856 & 106 & 30 & $6 / 3 / 2003$ & OUT & CS & 520 & 4:19:00 & 89 & 4:32:07 & 48 & $Y$ & 9.62 & 17.83 \\
\hline & & & & & & & & 4:20:29 & & $4: 32: 55$ & & & & \\
\hline \multirow[t]{2}{*}{ CEC Mirage } & 331 & 63 & 24 & $6 / 3 / 2003$ & IN & BB & 530 & 4:29:50 & 15 & $4: 20: 44$ & 14 & $Y$ & 22.07 & 23.64 \\
\hline & & & & & & & & 4:30:05 & & $4: 20: 58$ & & & & \\
\hline \multirow[t]{2}{*}{\begin{tabular}{|l|} 
Sea-Land \\
Voyager $(T)$ \\
\end{tabular}} & 845 & 101 & 25 & $6 / 3 / 2003$ & IN & CS & 610 & 5:16:10 & 110 & 4:55:07 & 70 & $N$ & 7.68 & 12.07 \\
\hline & & & & & & & & 5:18:00 & & $4: 56: 17$ & & & & \\
\hline \multirow[t]{2}{*}{ Saudi Diriyah } & 816 & 106 & 30 & $6 / 3 / 2003$ & OUT & $\mathrm{CP}$ & 640 & 5:39:15 & 74 & 5:50:30 & 39 & $Y$ & 11.03 & 20.92 \\
\hline & & & & & & & & 5:40:29 & & 5:51:09 & & & & \\
\hline \multirow[t]{2}{*}{ UBC Barranquilla } & 517 & 82 & 30 & $6 / 3 / 2003$ & IN & $\mathrm{BC}$ & 650 & 5:50:03 & 39 & 5:36:30 & 33 & $Y$ & 13.26 & 15.67 \\
\hline & & & & & & & & 5:50:42 & & 5:37:03 & & & & \\
\hline \multirow[t]{2}{*}{ Lofoten } & 810 & 138 & 29 & $6 / 3 / 2003$ & OUT & TK & 720 & $6: 23: 54$ & 68 & $6: 36: 45$ & 48 & $Y$ & 11.91 & 16.88 \\
\hline & & & & & & & & $6: 25: 02$ & & $6: 37: 33$ & & & & \\
\hline \multirow[t]{2}{*}{ Ionia } & 610 & 93 & 22 & $6 / 3 / 2003$ & IN & $B C$ & 730 & 6:31:36 & 28 & $6: 22: 44$ & 28 & $Y$ & 21.79 & 21.79 \\
\hline & & & & & & & & 6:32:04 & & $6: 23: 12$ & & & & \\
\hline \multirow[t]{2}{*}{ Panam Felice } & 385 & 65 & 26 & $6 / 3 / 2003$ & OUT & CT & 730 & 6:31:22 & 36 & $6: 45: 46$ & 36 & $\mathrm{~N}$ & 10.69 & 10.69 \\
\hline & & & & & & & & $6: 31: 58$ & & $6: 46: 22$ & & & & \\
\hline \multirow[t]{2}{*}{ TMM Colima } & 799 & 106 & 30 & $6 / 3 / 2003$ & OUT & CS & 750 & $6: 47: 36$ & 206 & 7:01:51 & 42 & $Y$ & 3.88 & 19.02 \\
\hline & & & & & & & & 6:51:02 & & 7:02:33 & & & & \\
\hline \multirow[t]{2}{*}{ Stolt Sapphire } & 580 & 106 & 39 & $6 / 3 / 2003$ & OUT & CT & 800 & 7:00:52 & 60 & 7:16:07 & 39 & $Y$ & 9.67 & 14.87 \\
\hline & & & & & & & & 7:01:52 & & 7:16:46 & & & & \\
\hline \multirow[t]{2}{*}{ Ikan Sepat } & 590 & 75 & 20 & $6 / 3 / 2003$ & IN & $B C$ & 805 & 7:05:00 & 37 & 6:54:08 & 30 & $Y$ & 15.95 & 19.67 \\
\hline & & & & & & & & 7:05:37 & & 6:54:38 & & & & \\
\hline \multirow[t]{2}{*}{ Zim Mexico III } & 534 & 73 & 25 & $6 / 3 / 2003$ & OUT & CS & 820 & 7:22:03 & 35 & 7:32:24 & 29 & $Y$ & 15.26 & 18.41 \\
\hline & & & & & & & & $7: 22: 38$ & & $7: 32: 53$ & & & & \\
\hline \multirow[t]{2}{*}{ Patsy N (T) } & 617 & 99 & 27 & $6 / 3 / 2003$ & IN & CS & 845 & 7:46:07 & 93 & 7:28:10 & 42 & $Y$ & 6.63 & 14.69 \\
\hline & & & & & & & & $7: 47: 40$ & & $7: 28: 52$ & & & & \\
\hline \multirow[t]{2}{*}{$\begin{array}{l}\text { Coral } \\
\text { Pavona(Baytk2) }\end{array}$} & 378 & 55 & 24 & $6 / 3 / 2003$ & OUT & LN & 915 & $8: 12: 43$ & 36 & 8:24:20 & 20 & $N$ & 10.50 & 18.90 \\
\hline & & & & & & & & $8: 13: 19$ & & 8:24:40 & & & & \\
\hline \multirow[t]{2}{*}{ Lykes Discoverer } & 850 & 106 & 31 & $6 / 3 / 2003$ & OUT & CS & 1015 & 9:13:51 & 111 & 9:28:30 & 73 & $N$ & 7.66 & 11.64 \\
\hline & & & & & & & & 9:15:42 & & 9:29:43 & & & & \\
\hline
\end{tabular}




\begin{tabular}{|c|c|c|c|c|c|c|c|c|c|c|c|c|c|c|}
\hline Ship Name & $\begin{array}{l}\text { Length } \\
\mathrm{ft}\end{array}$ & $\begin{array}{l}\text { Beam } \\
\mathrm{ft}\end{array}$ & $\begin{array}{l}\text { Draft } \\
\mathrm{ft}\end{array}$ & Date & Direction & $\begin{array}{l}\text { Ship } \\
\text { Type }\end{array}$ & $\begin{array}{l}\text { Time- } \\
\text { MP log }\end{array}$ & $\begin{array}{l}\text { Time** } \\
\text { at NC }\end{array}$ & $\begin{array}{l}\text { B-S } \\
\text { at NC } \\
\text { sec }\end{array}$ & $\begin{array}{l}\text { Time** } \\
\text { at SC }\end{array}$ & $\begin{array}{l}\text { B-S } \\
\text { at SC } \\
\text { sec }\end{array}$ & $\begin{array}{l}\text { Meeting } \\
\text { Ships }\end{array}$ & $\begin{array}{l}\text { NC } \\
\text { Speed } \\
\text { fps }\end{array}$ & $\begin{array}{l}\text { SC } \\
\text { Speed } \\
\text { fps }\end{array}$ \\
\hline \multirow[t]{2}{*}{ Golden Jane } & 488 & 72 & 30 & $6 / 3 / 2003$ & OUT & CT & 1045 & 9:44:20 & 31 & 9:56:39 & 34 & $Y$ & 15.74 & 14.35 \\
\hline & & & & & & & & 9:44:51 & & $9: 57: 13$ & & & & \\
\hline \multirow[t]{2}{*}{ Torm Ann-Marie } & 800 & 138 & 40 & $6 / 3 / 2003$ & IN & TK & 1055 & $9: 56: 13$ & 60 & 9:43:08 & 51 & $Y$ & 13.33 & 15.69 \\
\hline & & & & & & & & $9: 57: 13$ & & 9:43:59 & & & & \\
\hline \multirow[t]{2}{*}{ Sanko Quality } & 810 & 138 & 38 & $6 / 3 / 2003$ & IN & TK & 1125 & 10:27:43 & 65 & 10:13:55 & 56 & $N$ & 12.46 & 14.46 \\
\hline & & & & & & & & 10:28:48 & & $10: 14: 51$ & & & & \\
\hline \multirow[t]{2}{*}{$\begin{array}{l}\text { Lykes } \\
\text { Ambassador (T) }\end{array}$} & 889 & 106 & 36 & $6 / 3 / 2003$ & IN & CS & 1515 & 14:18:25 & 112 & 14:03:27 & 50 & $N$ & 7.94 & 17.78 \\
\hline & & & & & & & & 14:20:17 & & 14:04:17 & & & & \\
\hline \multirow[t]{2}{*}{ SKS Trent } & 800 & 138 & 32 & $6 / 3 / 2003$ & IN & OB & 1540 & 14:40:26 & 60 & 14:28:05 & 49 & $N$ & 13.33 & 16.33 \\
\hline & & & & & & & & 14:41:26 & & $14: 28: 54$ & & & & \\
\hline \multirow[t]{2}{*}{ Jo Sypress } & 598 & 105 & 28 & $6 / 3 / 2003$ & IN & CT & 1615 & 15:17:15 & 41 & 15:06:25 & 29 & $N$ & 14.59 & 20.62 \\
\hline & & & & & & & & 15:17:56 & & 15:06:54 & & & & \\
\hline \multirow[t]{2}{*}{ Eagle Subaru } & 810 & 138 & 28 & $6 / 3 / 2003$ & OUT & TK & 1650 & 15:48:40 & 50 & 15:59:57 & 44 & $\mathrm{~N}$ & 16.20 & 18.41 \\
\hline & & & & & & & & 15:49:30 & & 16:00:41 & & & & \\
\hline \multirow[t]{2}{*}{ BBC Iceland } & 330 & 54 & 16 & $6 / 3 / 2003$ & OUT & BB & 1805 & 17:09:18 & 14 & $17: 17: 30$ & 14 & $N$ & 23.57 & 23.57 \\
\hline & & & & & & & & 17:09:32 & & $17: 17: 44$ & & & & \\
\hline \multirow[t]{2}{*}{ Altair } & 490 & 78 & 23 & $6 / 3 / 2003$ & OUT & CT & 2025 & 19:24:30 & 25 & 19:33:30 & 22 & $N$ & 19.60 & 22.27 \\
\hline & & & & & & & & 19:24:55 & & 19:33:52 & & & & \\
\hline \multirow[t]{2}{*}{ Marion Green } & 469 & 71 & 21 & $6 / 3 / 2003$ & OUT & BB & 2105 & 20:06:03 & 22 & $20: 14: 32$ & 25 & $N$ & 21.32 & 18.76 \\
\hline & & & & & & & & 20:06:25 & & $20: 14: 57$ & & & & \\
\hline \multirow[t]{2}{*}{ Igloo Moon } & 465 & 72 & 20 & $6 / 3 / 2003$ & IN & LN & 2105 & 20:02:26 & 29 & $19: 52: 44$ & 20 & $N$ & 16.03 & 23.25 \\
\hline & & & & & & & & 20:02:55 & & 19:53:04 & & & & \\
\hline \multirow[t]{2}{*}{ SCM Alexander } & 414 & 66 & 24 & $6 / 3 / 2003$ & OUT & BB & 2125 & 20:23:25 & 25 & $20: 34: 16$ & 21 & $N$ & 16.56 & 19.71 \\
\hline & & & & & & & & 20:23:50 & & $20: 34: 37$ & & & & \\
\hline \multirow[t]{2}{*}{ BBC Anglia } & 333 & 54 & 20 & $6 / 3 / 2003$ & OUT & BB & 2150 & 20:49:23 & 14 & $20: 57: 49$ & 15 & $N$ & 23.79 & 22.20 \\
\hline & & & & & & & & 20:49:37 & & 20:58:04 & & & & \\
\hline \multirow[t]{2}{*}{ Domenico levoli } & 479 & 71 & 27 & $6 / 3 / 2003$ & OUT & CT & 2255 & 21:51:30 & 36 & 22:06:28 & 40 & $N$ & 13.31 & 11.98 \\
\hline & & & & & & & & 21:52:06 & & 22:07:08 & & & & \\
\hline \multirow[t]{2}{*}{ Team Merkur } & 605 & 99 & 32 & $6 / 3 / 2003$ & IN & CT & 2330 & 22:29:36 & 47 & $22: 17: 23$ & 37 & $N$ & 12.87 & 16.35 \\
\hline & & & & & & & & 22:30:23 & & 22:18:00 & & & & \\
\hline \multirow[t]{2}{*}{ SAF Marine Bioko } & 521 & 79 & 27 & $6 / 3 / 2003$ & OUT & BB & 25 & 23:23:47 & 27 & 23:33:34 & 24 & $N$ & 19.30 & 21.71 \\
\hline & & & & & & & & 23:24:14 & & 23:33:58 & & & & \\
\hline \multirow[t]{2}{*}{ Panam Atlantico } & 442 & 68 & 24 & $6 / 3 / 2003$ & IN & CT & 50 & 23:47:08 & 26 & 23:37:08 & 20 & $N$ & 17.00 & 22.10 \\
\hline & & & & & & & & 23:47:34 & & 23:37:28 & & & & \\
\hline \multirow[t]{2}{*}{ Zemgale } & 750 & 106 & 32 & $6 / 4 / 2003$ & OUT & TK & 115 & $0: 14: 24$ & 49 & 0:26:08 & 40 & $N$ & 15.31 & 18.75 \\
\hline & & & & & & & & $0: 15: 13$ & & $0: 26: 48$ & & & & \\
\hline
\end{tabular}




\begin{tabular}{|c|c|c|c|c|c|c|c|c|c|c|c|c|c|c|}
\hline Ship Name & $\begin{array}{l}\text { Length } \\
\mathrm{ft}\end{array}$ & $\begin{array}{l}\text { Beam } \\
\mathrm{ft}\end{array}$ & $\begin{array}{l}\text { Draft } \\
\mathrm{ft}\end{array}$ & Date & Direction & $\begin{array}{l}\text { Ship } \\
\text { Type }\end{array}$ & $\begin{array}{l}\text { Time- } \\
\text { MP log }\end{array}$ & $\begin{array}{l}\text { Time**} \\
\text { at NC }\end{array}$ & $\begin{array}{l}\text { B-S } \\
\text { at NC } \\
\text { sec }\end{array}$ & $\begin{array}{l}\text { Time**} \\
\text { at SC }\end{array}$ & $\begin{array}{l}\text { B-S } \\
\text { at SC } \\
\text { sec }\end{array}$ & $\begin{array}{l}\text { Meeting } \\
\text { Ships }\end{array}$ & $\begin{array}{l}\text { NC } \\
\text { Speed } \\
\text { fps }\end{array}$ & $\begin{array}{l}\text { SC } \\
\text { Speed } \\
\text { fps }\end{array}$ \\
\hline \multirow[t]{2}{*}{ Patsy N (T) } & 617 & 99 & 25.5 & $6 / 4 / 2003$ & OUT & CS & 135 & 0:38:02 & 76 & $0: 55: 13$ & 43 & $N$ & 8.12 & 14.35 \\
\hline & & & & & & & & 0:39:28 & & $0: 55: 56$ & & & & \\
\hline \multirow[t]{2}{*}{$\begin{array}{l}\text { Sea-Land Voyager } \\
(T)\end{array}$} & 845 & 101 & 26 & $6 / 4 / 2003$ & OUT & CS & 225 & 1:22:04 & 100 & 1:35:01 & 45 & $Y$ & 8.45 & 18.78 \\
\hline & & & & & & & & 1:23:44 & & 1:35:46 & & & & \\
\hline \multirow[t]{2}{*}{ MSC Zrin $(T)$} & 661 & 107 & 28 & $6 / 4 / 2003$ & IN & CS & 235 & 1:33:46 & 22 & 1:17:46 & 25 & Y & 30.05 & 26.44 \\
\hline & & & & & & & & 1:34:08 & & 1:18:11 & & & & \\
\hline \multirow[t]{2}{*}{ Tamara } & 810 & 139 & 28 & $6 / 4 / 2003$ & OUT & TK & 740 & 6:41:59 & 70 & 6:55:34 & 51 & Y & 11.57 & 15.88 \\
\hline & & & & & & & & 6:43:09 & & $6: 56: 25$ & & & & \\
\hline \multirow[t]{2}{*}{ Elsa } & 350 & 59 & 14 & $6 / 4 / 2003$ & IN & BB & 800 & 6:59:25 & 21 & 6:50:09 & 16 & Y & 16.67 & 21.88 \\
\hline & & & & & & & & 6:59:46 & & $6: 50: 25$ & & & & \\
\hline \multirow[t]{2}{*}{ Proteus } & 631 & 101 & 32 & $6 / 4 / 2003$ & OUT & TK & 805 & 7:07:20 & 52 & 7:21:49 & 40 & Y & 12.13 & 15.78 \\
\hline & & & & & & & & 7:08:12 & & 7:22:29 & & & & \\
\hline \multirow[t]{2}{*}{ Clipper Lady } & 711 & 106 & 34 & $6 / 4 / 2003$ & IN & LN & 830 & 7:28:11 & 50 & 7:15:37 & 44 & Y & 14.22 & 16.16 \\
\hline & & & & & & & & 7:29:01 & & 7:16:21 & & & & \\
\hline \multirow[t]{2}{*}{ Ionia } & 610 & 93 & 21 & $6 / 4 / 2003$ & OUT & $B C$ & 820 & 7:22:55 & 46 & $7: 36: 15$ & 35 & Y & 13.26 & 17.43 \\
\hline & & & & & & & & 7:23:41 & & 7:36:50 & & & & \\
\hline \multirow[t]{2}{*}{ Maritime Jingan } & 591 & 106 & 24 & $6 / 4 / 2003$ & In & CT & 840 & 7:40:08 & 36 & 7:28:52 & 32 & Y & 16.42 & 18.47 \\
\hline & & & & & & & & 7:40:44 & & $7: 29: 24$ & & & & \\
\hline \multirow[t]{2}{*}{ Africian Evergreen } & 445 & 62 & 14 & $6 / 4 / 2003$ & IIN & $B C$ & 845 & 7:46:50 & 19 & 7:38:46 & 19 & $N$ & 23.42 & 23.42 \\
\hline & & & & & & & & 7:47:09 & & 7:39:05 & & & & \\
\hline \multirow[t]{2}{*}{ Meriom Pearl (T) } & 559 & 95 & 36.5 & $6 / 4 / 2003$ & In & TK & 930 & 8:32:20 & 117 & 8:13:22 & 44 & $N$ & 4.78 & 12.70 \\
\hline & & & & & & & & 8:34:17 & & 8:14:06 & & & & \\
\hline \multirow[t]{2}{*}{ Hans Schulte $(T)$} & 558 & 83 & 26 & $6 / 4 / 2003$ & In & CS & 1025 & 9:29:00 & 66 & $9: 12: 57$ & 39 & $N$ & 8.45 & 14.31 \\
\hline & & & & & & & & 9:30:06 & & 9:13:36 & & & & \\
\hline \multirow[t]{2}{*}{ Corinthiakos } & 555 & 85 & 29 & $6 / 4 / 2003$ & In & BB & 1055 & 9:54:25 & 39 & 9:42:42 & 32 & $N$ & 14.23 & 17.34 \\
\hline & & & & & & & & 9:55:04 & & 9:43:14 & & & & \\
\hline \multirow[t]{2}{*}{ Panam Pacifico } & 440 & 67 & 16 & $6 / 4 / 2003$ & In & CT & 1100 & 10:00:23 & 32 & 9:49:03 & 24 & $N$ & 13.75 & 18.33 \\
\hline & & & & & & & & 10:00:55 & & $9: 49: 27$ & & & & \\
\hline \multirow[t]{2}{*}{ Sunshine Sky } & 423 & 72 & 26 & $6 / 4 / 2003$ & OUT & CT & 1125 & 10:25:18 & 26 & $10: 35: 45$ & 21 & $\mathrm{~N}$ & 16.27 & 20.14 \\
\hline & & & & & & & & $10: 25: 44$ & & 10:36:06 & & & & \\
\hline \multirow[t]{2}{*}{ Norgas Sonoma } & 410 & 65 & 20 & $6 / 4 / 2003$ & OUT & LN & 1250 & 11:49:12 & 21 & 11:58:00 & 17 & Y & 19.52 & 24.12 \\
\hline & & & & & & & & 11:49:33 & & 11:58:17 & & & & \\
\hline \multirow[t]{2}{*}{ SKS Tiete } & 799 & 138 & 40 & $6 / 4 / 2003$ & IIN & OB & 1255 & 11:57:34 & 67 & 11:44:09 & 51 & Y & 11.93 & 15.67 \\
\hline & & & & & & & & 11:58:41 & & 11:45:00 & & & & \\
\hline \multirow[t]{2}{*}{ Prodicos } & 631 & 101 & 25 & $6 / 4 / 2003$ & OUT & TK & 1320 & 12:21:04 & 44 & 12:31:52 & 33 & $N$ & 14.34 & 19.12 \\
\hline & & & & & & & & 12:21:48 & & $12: 32: 25$ & & & & \\
\hline
\end{tabular}




\begin{tabular}{|c|c|c|c|c|c|c|c|c|c|c|c|c|c|c|}
\hline Ship Name & $\begin{array}{l}\text { Length } \\
\mathrm{ft}\end{array}$ & $\begin{array}{l}\text { Beam } \\
\mathrm{ft}\end{array}$ & $\begin{array}{l}\text { Draft } \\
\mathrm{ft}\end{array}$ & Date & Direction & $\begin{array}{l}\text { Ship } \\
\text { Type }\end{array}$ & $\begin{array}{l}\text { Time- } \\
\text { MP log }\end{array}$ & $\begin{array}{l}\text { Time** } \\
\text { at NC }\end{array}$ & $\begin{array}{l}\text { B-S } \\
\text { at NC } \\
\text { sec }\end{array}$ & $\begin{array}{l}\text { Time** } \\
\text { at SC }\end{array}$ & $\begin{array}{l}\text { B-S } \\
\text { at SC } \\
\text { sec }\end{array}$ & $\begin{array}{l}\text { Meeting } \\
\text { Ships }\end{array}$ & $\begin{array}{l}\text { NC } \\
\text { Speed } \\
\text { fps }\end{array}$ & $\begin{array}{l}\text { SC } \\
\text { Speed } \\
\text { fps }\end{array}$ \\
\hline \multirow[t]{2}{*}{$\begin{array}{l}\text { Lykes } \\
\text { Ambassador (T) }\end{array}$} & 889 & 106 & 32 & $6 / 4 / 2003$ & OUT & CS & 1335 & 12:34:41 & 105 & $12: 47: 42$ & 47 & $N$ & 8.47 & 18.91 \\
\hline & & & & & & & & 12:36:26 & & 12:48:29 & & & & \\
\hline \multirow[t]{2}{*}{ Clipper Sea } & 458 & 67 & 27 & $6 / 4 / 2003$ & IN & LN & 1405 & 13:09:07 & 24 & 13:00:12 & 21 & $N$ & 19.08 & 21.81 \\
\hline & & & & & & & & 13:09:31 & & 13:00:33 & & & & \\
\hline \multirow[t]{2}{*}{ Agrari } & 751 & 106 & 29 & $6 / 4 / 2003$ & IN & TK & 1430 & 13:32:11 & 47 & 13:21:37 & 39 & $N$ & 15.98 & 19.26 \\
\hline & & & & & & & & 13:32:58 & & 13:22:16 & & & & \\
\hline \multirow[t]{2}{*}{ United Reliance } & 830 & 146 & 34 & $6 / 4 / 2003$ & IN & TK & 1530 & 14:31:12 & 74 & $14: 17: 31$ & 51 & $N$ & 11.22 & 16.27 \\
\hline & & & & & & & & 14:32:26 & & 14:18:22 & & & & \\
\hline \multirow[t]{2}{*}{ Lochness } & 810 & 136 & 40 & $6 / 4 / 2003$ & IN & TK & 1615 & 15:16:22 & 80 & 15:01:27 & 56 & $N$ & 10.13 & 14.46 \\
\hline & & & & & & & & 15:17:42 & & $15: 02: 23$ & & & & \\
\hline \multirow[t]{2}{*}{ Lofoten $(T)$} & 810 & 138 & 38 & $6 / 4 / 2003$ & IN & TK & 1635 & 15:37:19 & 72 & $15: 22: 40$ & 56 & $\mathrm{~N}$ & 11.25 & 14.46 \\
\hline & & & & & & & & 15:38:31 & & 15:23:36 & & & & \\
\hline \multirow[t]{2}{*}{ Rhine Trader } & 328 & 56 & 21 & $6 / 4 / 2003$ & OUT & BB & 1640 & $15: 37: 48$ & 16 & $15: 46: 33$ & 14 & $\mathrm{~N}$ & 20.50 & 23.43 \\
\hline & & & & & & & & 15:38:04 & & $15: 46: 47$ & & & & \\
\hline \multirow[t]{2}{*}{ Berge Kobe } & 721 & 126 & 35 & $6 / 4 / 2003$ & IN & LN & 1735 & $16: 34: 23$ & 59 & $16: 22: 22$ & 42 & $N$ & 12.22 & 17.17 \\
\hline & & & & & & & & 16:35:22 & & 16:23:04 & & & & \\
\hline \multirow[t]{2}{*}{ HNI Brenton Reef } & 620 & 106 & 27 & $6 / 4 / 2003$ & IN & CT & 1755 & 16:53:14 & 50 & $16: 41: 46$ & 30 & $N$ & 12.40 & 20.67 \\
\hline & & & & & & & & 16:54:04 & & $16: 42: 16$ & & & & \\
\hline \multirow[t]{2}{*}{ MSC Zrin } & 661 & 107 & 28 & $6 / 4 / 2003$ & OUT & CS & 1805 & 17:08:07 & 78 & $17: 24: 24$ & 53 & Y & 8.47 & 12.47 \\
\hline & & & & & & & & 17:09:25 & & $17: 25: 17$ & & & & \\
\hline \multirow[t]{2}{*}{ Domenico levoli } & 479 & 71 & 23 & $6 / 4 / 2003$ & IN & CT & 1825 & 17:26:50 & 38 & $17: 14: 50$ & 27 & Y & 12.61 & 17.74 \\
\hline & & & & & & & & 17:27:28 & & $17: 15: 17$ & & & & \\
\hline \multirow[t]{2}{*}{ Pacific Sapphire } & 810 & 137 & 36 & $6 / 4 / 2003$ & IN & TK & 1840 & 17:41:39 & 69 & 17:28:06 & 56 & $N$ & 11.74 & 14.46 \\
\hline & & & & & & & & $17: 42: 48$ & & 17:29:02 & & & & \\
\hline \multirow[t]{2}{*}{ UAL Africa } & 424 & 56 & 16 & $6 / 4 / 2003$ & IN & BB & 1850 & 17:52:15 & 32 & 17:38:37 & 27 & $N$ & 13.25 & 15.70 \\
\hline & & & & & & & & 17:52:47 & & 17:39:04 & & & & \\
\hline \multirow[t]{2}{*}{ Ioannis } & 599 & 106 & 26 & $6 / 4 / 2003$ & IN & TK & 1910 & 18:09:27 & 43 & 17:58:00 & 30 & $\mathrm{~N}$ & 13.93 & 19.97 \\
\hline & & & & & & & & 18:10:10 & & 17:58:30 & & & & \\
\hline \multirow[t]{2}{*}{ Erikousa } & 748 & 106 & 27 & $6 / 4 / 2003$ & OUT & TK & 1940 & 18:41:25 & 51 & 18:51:37 & 36 & $N$ & 14.67 & 20.78 \\
\hline & & & & & & & & 18:42:16 & & $18: 52: 13$ & & & & \\
\hline \multirow[t]{2}{*}{ Rickmers Tokyo } & 633 & 91 & 30 & $6 / 4 / 2003$ & IN & BB & 2100 & 19:59:55 & 47 & 19:49:17 & 29 & $N$ & 13.47 & 21.83 \\
\hline & & & & & & & & 20:00:42 & & 19:49:46 & & & & \\
\hline \multirow[t]{2}{*}{ Jo Sypress } & 598 & 105 & 25 & $6 / 4 / 2003$ & OUT & CT & 2335 & 22:33:37 & 33 & $22: 43: 11$ & 29 & $N$ & 18.12 & 20.62 \\
\hline & & & & & & & & $22: 34: 10$ & & $22: 43: 40$ & & & & \\
\hline \multirow[t]{2}{*}{ Igloo Moon } & 465 & 72 & 26 & $6 / 5 / 2003$ & OUT & LN & 240 & 1:37:45 & 28 & 1:47:25 & 20 & $N$ & 16.61 & 23.25 \\
\hline & & & & & & & & 1:38:13 & & 1:47:45 & & & & \\
\hline
\end{tabular}




\begin{tabular}{|c|c|c|c|c|c|c|c|c|c|c|c|c|c|c|}
\hline Ship Name & $\begin{array}{l}\text { Length } \\
\mathrm{ft}\end{array}$ & $\begin{array}{l}\text { Beam } \\
\mathrm{ft}\end{array}$ & $\begin{array}{l}\text { Draft } \\
\mathrm{ft}\end{array}$ & Date & Direction & $\begin{array}{l}\text { Ship } \\
\text { Type }\end{array}$ & $\begin{array}{l}\text { Time- } \\
\text { MP log }\end{array}$ & $\begin{array}{l}\text { Time** } \\
\text { at NC }\end{array}$ & \begin{tabular}{|l} 
B-S \\
at NC \\
sec
\end{tabular} & $\begin{array}{l}\text { Time** } \\
\text { at SC }\end{array}$ & \begin{tabular}{|l} 
B-S \\
at SC \\
sec
\end{tabular} & $\begin{array}{l}\text { Meeting } \\
\text { Ships }\end{array}$ & \begin{tabular}{|l|} 
NC \\
Speed \\
fps
\end{tabular} & \begin{tabular}{|l|} 
SC \\
Speed \\
fps
\end{tabular} \\
\hline \multirow[t]{2}{*}{$\begin{array}{l}\text { Dimitrovskiy } \\
\text { Komsomol }\end{array}$} & 651 & 91 & 36 & $6 / 5 / 2003$ & OUT & $B C$ & 350 & 2:50:00 & 43 & 3:02:57 & 40 & $\mathrm{Y}$ & 15.14 & 16.28 \\
\hline & & & & & & & & 2:50:43 & & 3:03:37 & & & & \\
\hline \multirow[t]{2}{*}{ Stolt Topaz } & 580 & 106 & 31 & 6/5/2003 & & CT & 355 & 2:58:23 & 45 & 2:45:45 & 31 & $Y$ & 12.89 & 18.71 \\
\hline & & & & & & & & 2:59:08 & & 2:46:16 & & & & \\
\hline \multirow[t]{2}{*}{ Apman II } & 518 & 75 & 21 & 6/5/2003 & & BB & 530 & 4:29:00 & 30 & 4:18:44 & 26 & $\mathrm{~N}$ & 17.27 & 19.92 \\
\hline & & & & & & & & 4:29:30 & & 4:19:10 & & & & \\
\hline \multirow[t]{2}{*}{ Karonga } & 566 & 76 & 26 & $6 / 5 / 2003$ & OUT & BB & 545 & 4:47:59 & 41 & 4:58:59 & 30 & $\mathrm{~N}$ & 13.80 & 18.87 \\
\hline & & & & & & & & 4:48:40 & & 4:59:29 & & & & \\
\hline \multirow[t]{2}{*}{ Panam Atlantico } & 442 & 68 & 24 & $6 / 5 / 2003$ & OUT & CT & 700 & 6:01:44 & 36 & 6:11:45 & 21 & $\mathrm{~N}$ & 12.28 & 21.05 \\
\hline & & & & & & & & 6:02:10 & & 6:12:06 & & & & \\
\hline \multirow[t]{2}{*}{ SKS Trent } & 800 & 138 & 30 & $6 / 5 / 2003$ & OUT & OB & 715 & 6:15:48 & 57 & 6:26:47 & 42 & $\mathrm{~N}$ & 14.04 & 19.05 \\
\hline & & & & & & & & 6:16:45 & & 6:27:29 & & & & \\
\hline \multirow[t]{2}{*}{ Gaz Progress } & 771 & 118 & 26 & $6 / 5 / 2003$ & OUT & LN & 725 & 6:26:10 & 57 & 6:37:32 & 41 & $\mathrm{~N}$ & 13.53 & 18.80 \\
\hline & & & & & & & & 6:27:07 & & 6:38:13 & & & & \\
\hline \multirow[t]{2}{*}{ Sanko Quality } & 810 & 138 & 28 & $6 / 5 / 2003$ & OUT & TK & 750 & 6:50:55 & 73 & 7:04:05 & 50 & $\mathrm{~N}$ & 11.10 & 16.20 \\
\hline & & & & & & & & 6:52:08 & & 7:04:55 & & & & \\
\hline \multicolumn{15}{|c|}{ (Sheet 6 of 6 , } \\
\hline \multicolumn{15}{|c|}{$\begin{array}{l}\text { Note: Times are central standard time, cameras record in CST, Morgans Point Log time is converted from CDT. } \\
\text { * NC time corrected was not in sync with GPS time and had to be corrected by adding } 2 \text { minutes } 16 \text { seconds. } \\
\text { ** Top entry is time at camera when bow of ship is in center of field of view, bottom entry is stern time. } \\
\text { B = bow, } \mathrm{S}=\text { stern. } \\
\text { MP = Morgans Point, NC = north camera, SC = south camera. } \\
\text { (T) = Tug assist observed at North camera. Tugs could not be seen at nighttime or if on the side of ship opposite to camera. } \\
\text { CT = Chemical Tanker, CS = Container Ship, BB = Break Bulk Carrier, TK = Tanker, LN = Gas Carrier, BC = Bulk Carrier. } \\
\text { OB = Ore Boat, } \mathrm{CP}=\text { Container Parcel, } \mathrm{VC}=\text { Vehicle Carrier. }\end{array}$} \\
\hline
\end{tabular}

Table 3. Selected ships for data presentation.

\begin{tabular}{|l|l|l|l|l|l|}
\hline Ship & Direction & $\begin{array}{l}\text { Date/time at Morgan's } \\
\text { Point, CST }\end{array}$ & Type* & $\begin{array}{l}\text { Maximum } \\
\text { Drawdown, } \mathrm{ft}^{* *}\end{array}$ & Figure Numbers \\
\hline SKS Trent & In & $6 / 3-1440$ & OB & 2.3 & $2 \mathrm{a}$ \\
\hline Agrari & In & $6 / 4-1332$ & TK & 1.2 & $2 \mathrm{~b}$ \\
\hline United Reliance & In & $6 / 4-1430$ & TK & 1.6 & $2 \mathrm{c}$ \\
\hline Berge Kobe & In & $6 / 4-1634$ & LN & 2.1 & $2 \mathrm{~d}, 3$ \\
\hline TMM Hermosilla & Out & $6 / 3-0217$ & CS & 1.7 & $5 \mathrm{e}$ \\
\hline Eagle Suburu & Out & $6 / 3-1549$ & TK & 2.0 & $5 \mathrm{f}$ \\
\hline Lykes Ambassador & Out & $6 / 4-1235$ & CS & 1.8 & $5 \mathrm{~g}$ \\
\hline SKS Trent & Out & $6 / 5-0616$ & OB & 2.7 & $5 \mathrm{~h}$ \\
\hline Gaz Progress & Out & $6 / 5-0626$ & LN & 2.0 & $5 \mathrm{i}$ \\
\hline $\begin{array}{l}* \\
* * \text { OB }=\text { ore boat, TK }=\text { tanker, LN }=\text { gas carrier, CS }=\text { container ship. } \\
\text { ambient conditions before the ship arrives. }\end{array}$
\end{tabular}




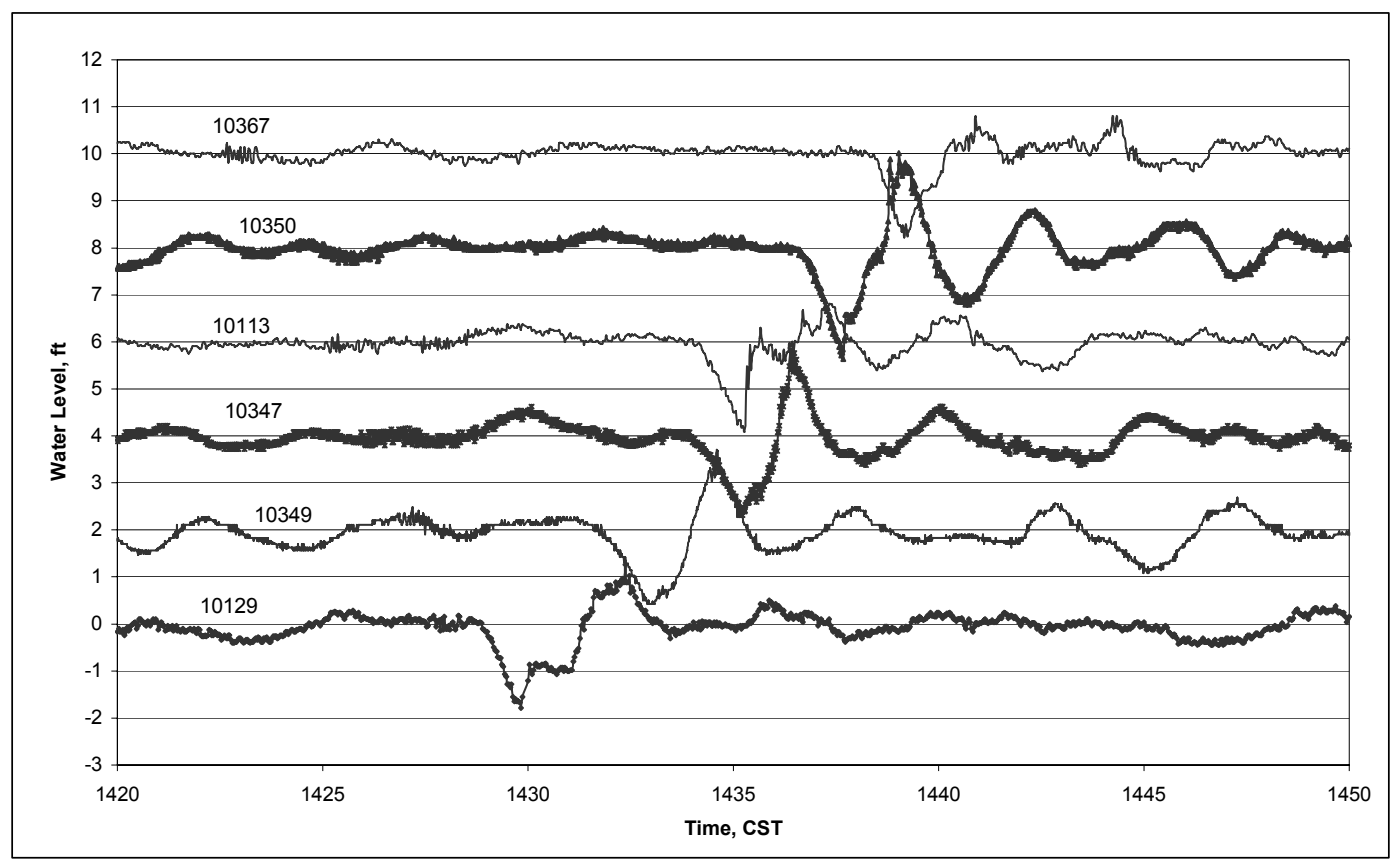

a. SKS Trent, 3 June 2003.

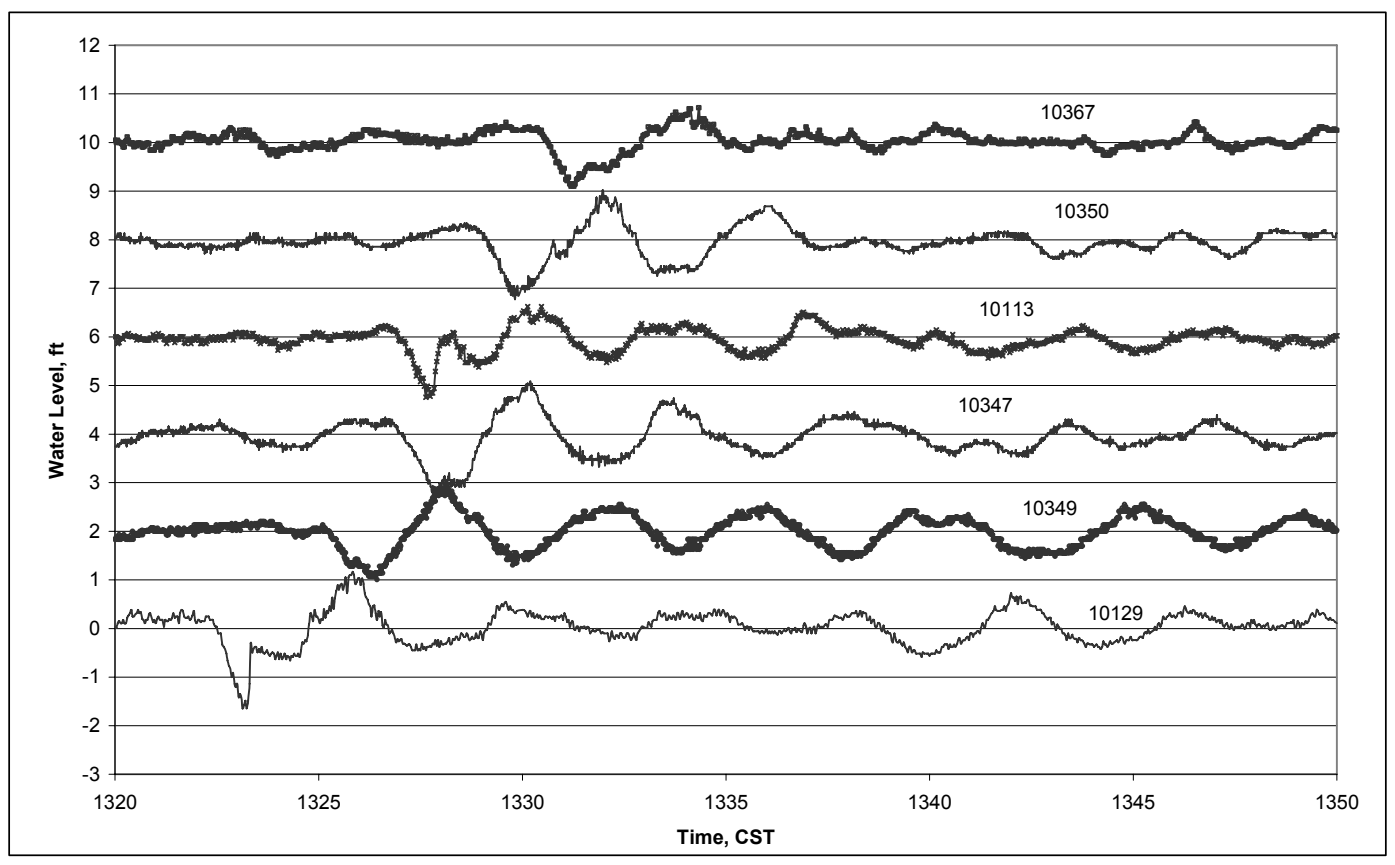

b. Agrari, 4 June 2003.

Figure 2. Time histories at all water gages during inbound ship passages (Continued). 


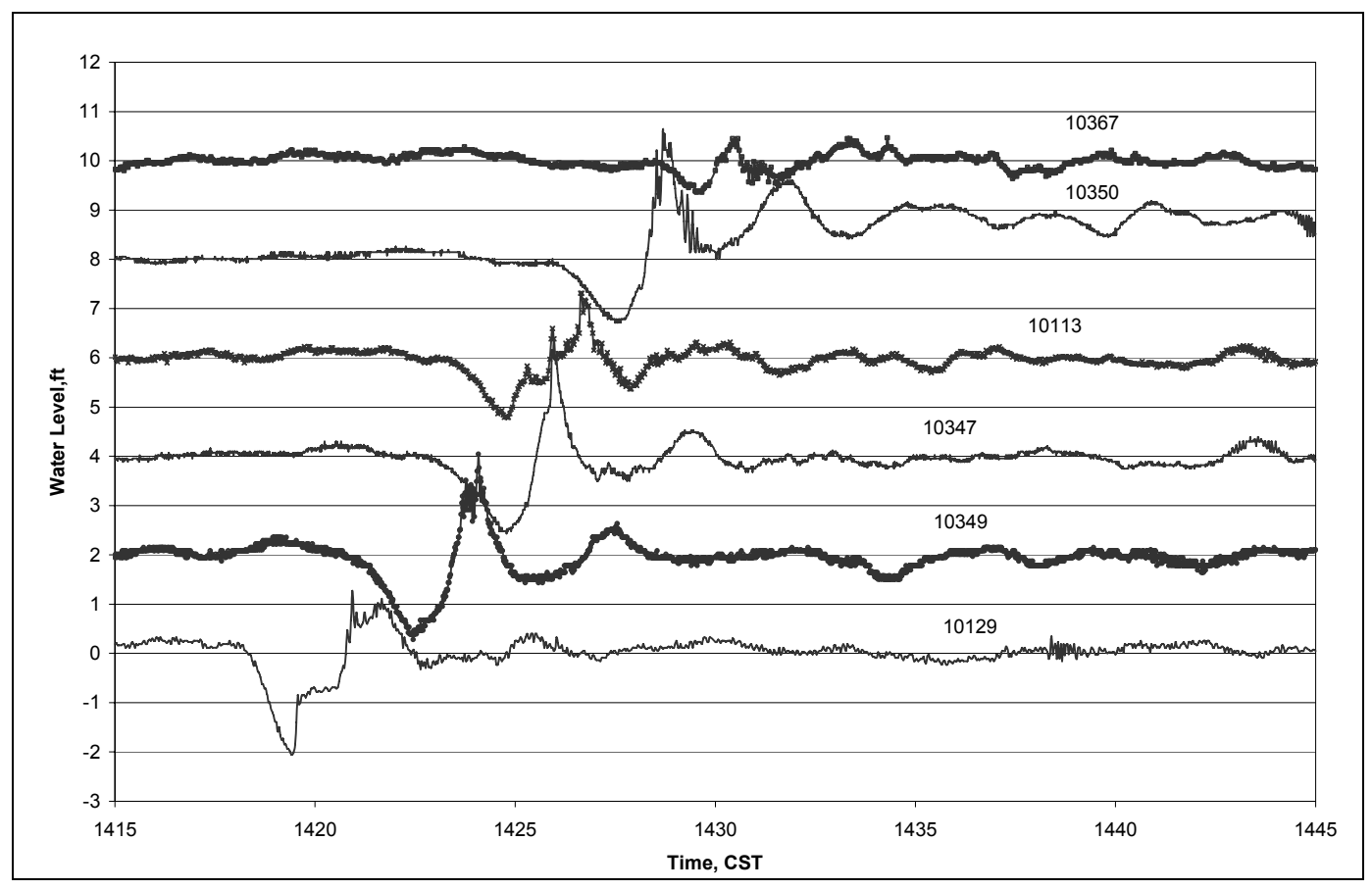

c. United Reliance, 4 June 2003.

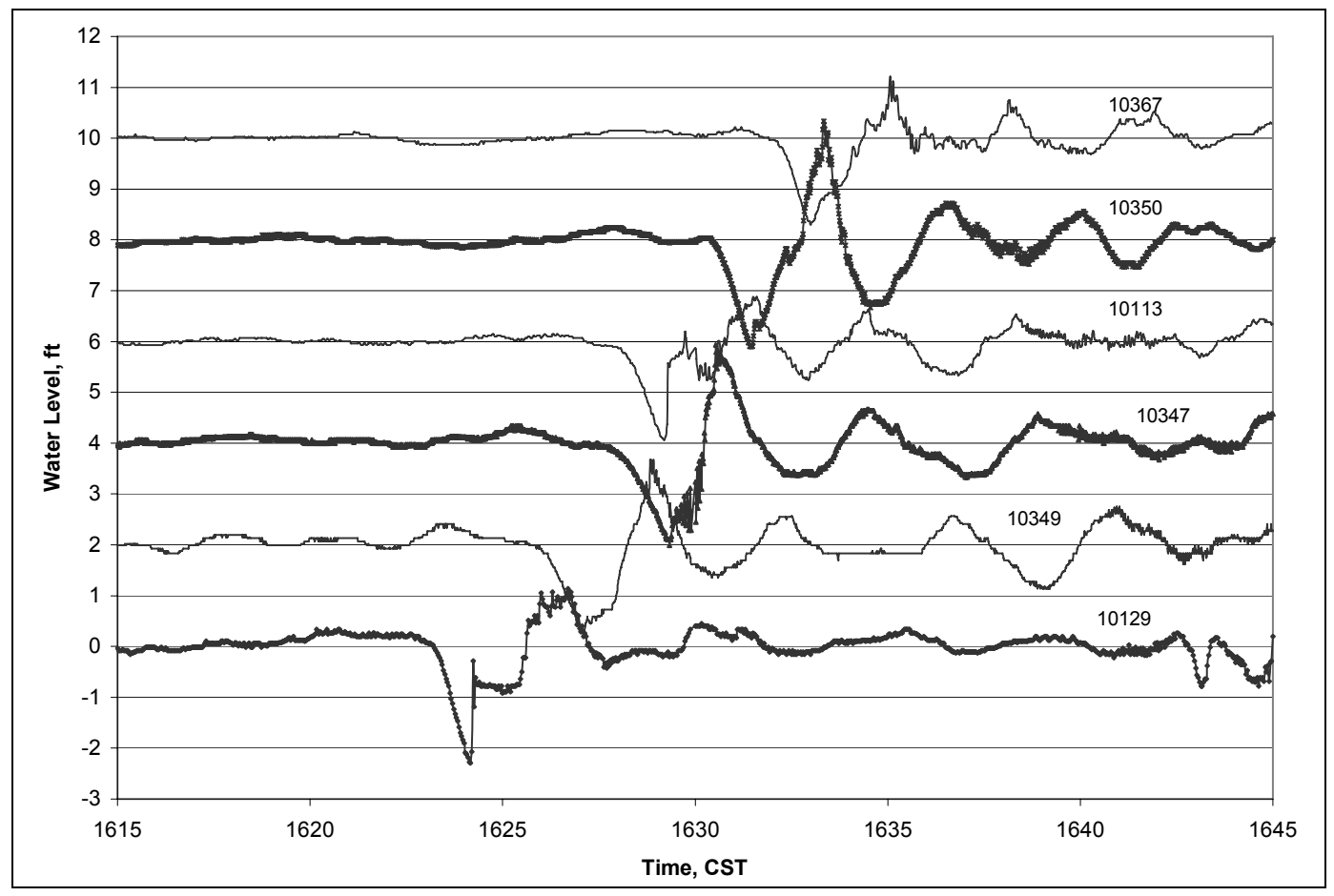

d. Berge Kobe, 4 June 2003.

Figure 2. (Concluded). 


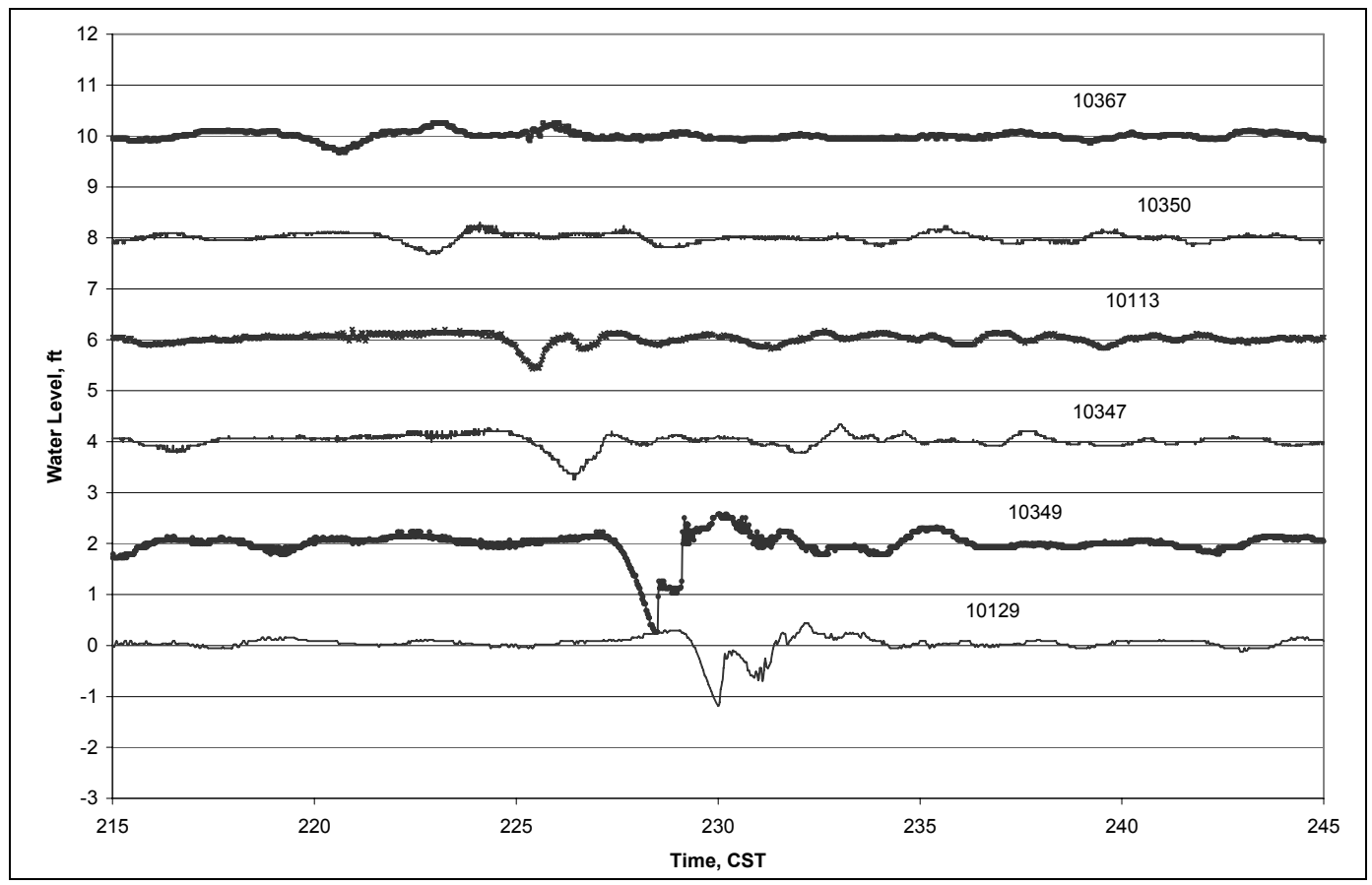

a. TMM Hermosilla, 3 June 2003.

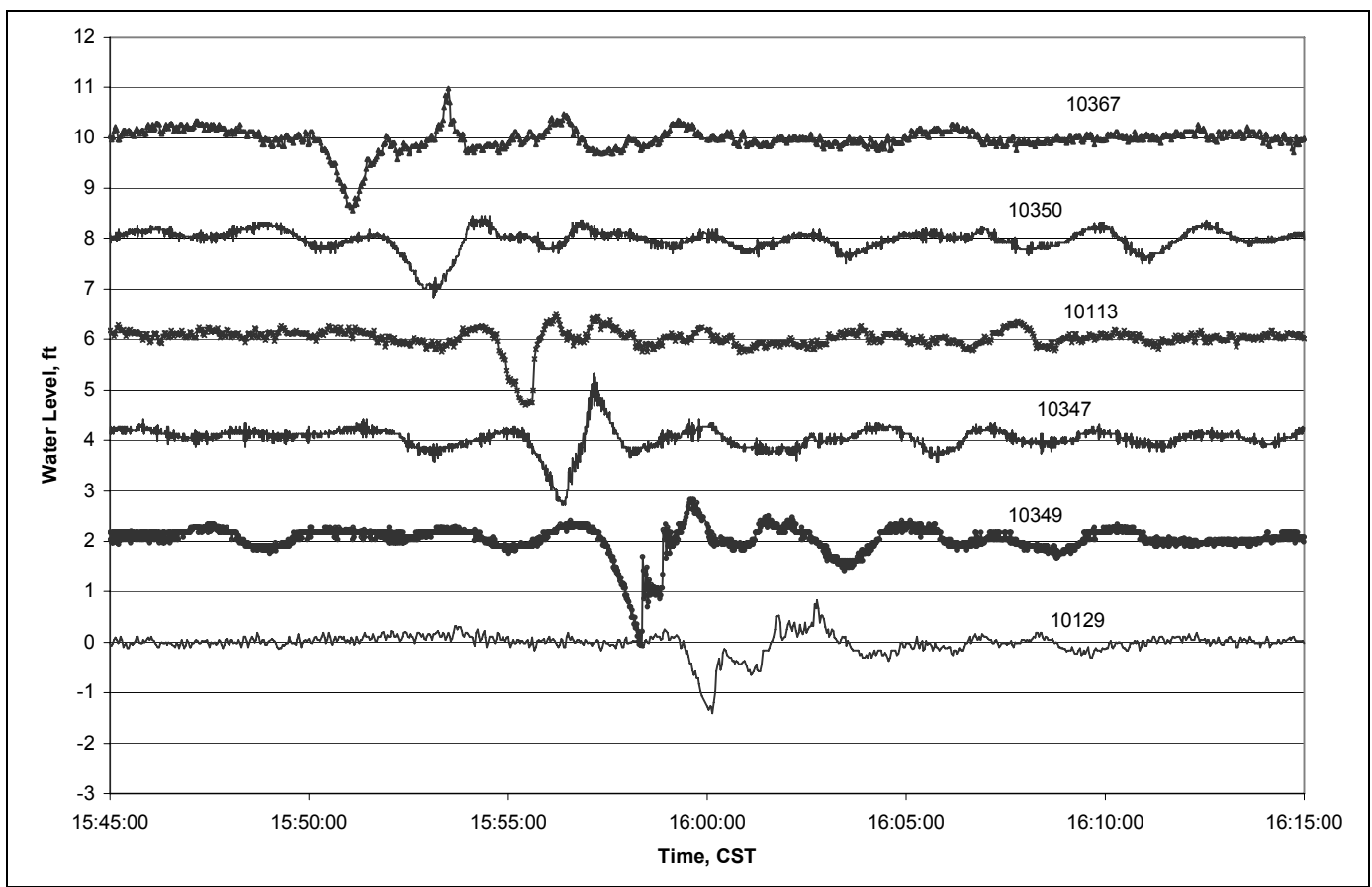

b. Eagle Subaru, 3 June 2003.

Figure 3. Time histories of all gages during outbound ship passages (Continued). 


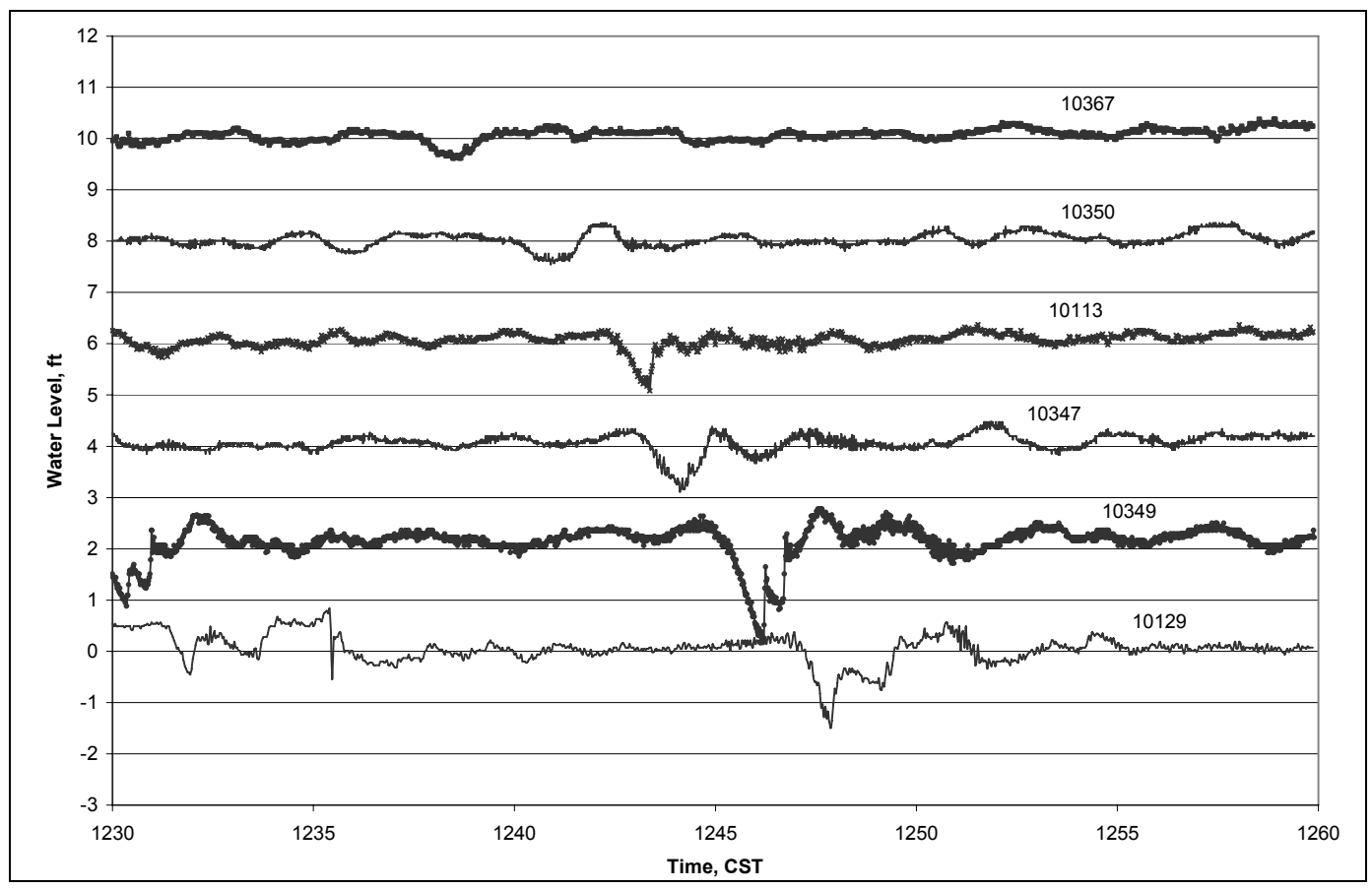

c. Lykes Ambassador, 4 June 2003.

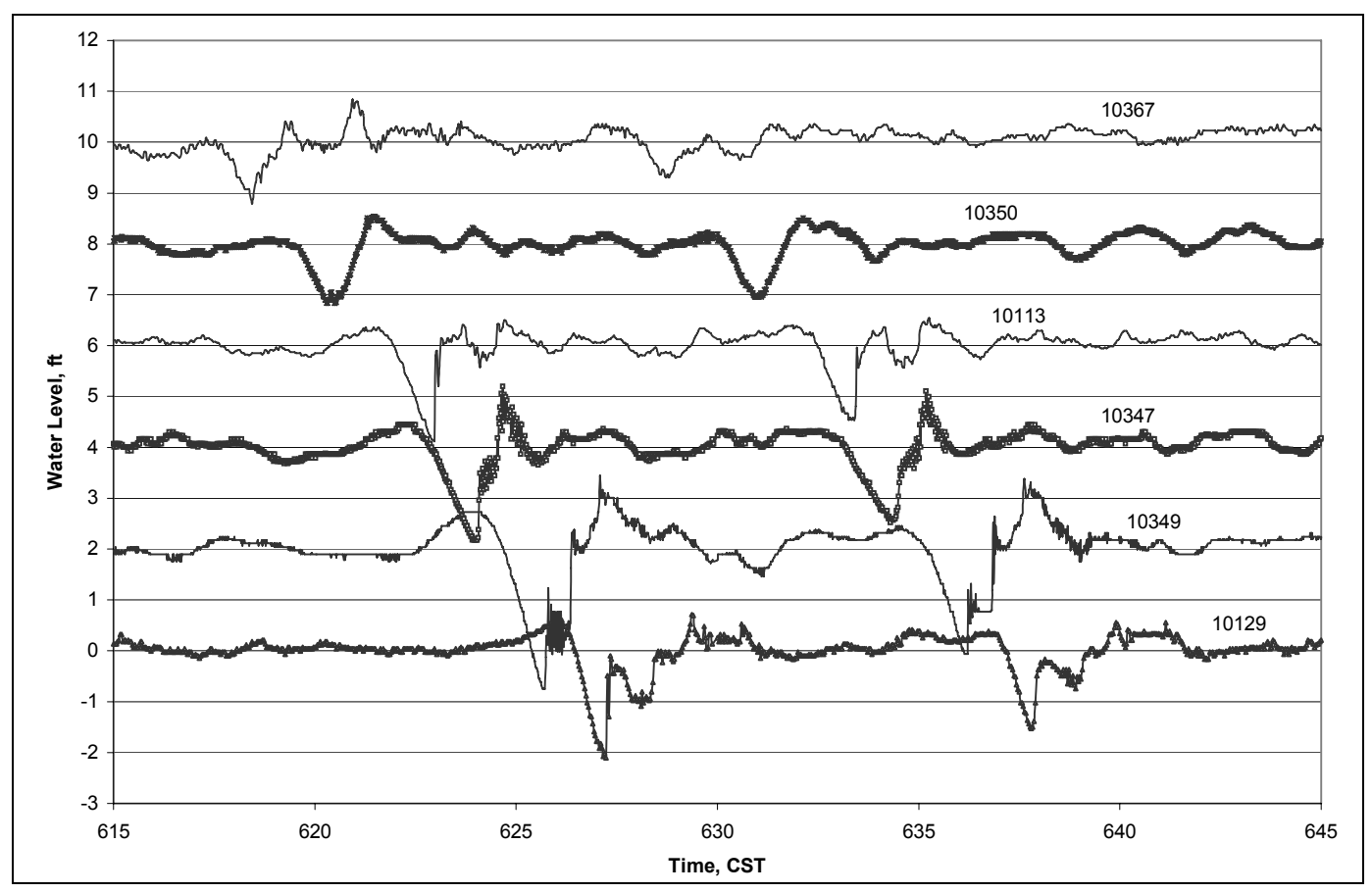

d. SKS Trent and Gaz Progress, 3 June 2003.

Figure 3. (Concluded). 


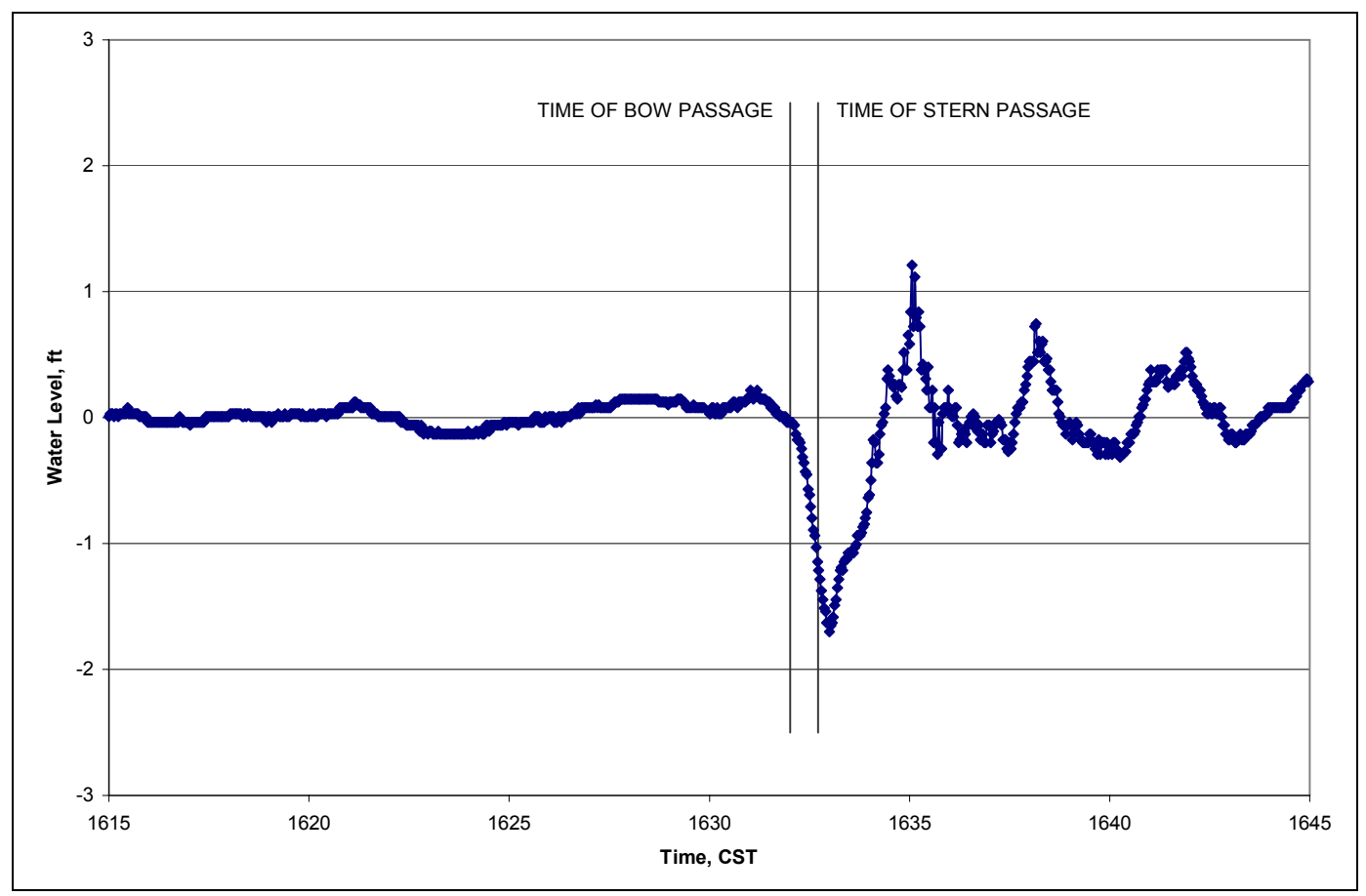

a. North navigation channel gage 10367 .

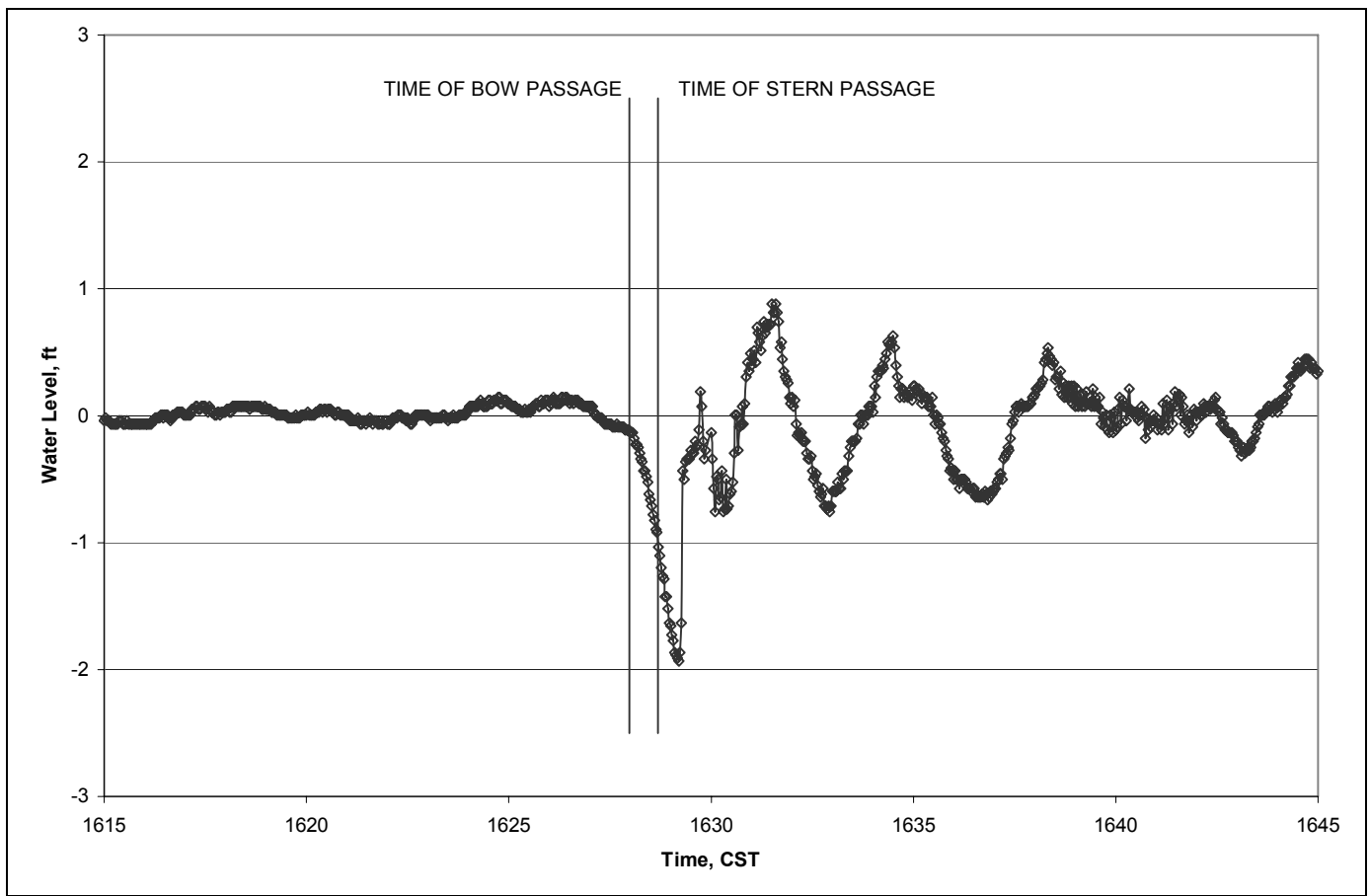

b. Middle navigation channel gage 10113 .

Figure 4. Berge Kobe, 4 June 2003 passage (Continued). 


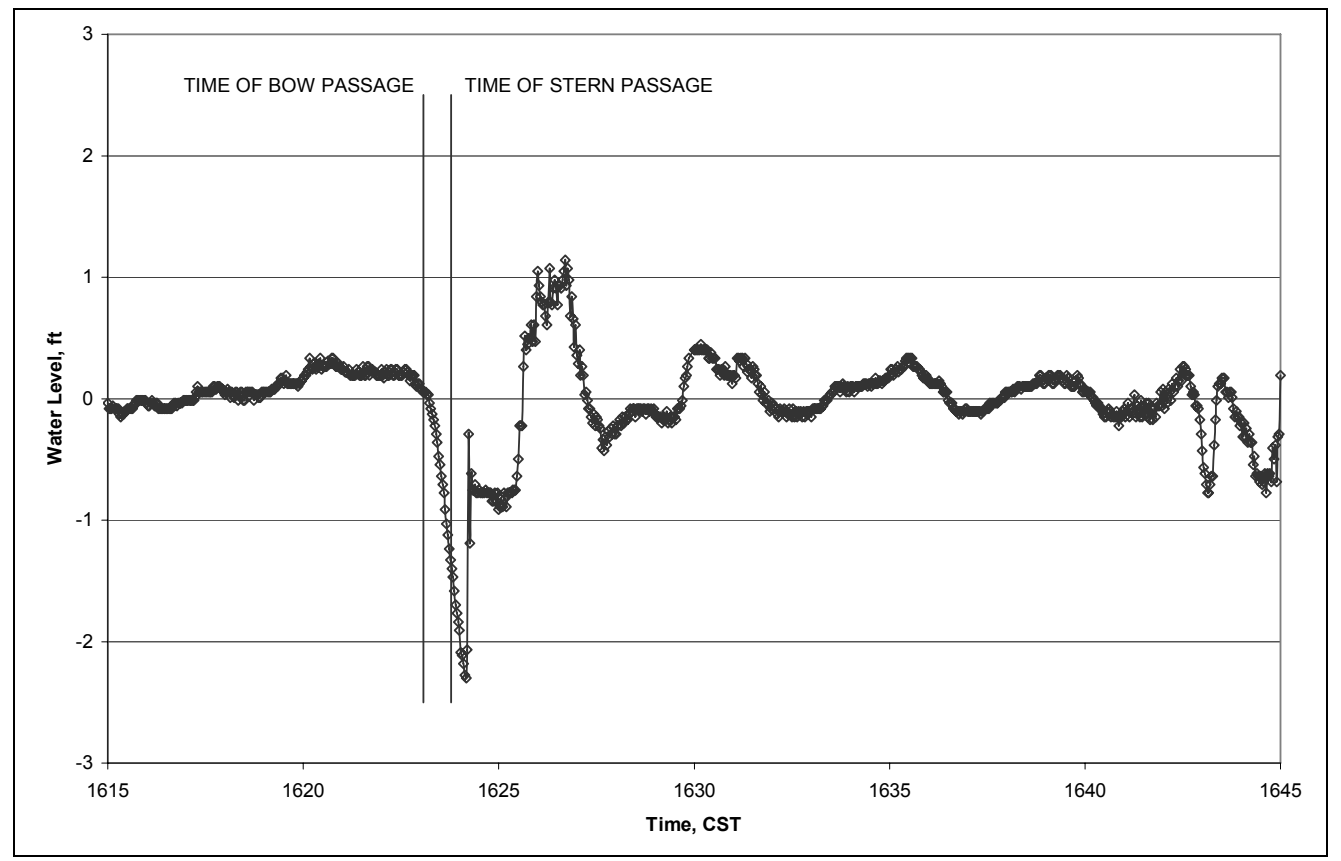

c. South navigation channel gage 10129 .

Figure 4. (Concluded).

By use of the time of arrival of the maximum drawdown at the HSC pressure gages, depth at the ship and the gage, and the gage position along and away from the HSC, average ship speed between the gages was determined. By use of the average pressure cell ship speeds and the point ship speeds from the cameras shown in Table 2, the ship speeds through the reach was determined and are shown in Figure 5. Also shown is the adopted speed curve that will be used in the numerical modeling of that ship. The speed curves show that ships were accelerating at a rate of about 0.015 knots/second for the container ship Lykes Ambassador and $0.012 \mathrm{knots} / \mathrm{second}$ for the ore boat SKS Trent.

The data collected in the field study and presented above were used to validate the numerical model of the existing channel. 


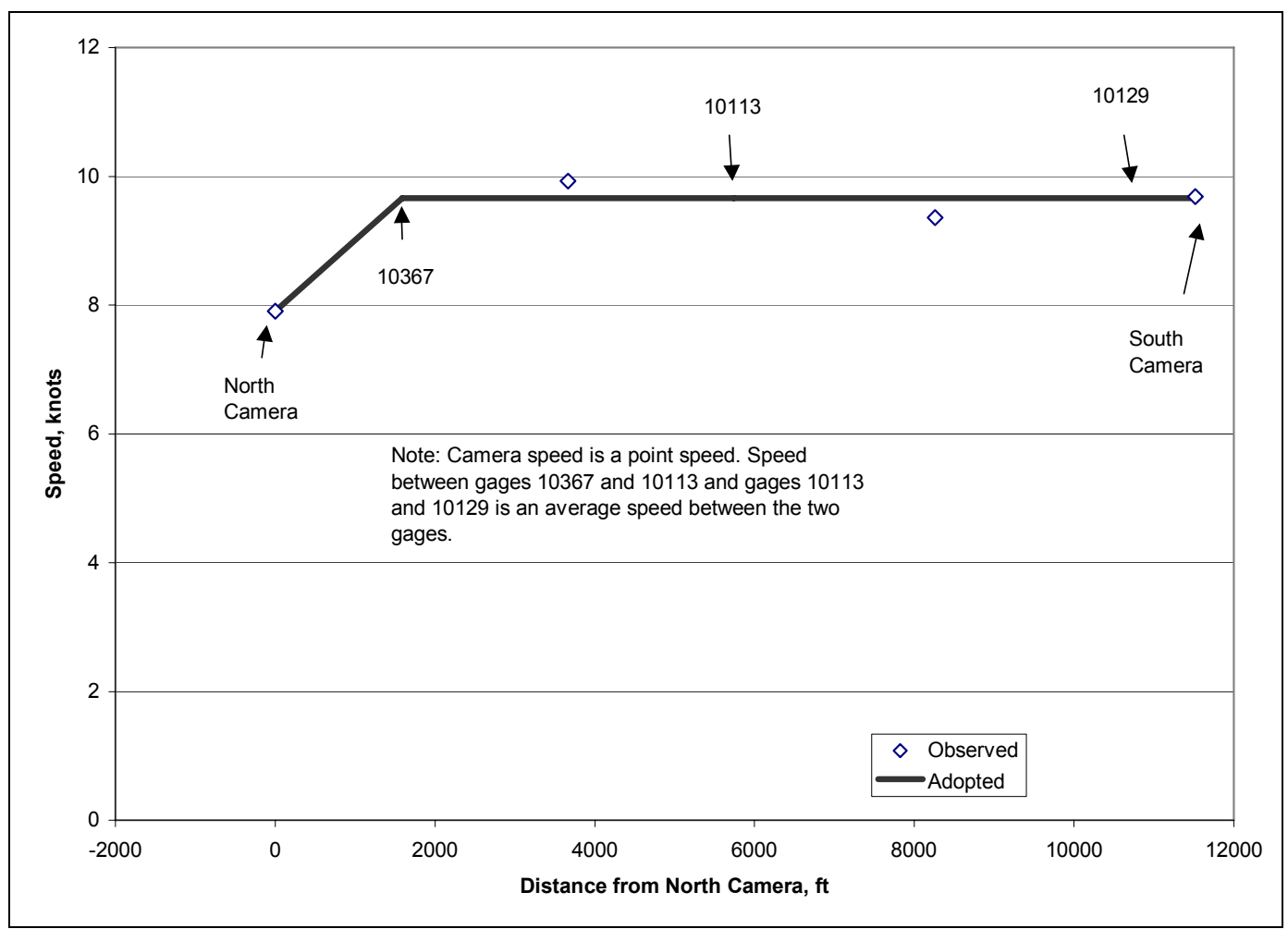

a. SKS Trent, 4 June 2003.

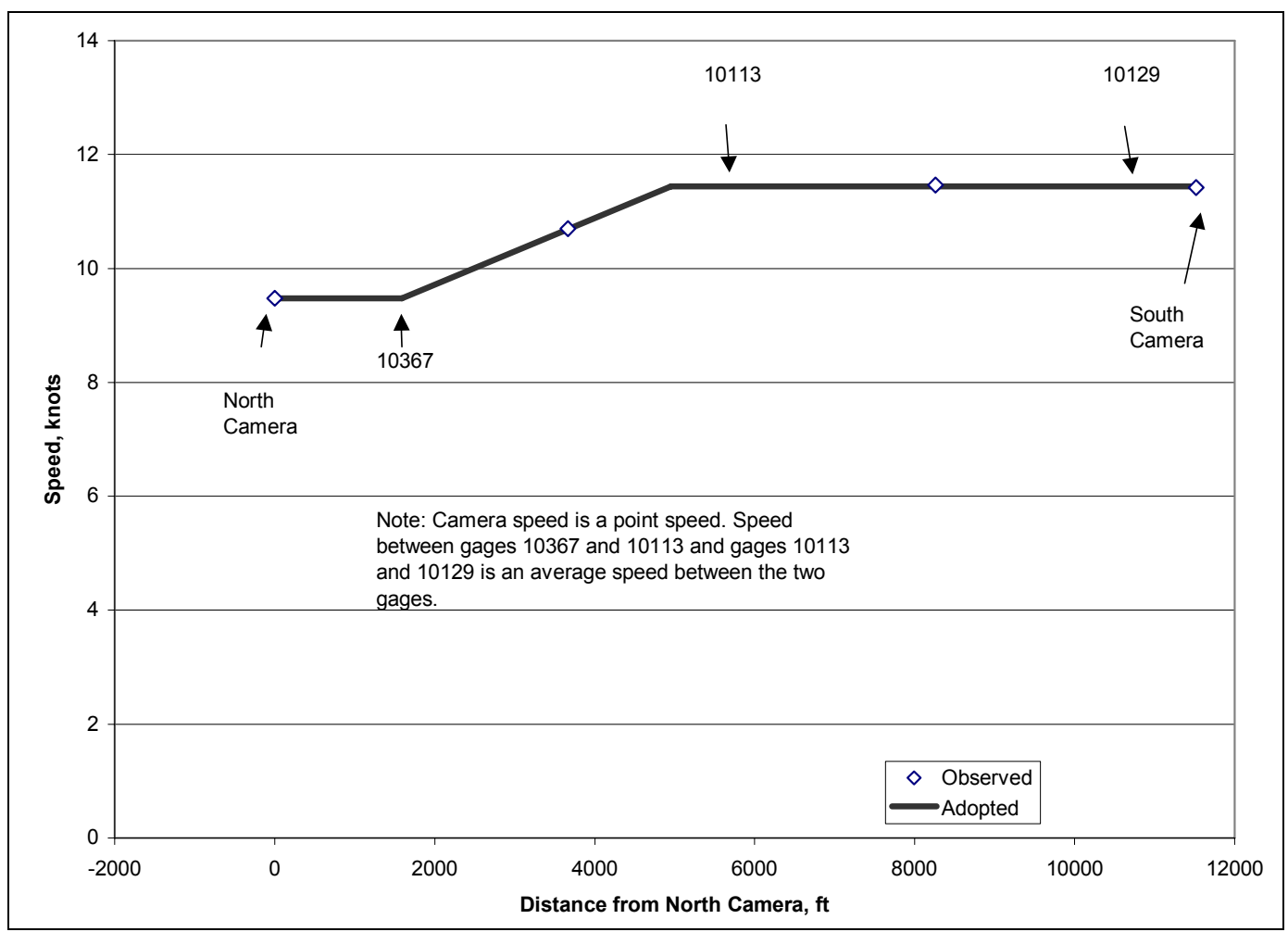

b. Agrari, inbound, 4 June 2003.

Figure 5. Ship speed along HSC (Sheet 1 of 5). 


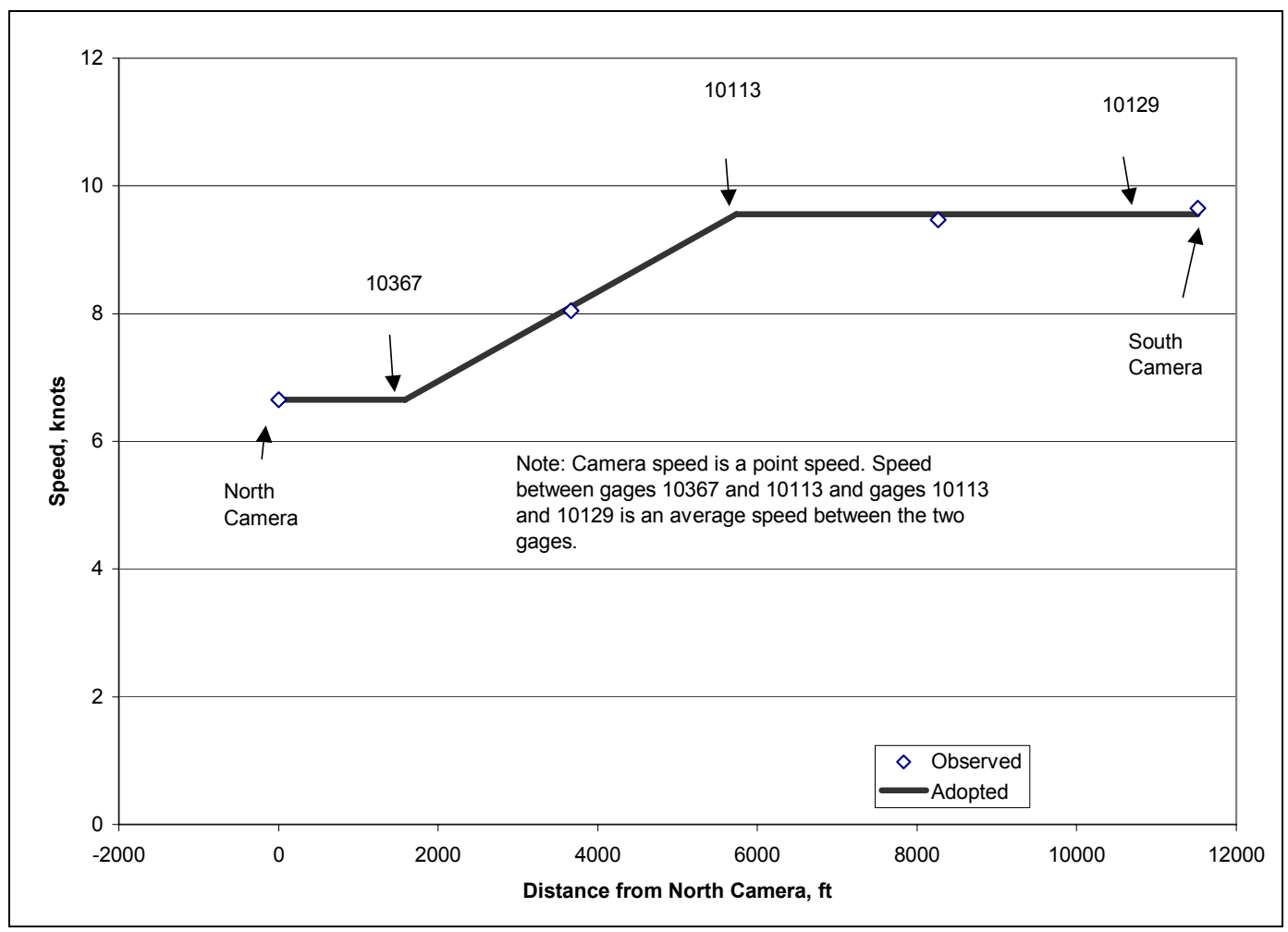

c. United Reliance, inbound, 4 June 2003.

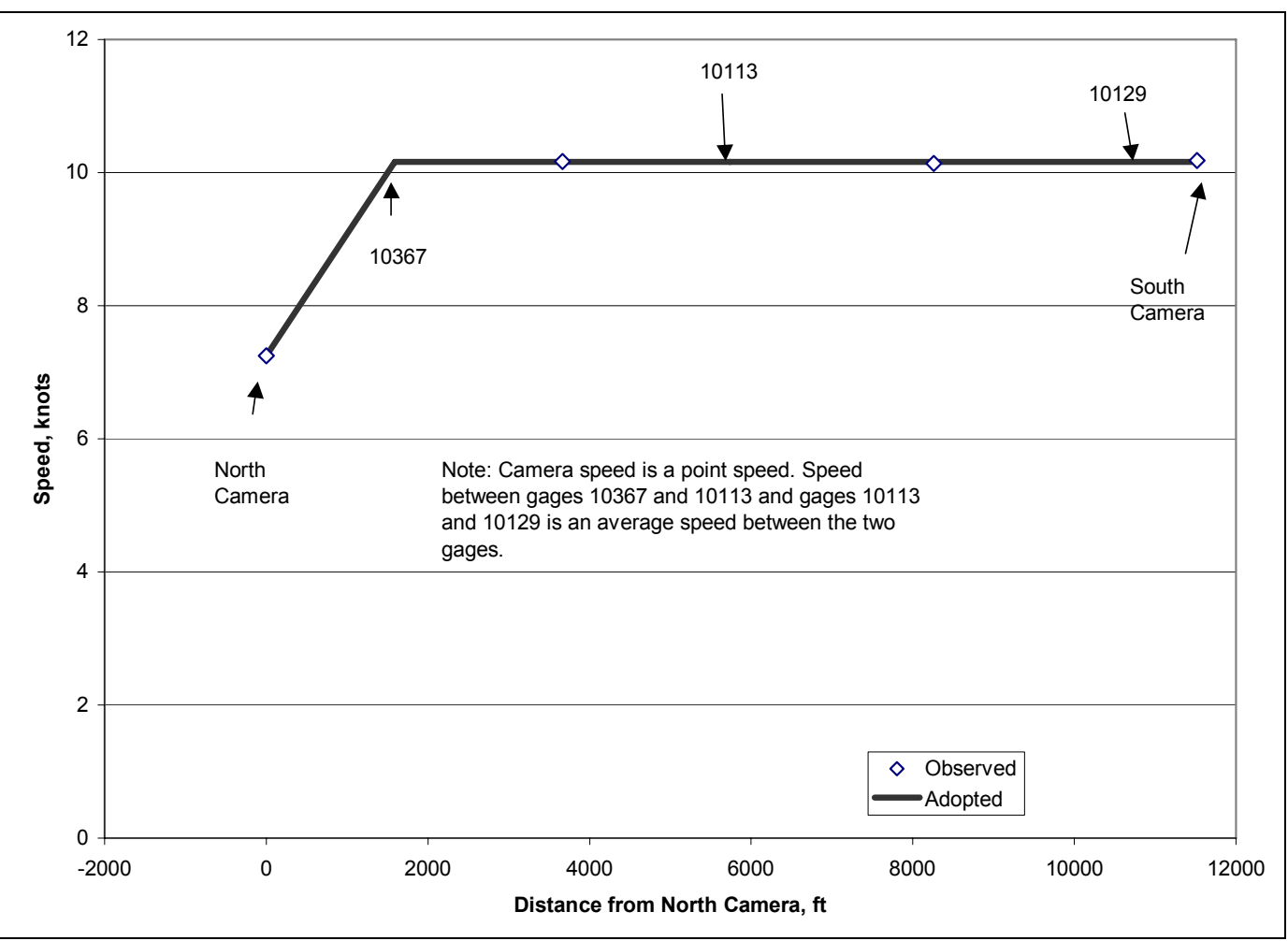

d. Berge Kobe, inbound, 4 June 2003.

Figure 5. (Sheet 2 of 5). 


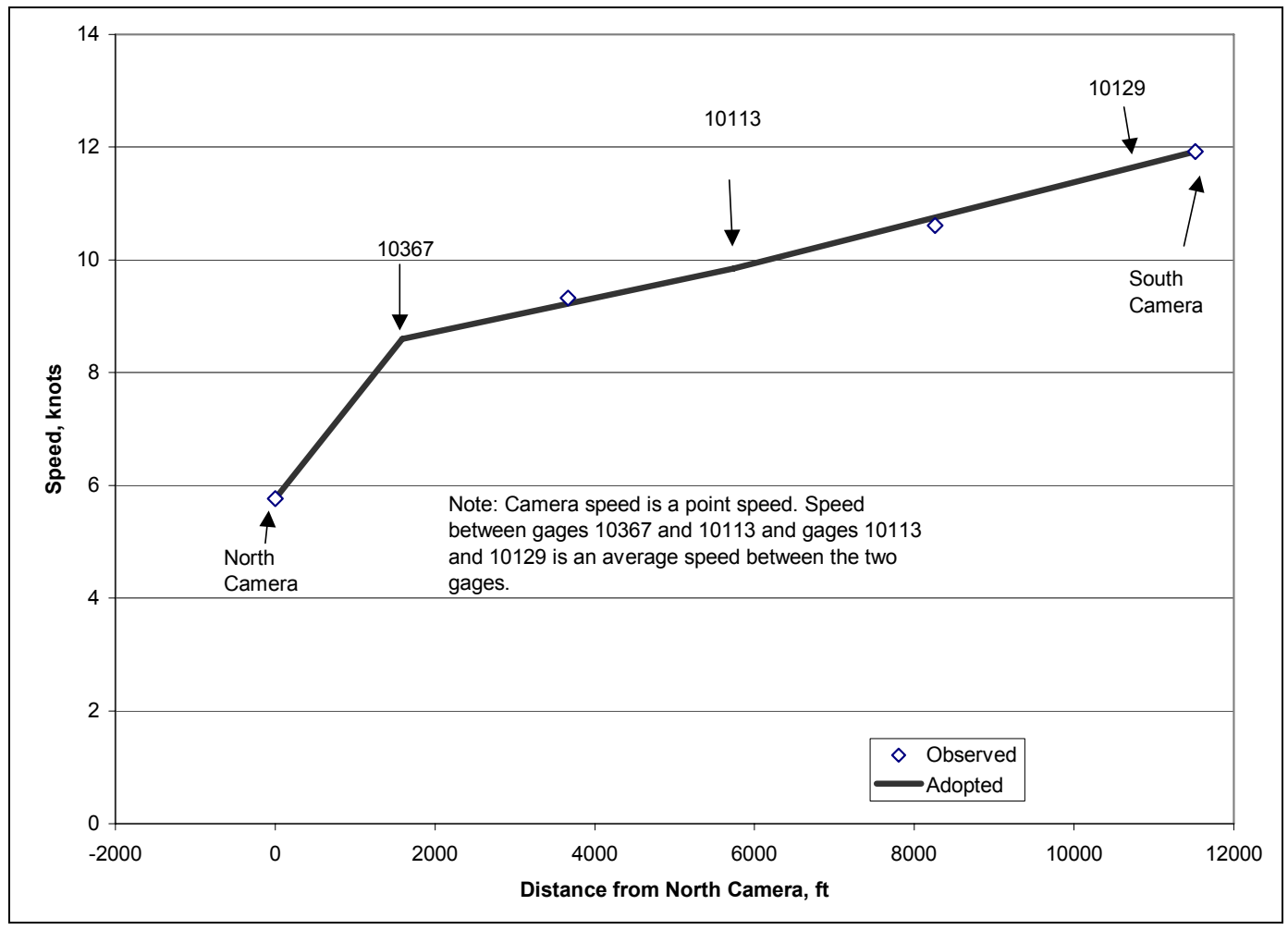

e. TMM Hermosilla, outbound, 3 June 2003.

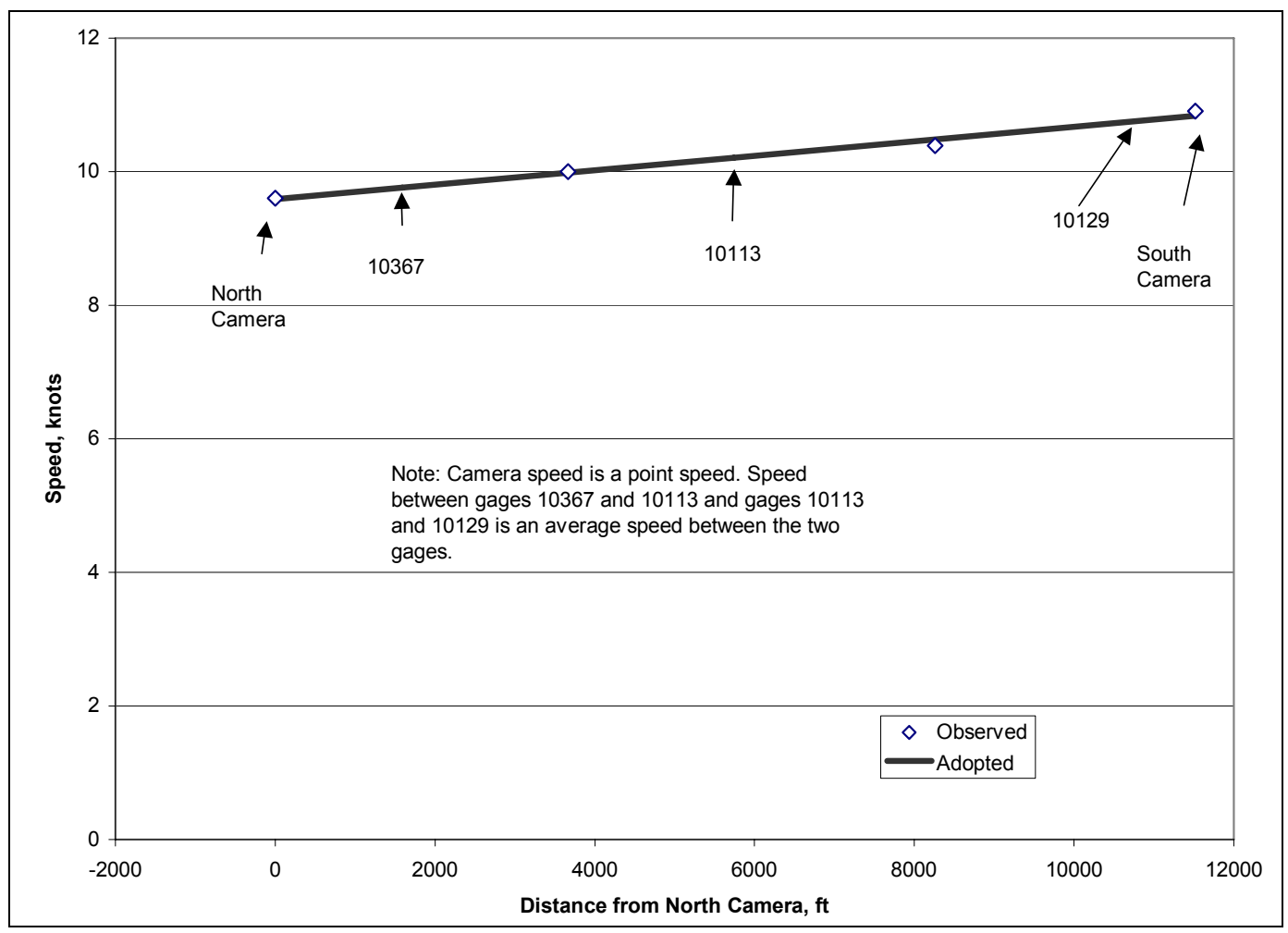

f. Eagle Subaru, outbound, 3 June 2003.

Figure 5. (Sheet 3 of 5). 


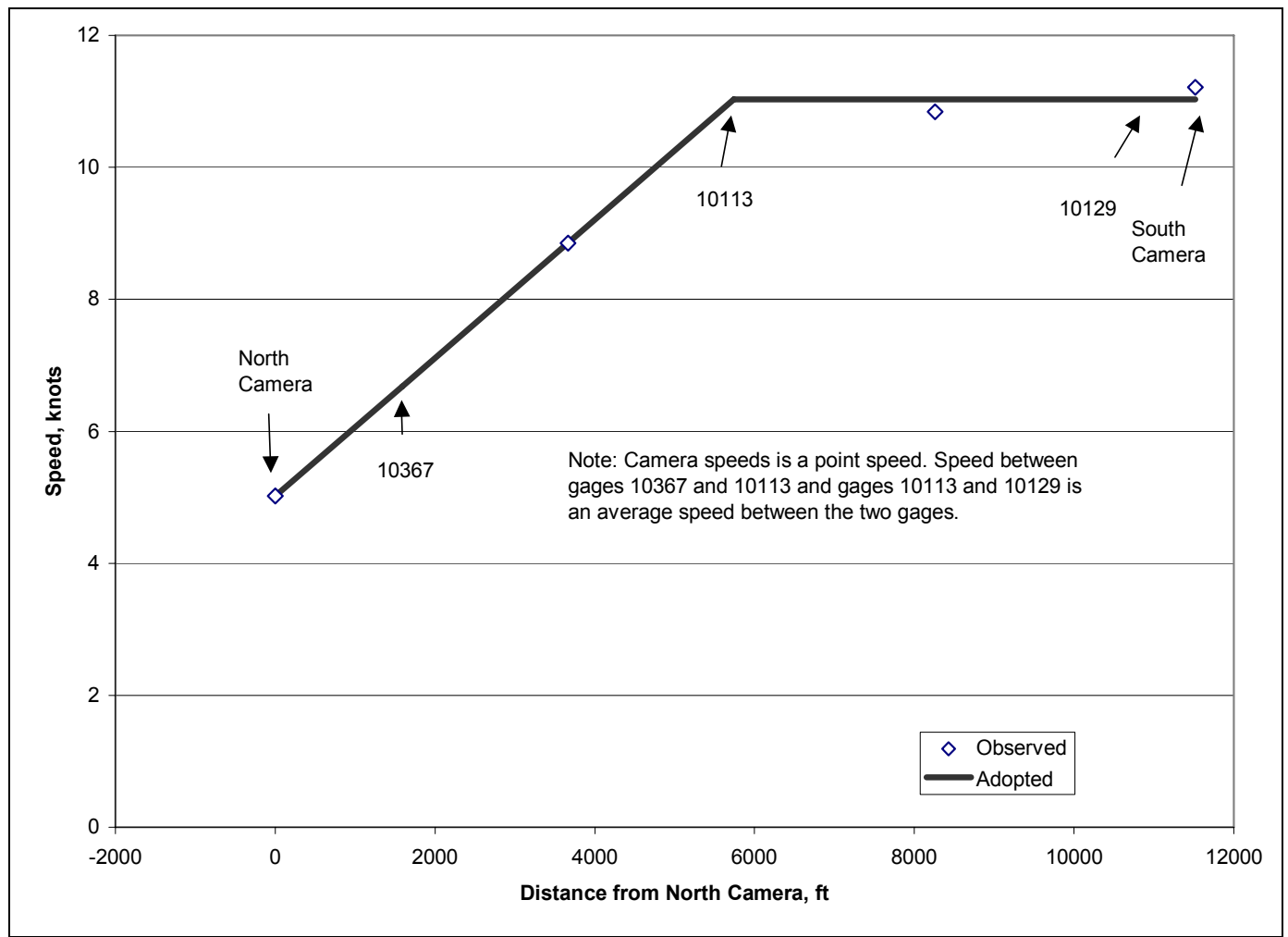

g. Lykes Ambassador, outbound, 4 June 2003.

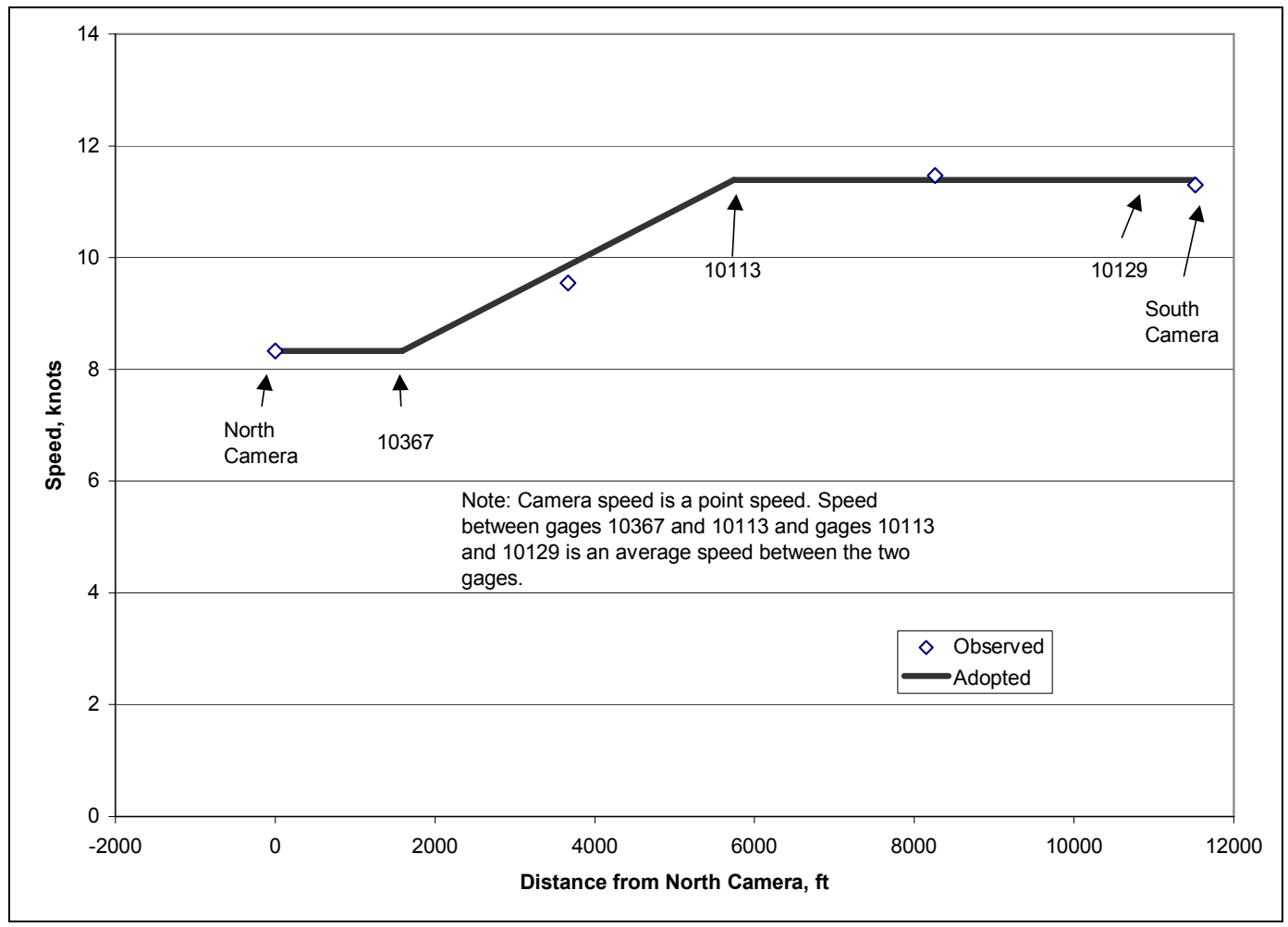

h. SKS Trent, outbound, 5 June 2003.

Figure 5. (Sheet 4 of 5). 


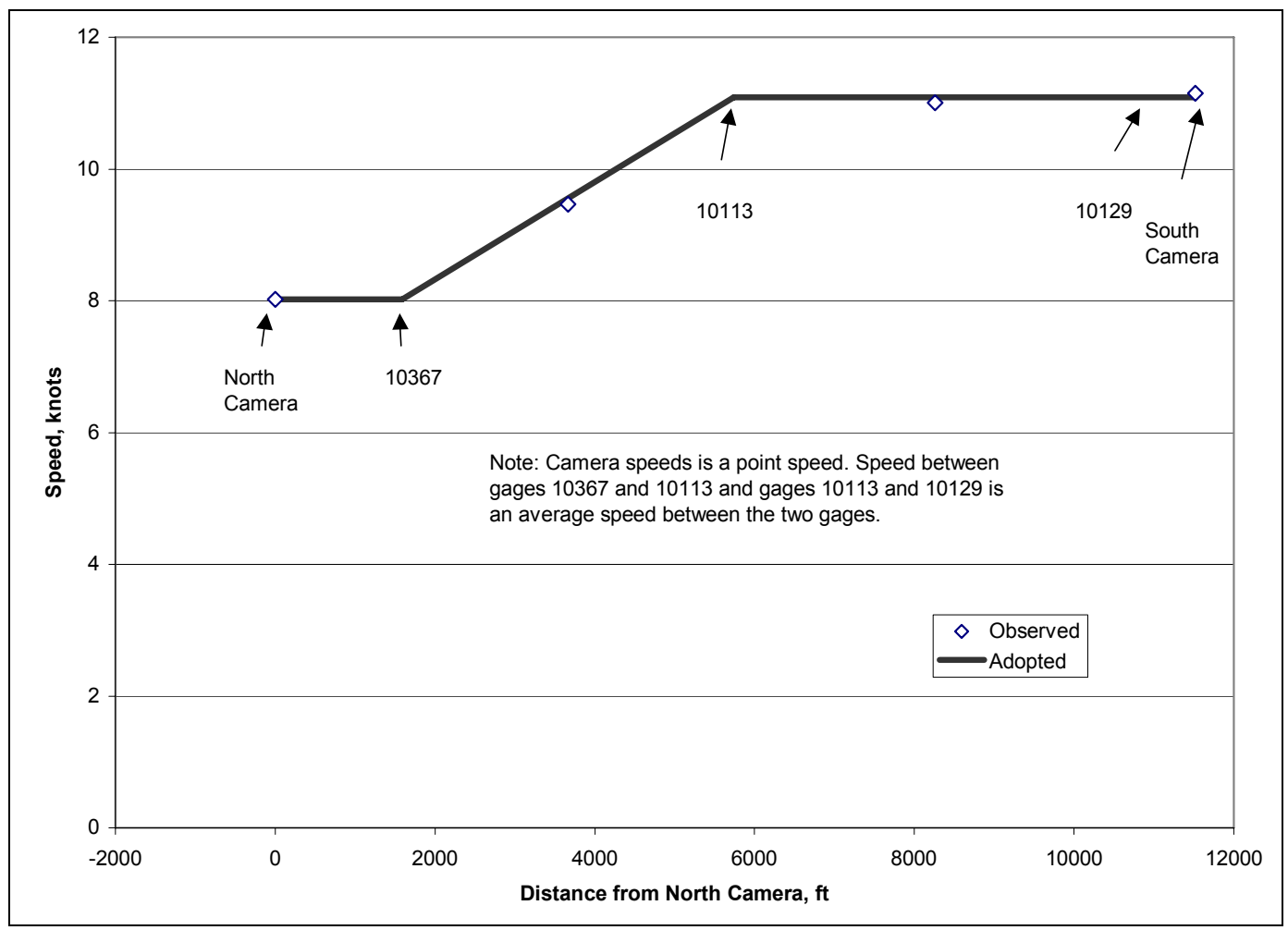

i. Gaz Progress, outbound, 5 June 2003.

Figure 5. (Sheet 5 of 5). 


\section{Numerical Model}

\section{HIVEL2D}

The numerical model HIVEL2D (Stockstill and Berger 19941) was modified and used to compute ship effects along the HSC and along the AIMB. HIVEL2D was developed to determine the currents and water surface in channels and near geometric features common in channels. The model solves the depth-averaged unsteady shallow-water equations implicitly, using finite elements. The modified version of the model includes the effect of a vessel in the channel. This version computes the long period drawdown wave generated from the vessel as well as the currents generated from the vessel passage.

The vessel is represented by imposing a pressure field similar to the vessel geometry (beam, length, and draft) and moving this pressure field each time step to correspond to the vessel's sailing speed. The computational mesh is constructed such that pressure gradients are applied across the bow, stern, and each side boundary in a manner to maintain the appropriate blockage area. The vessel is gradually accelerated to the desired sailing speed before it enters the area of concern.

The initial model represented the existing channel and mooring basin design and covered approximately 3 miles of the HSC, the AIMB, and a 2-mile width of the Galveston Bay west of the HSC. The gage locations used for comparison are shown in Figure 1 and additional information about gage location is provided in Table 1 . The ship effects determined at these locations provided the best information for future comparison of channel and mooring basin design changes.

\section{Grid Refinement}

The original grid shown in Figure 6 contained 41,507 elements and 34,858 nodes. A preliminary run with $S K S$ Trent outbound transit data was used to test the model. These results were then compared to the field

\footnotetext{
1 Stockstill, R. L., and R. C. Berger. 1994. HIVEL2D: A two-dimensional flow model for high-velocity channels. Technical Report REMR-HY-12. Vicksburg, MS: Waterways Experiment Station.
} 


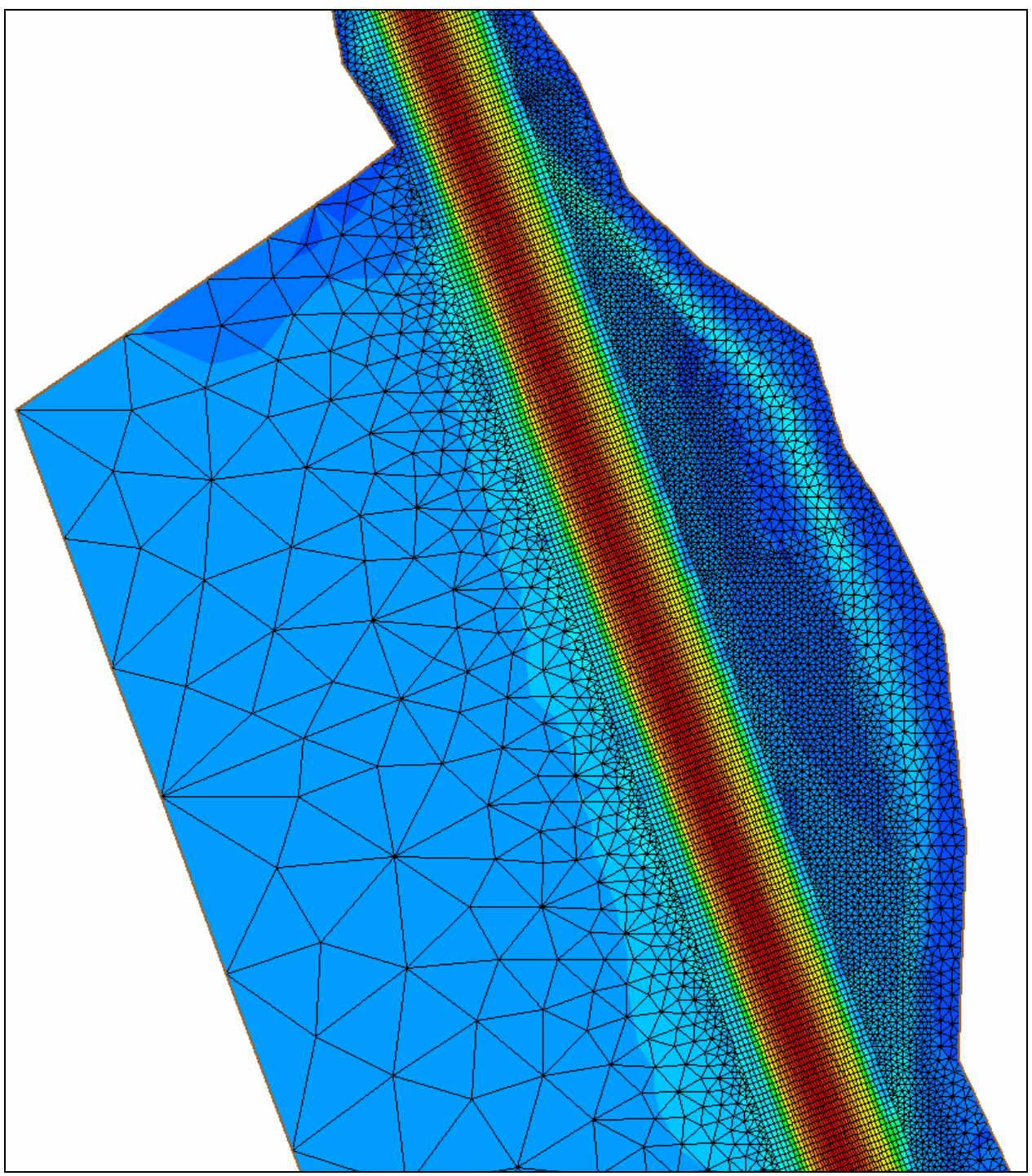

Figure 6. Detail of the original channel mesh.

study results. The time histories obtained from the field study for the $S K S$ Trent outbound are shown in Figure 3d. The time history for each gage is offset by $2 \mathrm{ft}$ on the vertical axis so each trace can be seen individually. The numerical model results for the SKS Trent outbound with the initial computational mesh are shown in Figure 7. The comparison shows good agreement between the field and numerical results. There were slight differences between the stern wave at gage 10347 and the front wave at gage 10349. Comparison of the magnitude and timing of the drawdown was in very good agreement at the gages. 


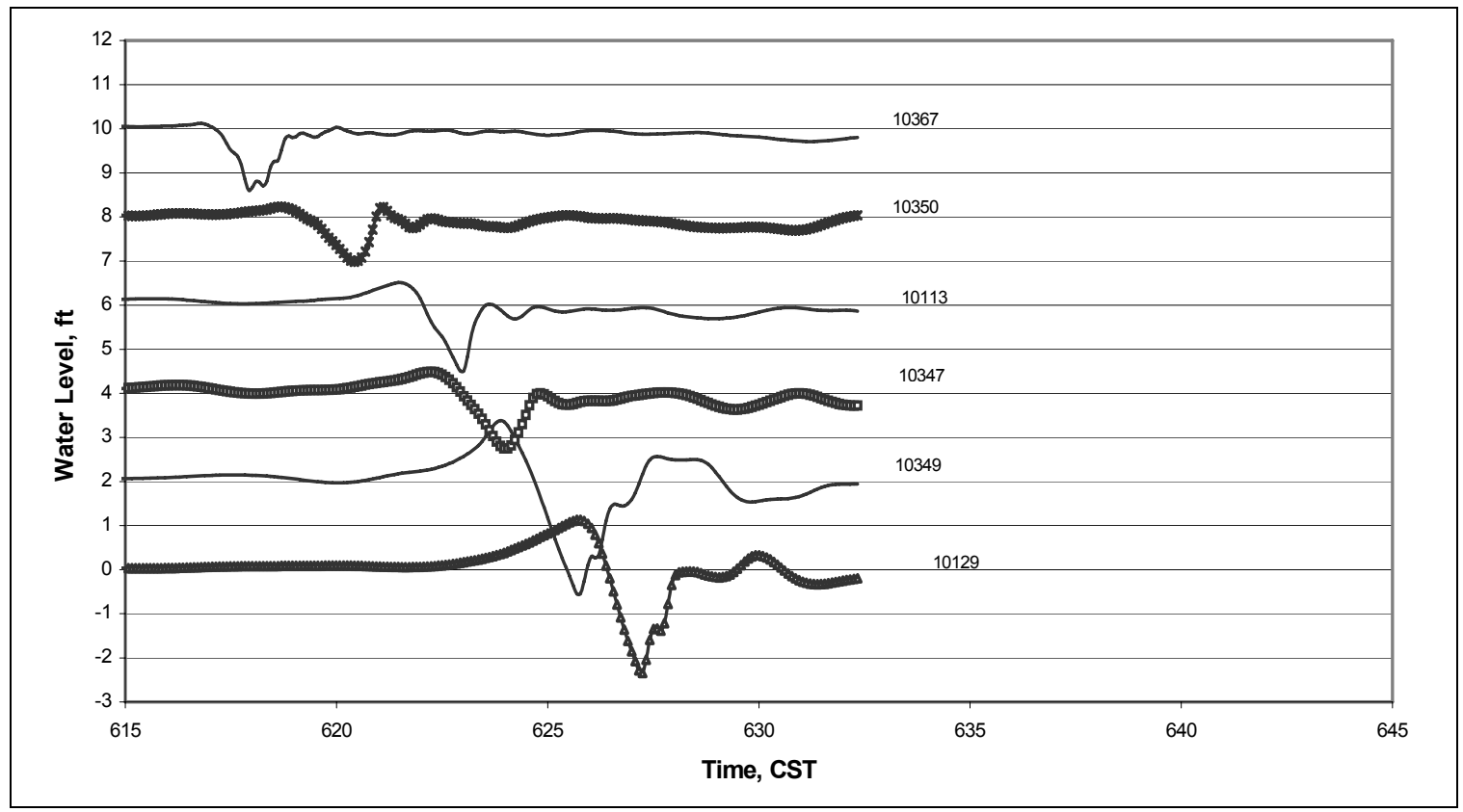

Figure 7. HIVEL2D computed water-level, SKS Trent, outbound, run 79565.

Mesh convergence was then performed. Mesh convergence is a process of refining the mesh until the model results do not change significantly with additional refinement. The refined model shown in Figure 8 contained 81,462 elements and 53,102 nodes. Results for the SKS Trent outbound with the refined model are shown in Figure 9. The refined grid was then modified to include more of the bay west of the HSC, as shown in Figure 10. The final mesh contained 88,906 elements and 56,452 nodes.

\section{Validation}

The final model was validated by comparing time histories of the water surfaces measured during the field study to the computed water surfaces at similar locations in the numerical model. These comparisons were made at the six gage locations for four ship transits, inbound and outbound transits with SKS Trent, outbound with the Eagle Subaru, and inbound with the Berge Kobe. The length, beam and draft of each of these ships are provided in Table 4. The time histories obtained from the field study are shown in Figures 2a, 2d, 3b, and 3d. The computed results for the SKS Tent outbound are shown in Figure 11 and Figure 12 shows the time histories for the SKS Trent inbound. The time histories of the water level for the Eagle Subaru outbound are shown in Figure 13 and the Berge Kobe inbound in Figure 14. 


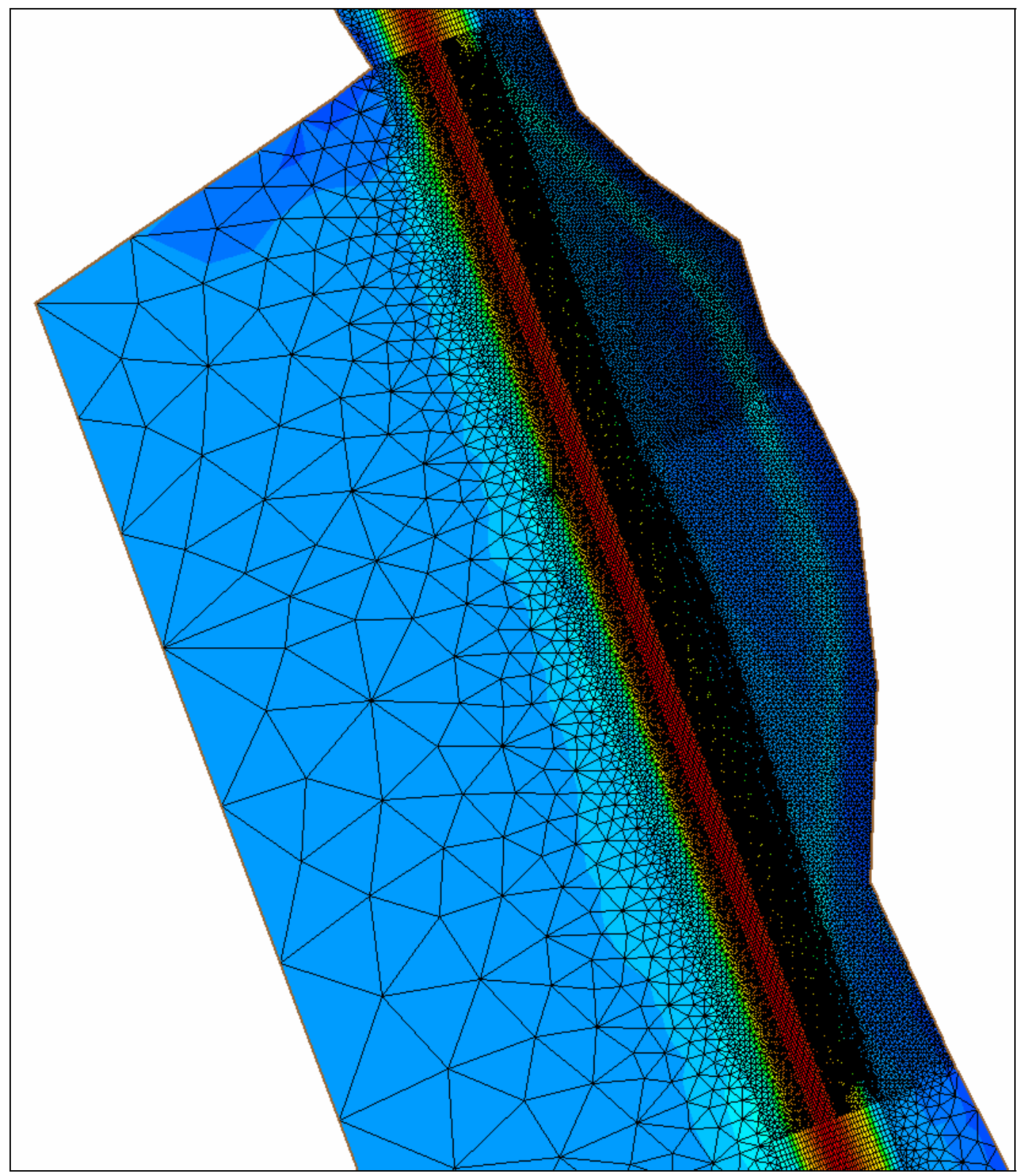

Figure 8. Detail of the refined channel mesh. 


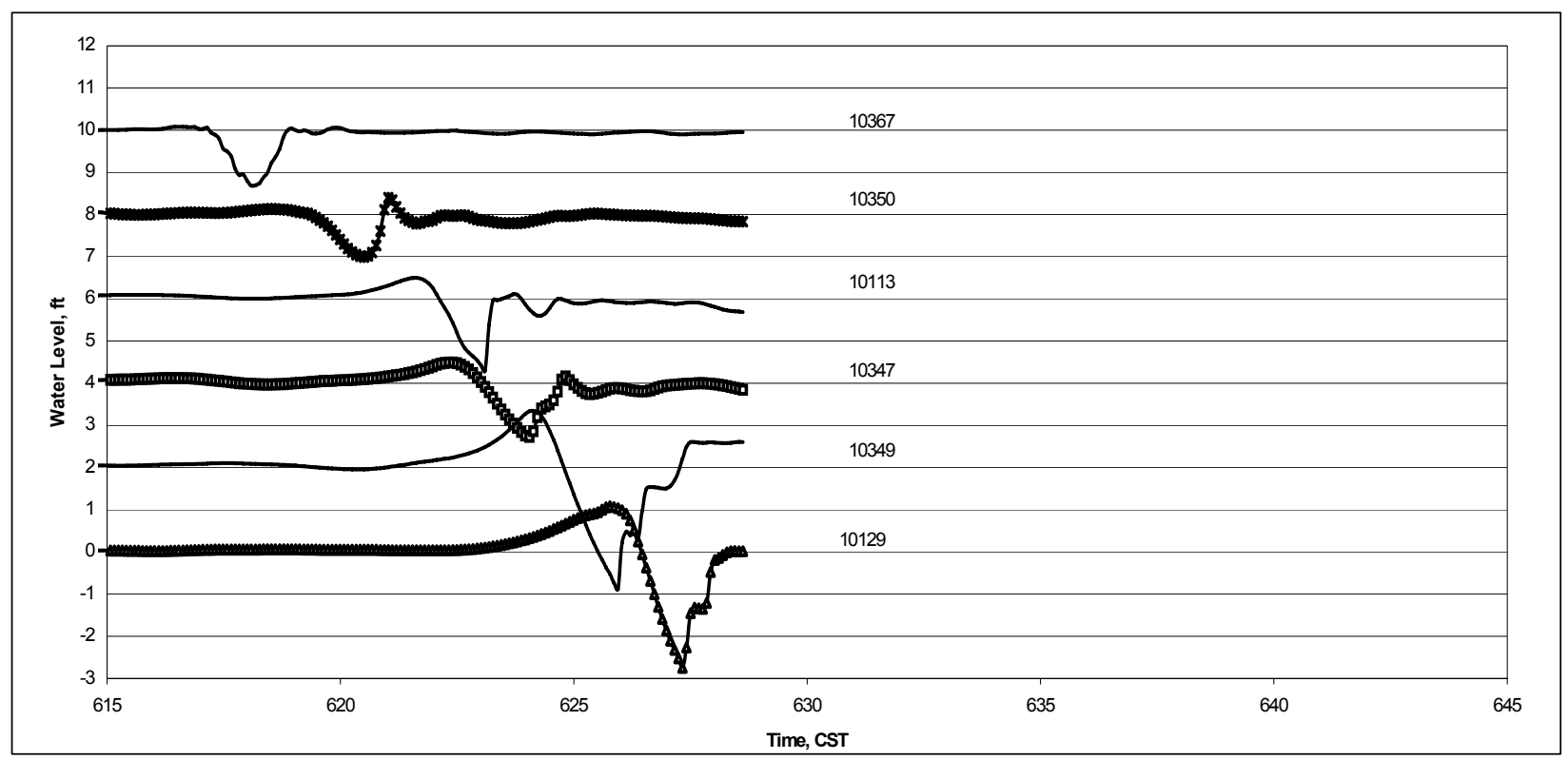

Figure 9. HIVEL2D computed water-level, SKS Trent, outbound, run 80561.

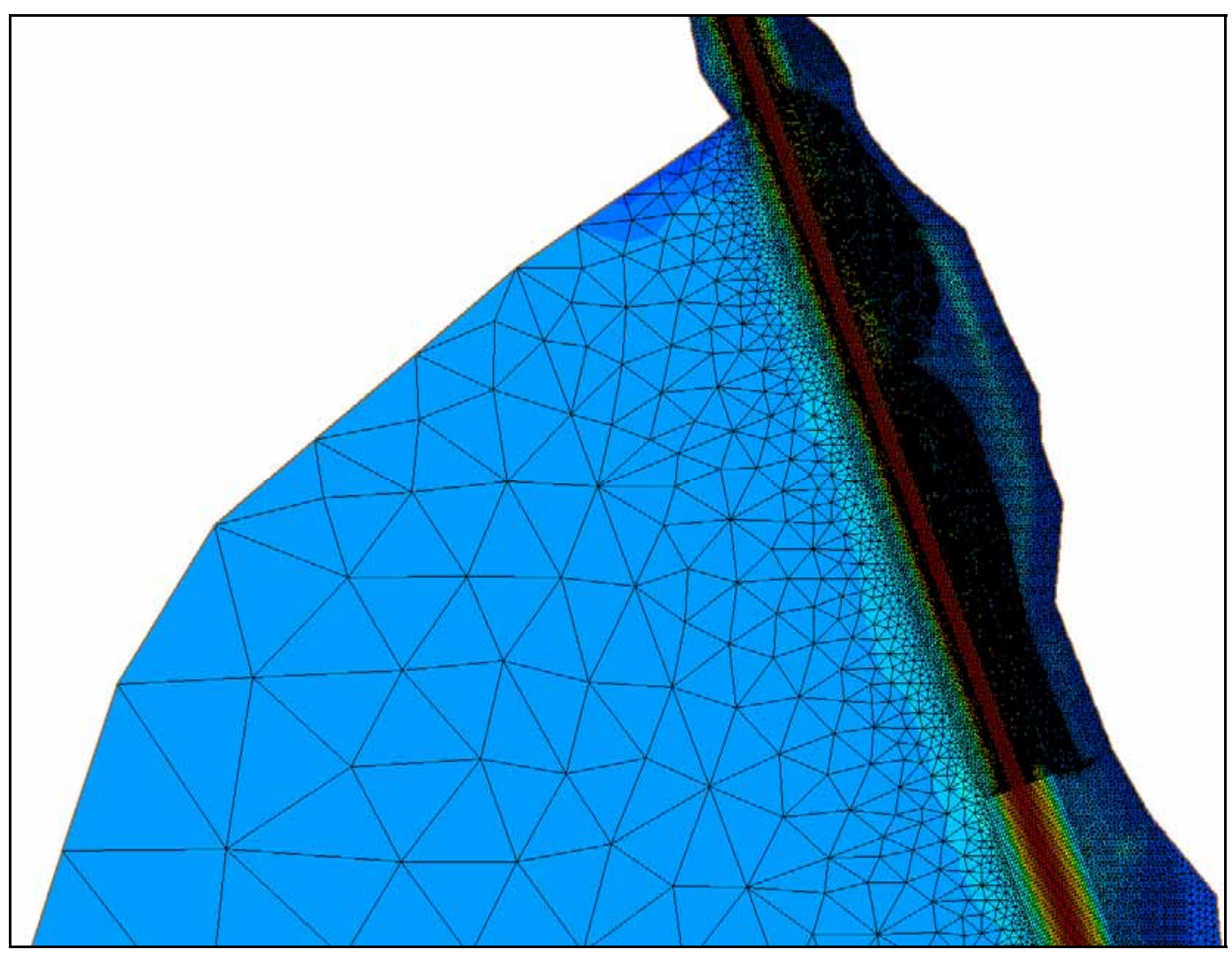

Figure 10. Detail of the refined mesh including the bay. 
Table 4. General ship info.

\begin{tabular}{|l|l|l|l|l|}
\hline Ships to be modeled & $\begin{array}{l}\text { Length } \\
\mathrm{ft}\end{array}$ & $\begin{array}{l}\text { Beam } \\
\mathrm{ft}\end{array}$ & $\begin{array}{l}\text { Draft } \\
\mathrm{ft}\end{array}$ & $\begin{array}{l}\text { WSE } \\
\mathrm{ft}, \mathrm{MLT}\end{array}$ \\
\hline SKS Trent - Outbound & 800 & 138 & 30 & 1.46 \\
\hline Eagle Subaru - Outbound & 810 & 138 & 28 & 2.46 \\
\hline Berge Kobe - Inbound & 721 & 126 & 35 & 2.61 \\
\hline SKS Trent - Inbound & 800 & 138 & 32 & 2.46 \\
\hline \multicolumn{7}{|l|l|}{ Adopted Measurements For Simulation Purposes } \\
\hline SKS Trent - Outbound & 800 & 140 & 30 & 1.46 \\
\hline Eagle Subaru - Outbound & 810 & 140 & 28.1 & 2.46 \\
\hline Berge Kobe - Inbound & 721 & 140 & 31.5 & 2.61 \\
\hline SKS Trent - Inbound & 800 & 140 & 31.5 & 2.46 \\
\hline
\end{tabular}

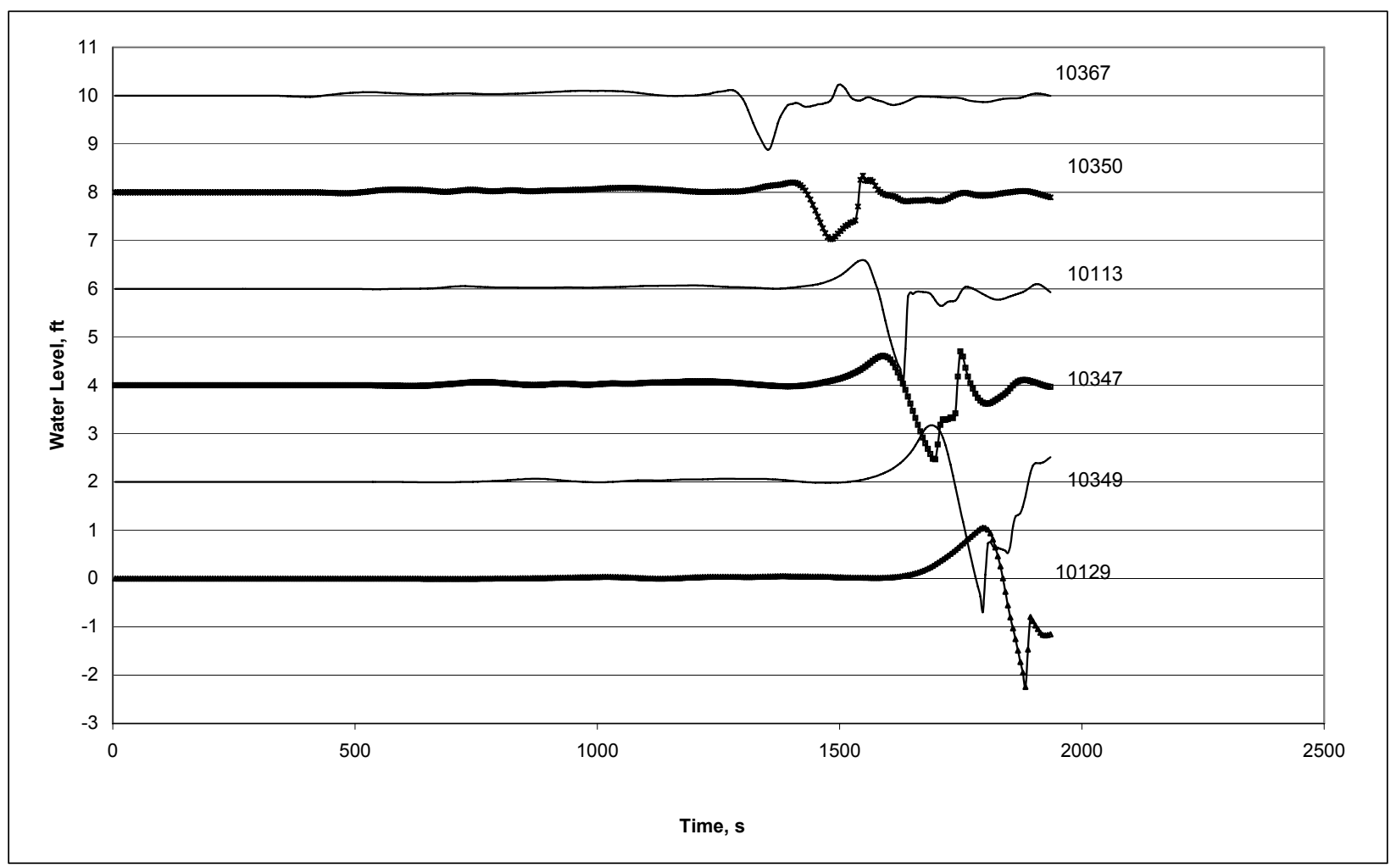

Figure 11. SKS Trent, outbound, all gages (computed - final model). 


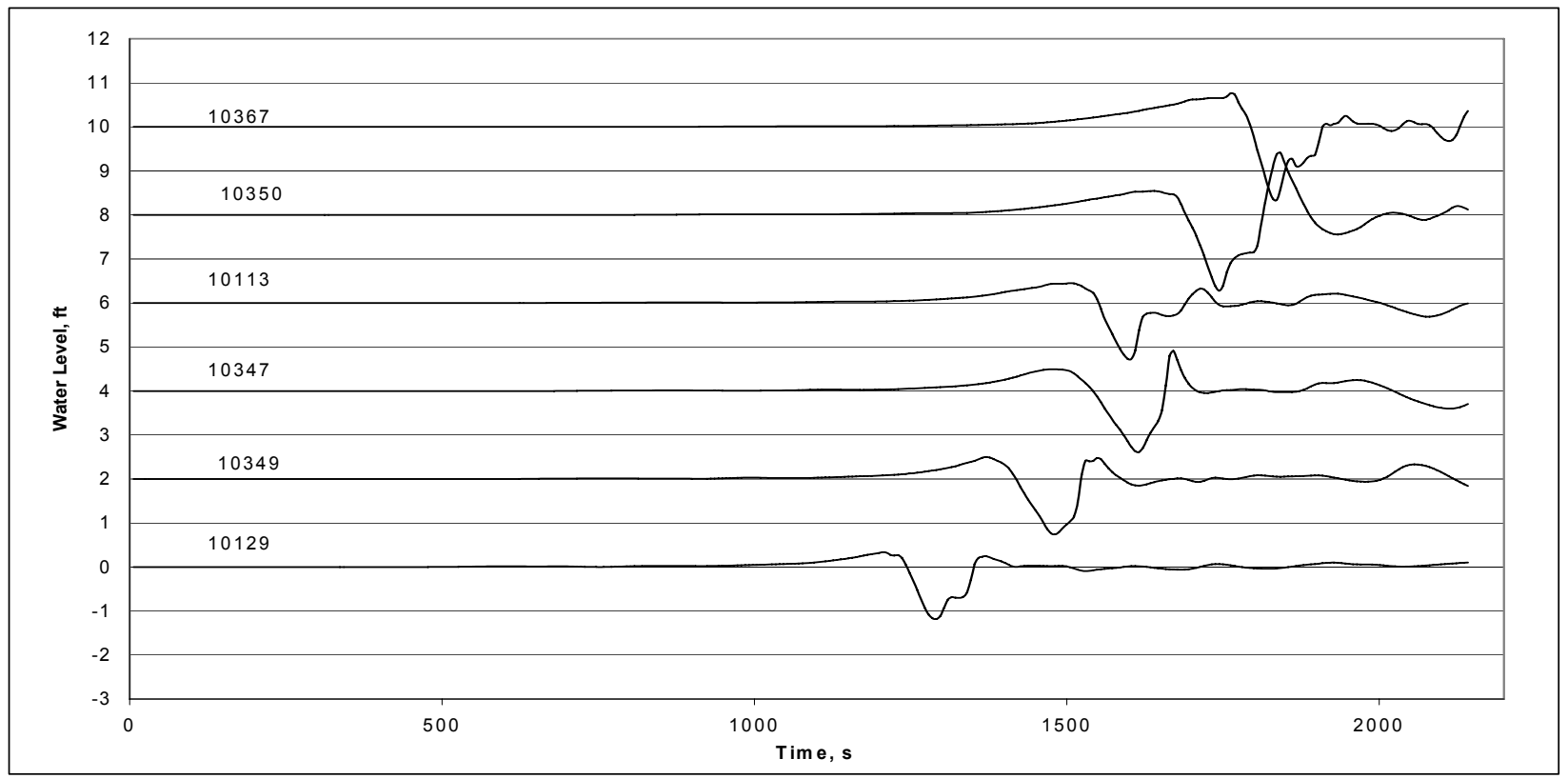

Figure 12. SKS Trent, inbound, all gages (computed).

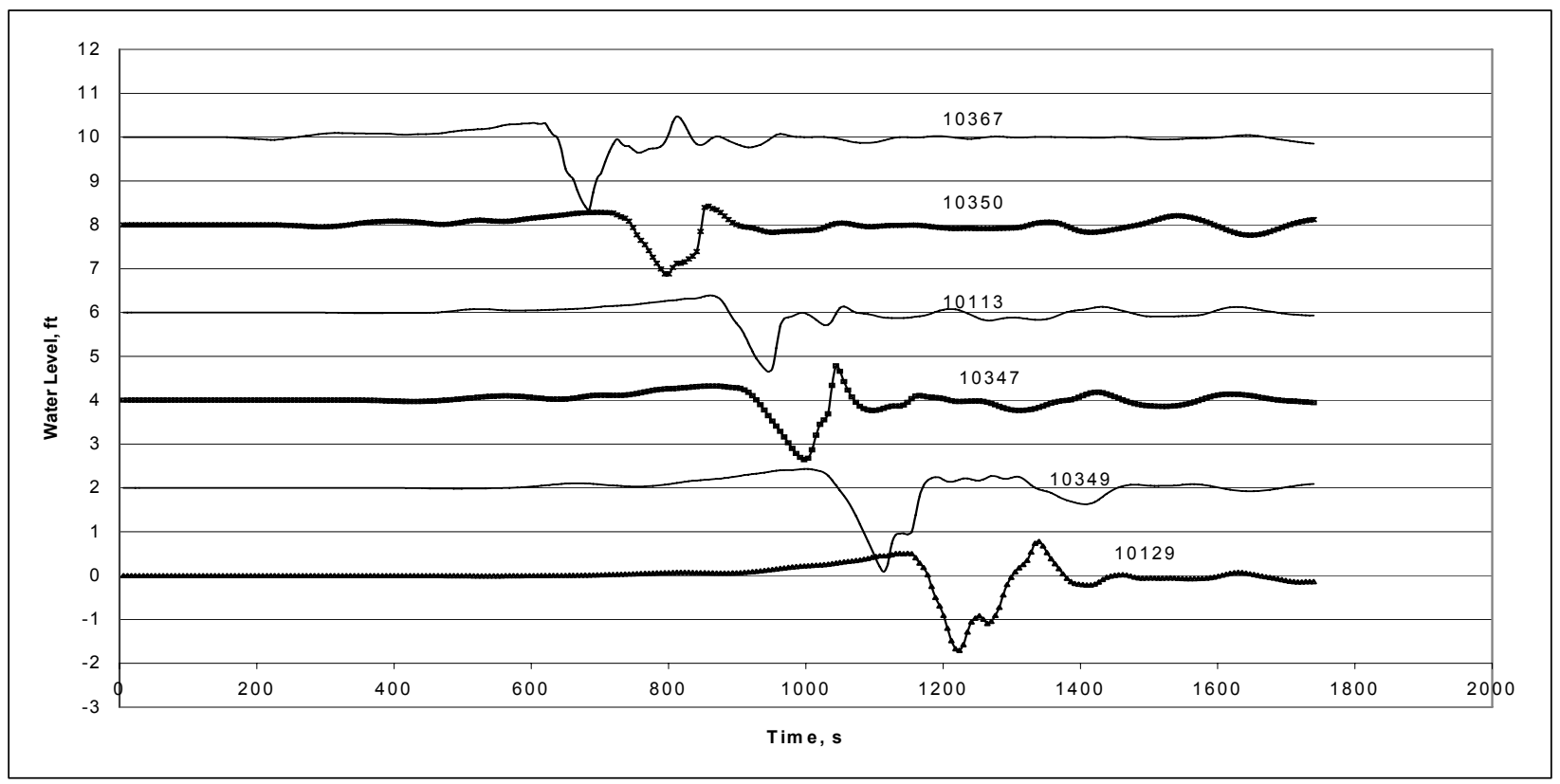

Figure 13. Eagle Subaru, outbound, all gages, (computed). 


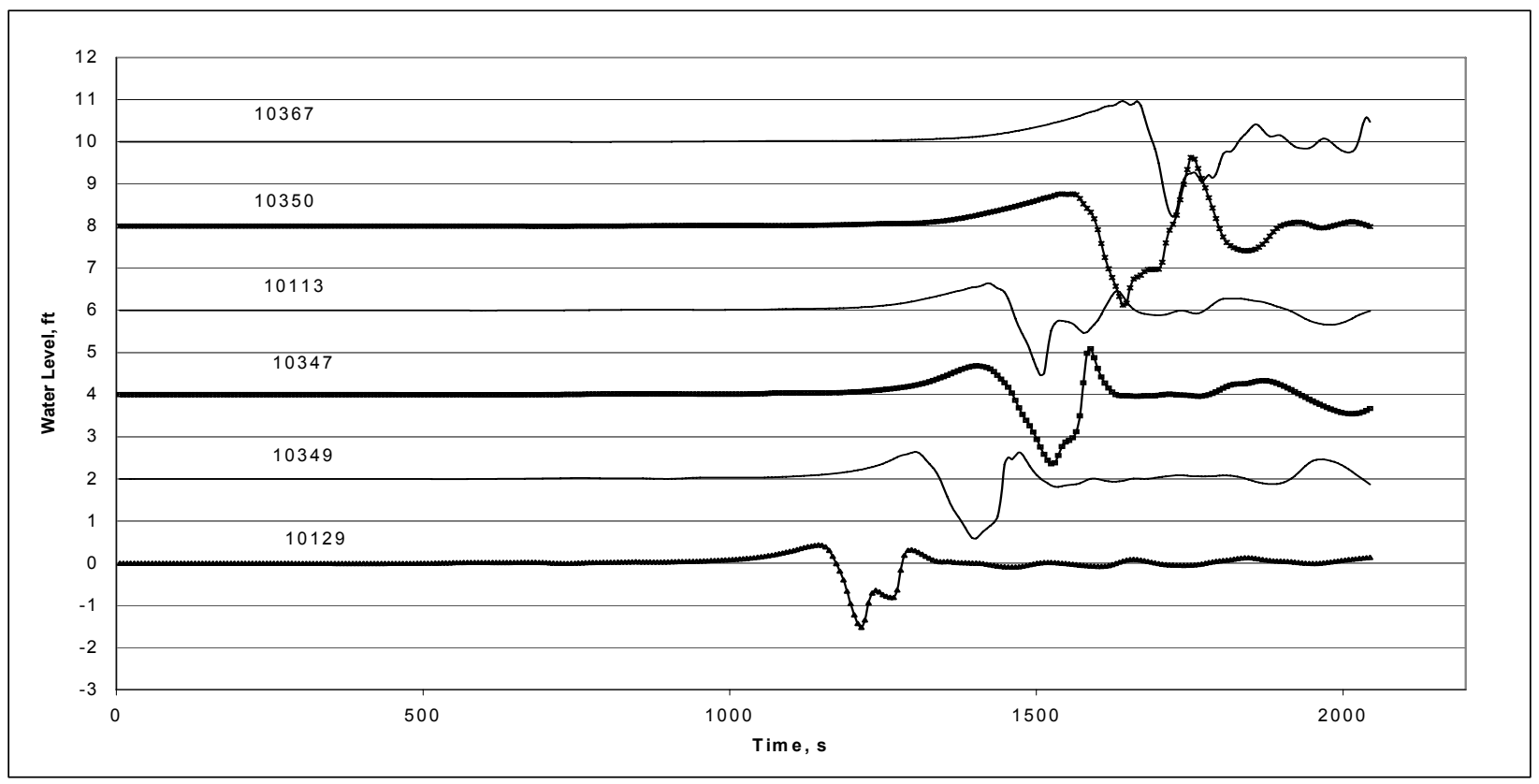

Figure 14. Berge Kobe, inbound, all gages (computed).

Comparison of the water-surface time histories between the field experiments and the numerical model showed very good agreement. The magnitude and timing of the computed drawdown was close enough to feel confident that existing conditions were reproduced and that changes to the geometry could be modeled accurately using HIVEL2D. The numerical model was therefore validated. 


\section{Numerical Model Alternative Designs Results}

\section{New Channel}

The new channel for the mooring basin was added to the computational mesh. The channel invert along the mooring area was $150-\mathrm{ft}$ wide with a bottom elevation of $-12 \mathrm{ft}$. The coordinates close to where the north centerline of the mooring basin channel intersected the north centerline of the HSC were $3244725.6,13816189.0$. The coordinates close to where the south centerline of the mooring basin channel intersected the south centerline of the HSC were $3248214.4,13806732.0$. A channel invert layout and mooring locations for the new channel are shown in Figure 15. The mooring positions were located on both sides of the channel. Each of the six locations represented a group of moored barges $700 \mathrm{ft}$ long by $52 \mathrm{ft}$ wide.

\section{Dike Designs}

Numerical simulations to determine water levels in the basin during ship transits were performed for several dike configurations. The length, location, and layout were varied to determine the design that was the most successful in reducing ship effects in the AIMB during ship transits in the HSC. Dike lengths of 2300 and $3300 \mathrm{ft}$, with the centerline of the dike located at distances of 250 and $500 \mathrm{ft}$ from the centerline of the mooring basin channel, were evaluated. The initial evaluations were based on water levels in the mooring basin during an outbound transit of a ship that represented the dimensions of the SKS Trent. The length was $800 \mathrm{ft}$, the width was $140 \mathrm{ft}$, and the draft was $30 \mathrm{ft}$. The $S K S$ Trent outbound reached a speed of 11.4 knots when adjacent to the mooring basin (Figure $5 \mathrm{~h}$ ).

\section{Without Dike}

A baseline condition was simulated without a dike to determine the water levels with no protective structure to minimize ship effects. The locations where the water levels were determined are shown in Figure 15. The locations were designated E1, E2, E3, and E4 for those located on the east side of the AIMB channel and $\mathrm{W}_{1}, \mathrm{~W}_{2}, \mathrm{~W}_{3}$, and $\mathrm{W}_{4}$ for those located on the 


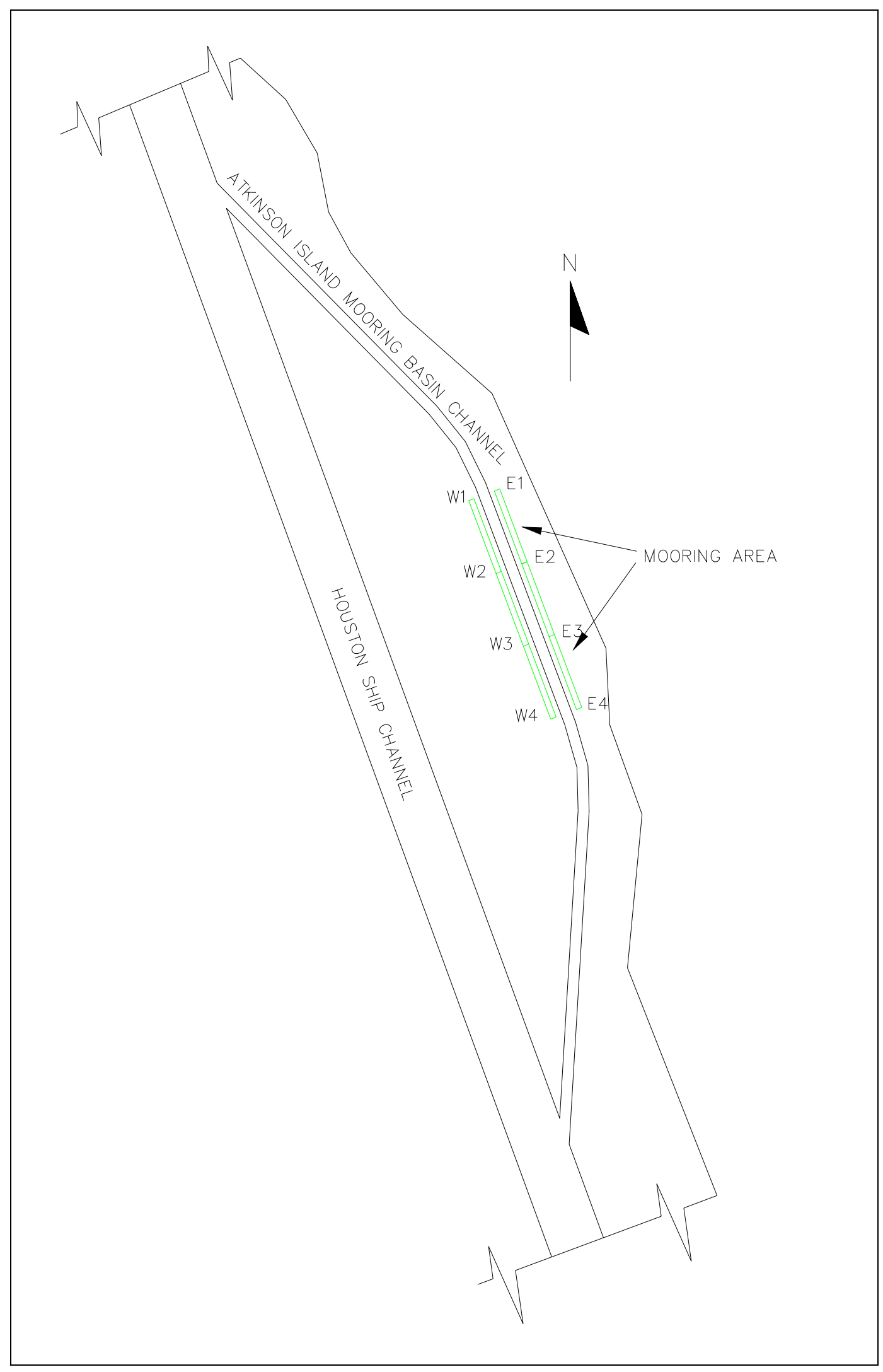

Figure 15. Channel invert layout and mooring locations. 
west side. Time histories of the water level were plotted for these locations from the time the ship was approximately $1800 \mathrm{ft}$ above the north entrance of the basin (time 1161 seconds) until the time the ship was well below the south end of the basin (time 1935 seconds).

A moored tow was simulated at location E3-E4 by imposing a pressure field that represented a vessel geometry of 700 by $52 \mathrm{ft}$ with a $9-\mathrm{ft}$ draft. The time histories of the water levels with the no dike condition are shown in Figure 16 between the times of 1161 and1935 seconds. The initial watersurface elevation was set at $1.46 \mathrm{ft}$ for all simulations. The maximum water level computed in the basin for the eight locations was elevation $2.3 \mathrm{ft}$ and occurred at location $\mathrm{E} 4$ at time 1599.6 seconds. The minimum water level was at elevation $-0.29 \mathrm{ft}$ and also occurred at location $\mathrm{E} 4$ at time 1689.9 seconds.

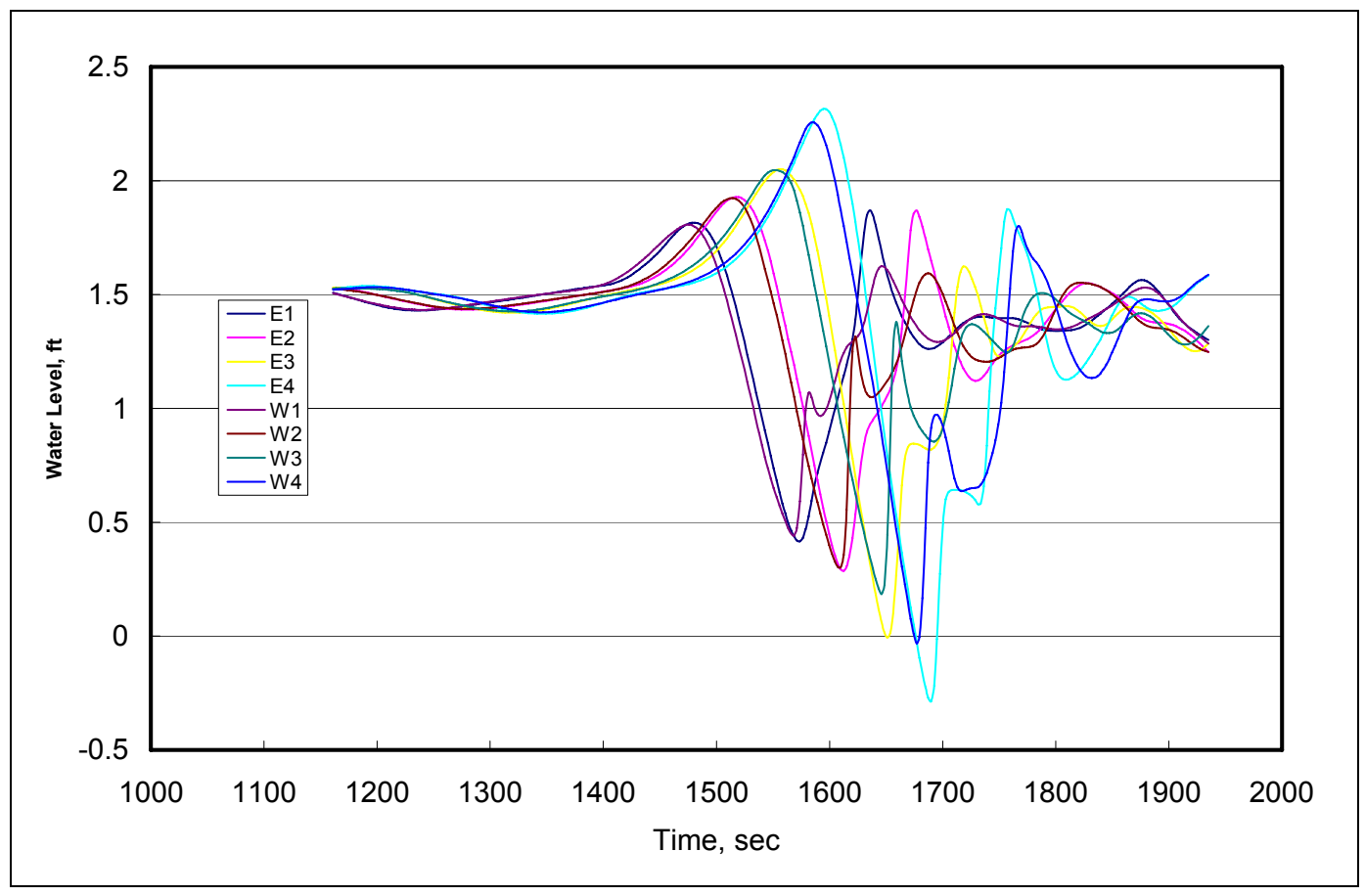

Figure 16. Water levels ( $\mathrm{ft}$ ) in basin without dike with a tow moored between E3 and E4 with SKS Trent outbound.

The maximum water-level variations for the eight selected locations occurred in the south end of the basin at locations E4 and W4. The water-level difference from one end of the moored barges to the other end, as well as the water-level difference across the moored barges, is important to know because these differences will generate forces in the mooring lines. The end-to-end difference in the north-south direction was designated the 
longitudinal water-level difference and the east-west water-level difference across the moored barges was designated the lateral difference. The maximum longitudinal water-level difference for the without dike condition was $1.1 \mathrm{ft}$ at time 1689.9 seconds and occurred between locations E3 and E4. The negative value indicates the water level was higher on the south measurement location than the north measurement location. For example, if for a given time, the water level is higher at location E4 than E3, the difference is reported as a negative water-level difference. The maximum lateral water-level difference was $1.2 \mathrm{ft}$ at time 1689.9 seconds and occurred between locations E4 and W4. Figure 17a shows the longitudinal water-level differences at the eight mooring locations and Figure $17 \mathrm{~b}$ shows lateral water-level differences. The negative value indicates that the water level was higher on the west side of the AIMB channel (W4) than the east side (E4). Table 5 provides the water-level difference information for the without dike condition, newgrid_wodike_wtow, along with the results from the other simulations. Because the maximum longitudinal waterlevel differences occurred at locations $\mathrm{E}_{3}$ and $\mathrm{E}_{4}$ and the maximum lateral water-level differences occurred at locations $\mathrm{E}_{4}$ and $\mathrm{W}_{4}$, these were selected for comparison with subsequent simulations with the SKS Trent outbound.

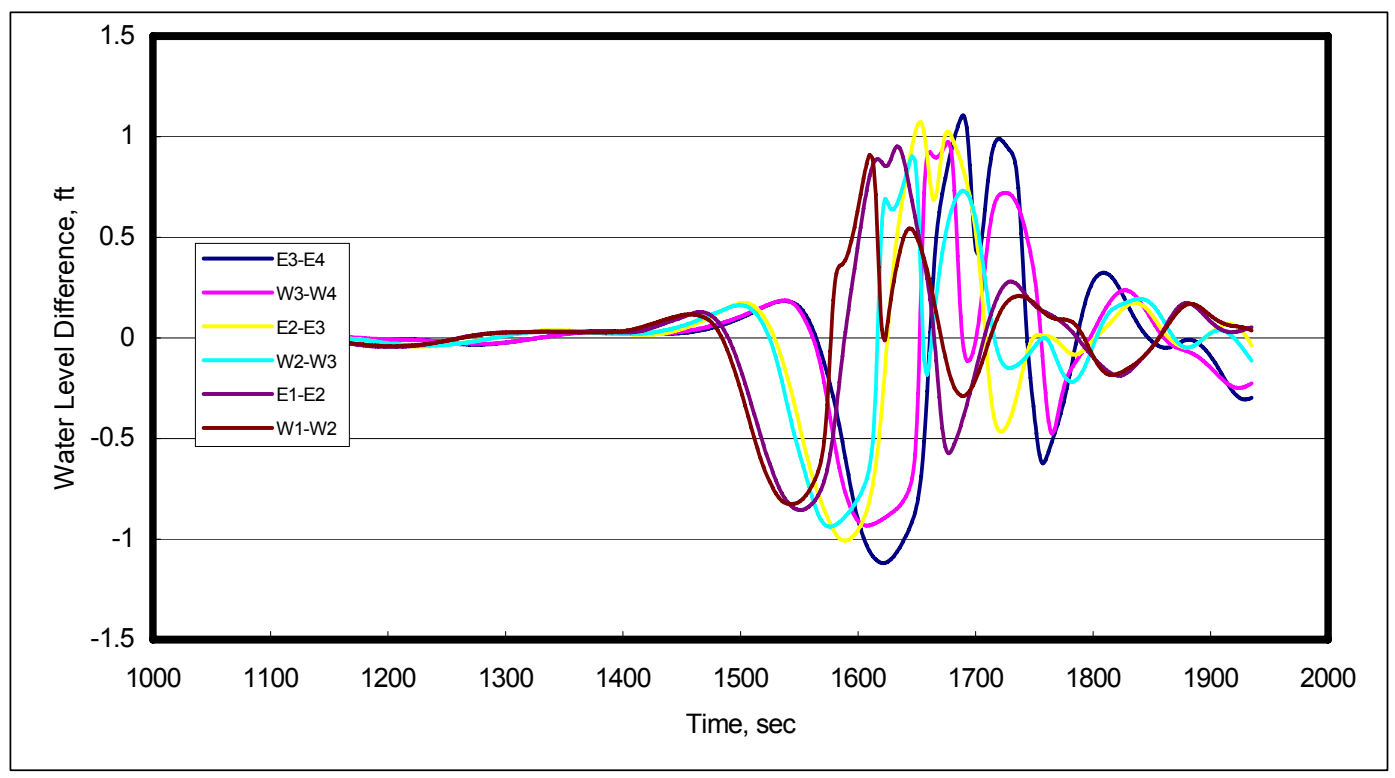

a. Longitudinal differences.

Figure 17. Water-level differences in basin without dike with tow moored at E3-E4, with SKS Trent outbound (Continued). 


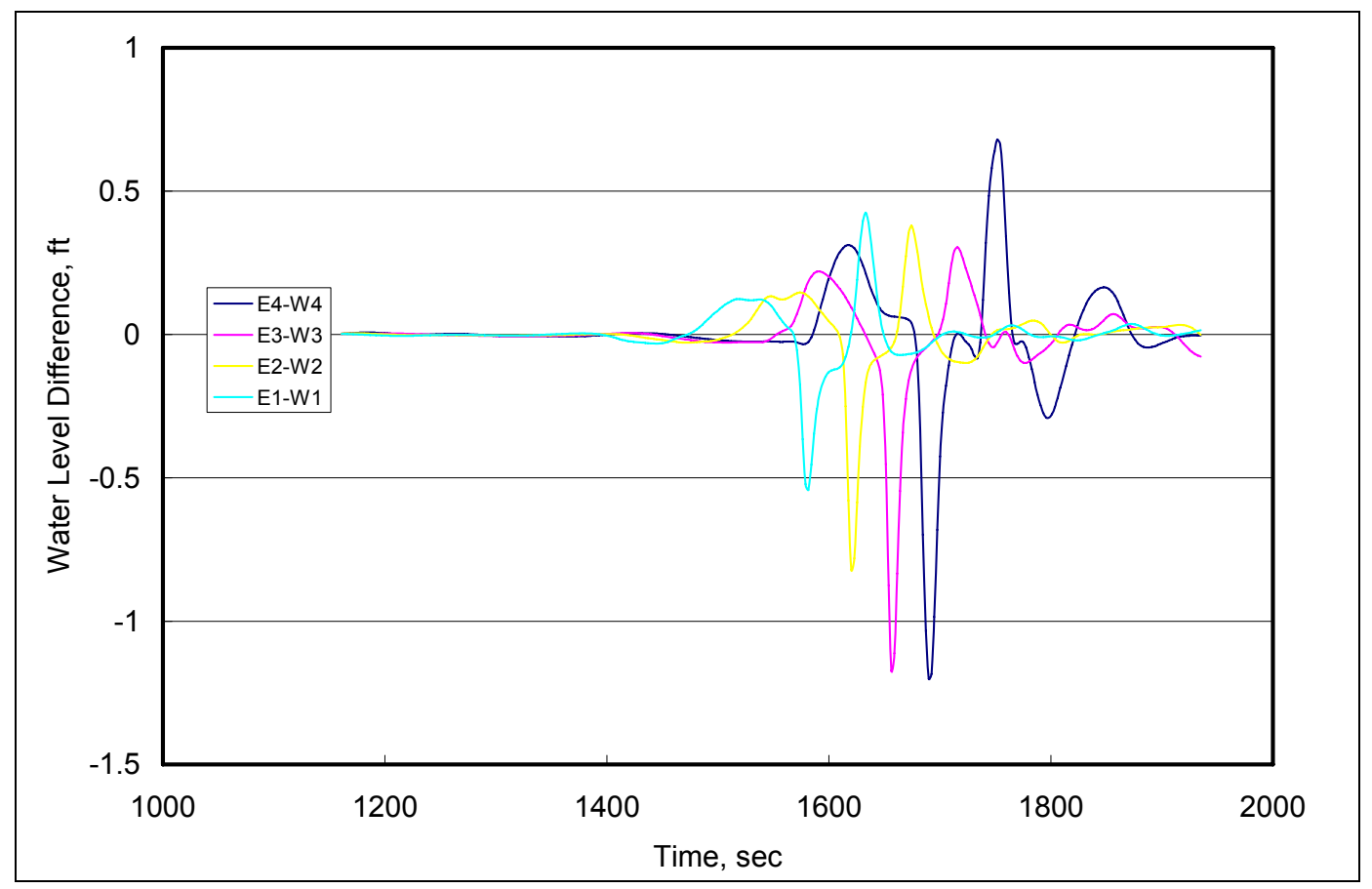

b. Lateral differences.

Figure 17. (Concluded).

Table 5. Maximum water-level differences in mooring basin in feet, SKS Trent outbound.

\begin{tabular}{|l|l|l|l|l|}
\hline Grid_Design & $\begin{array}{l}\text { Longitudinal } \\
\text { (North to South) }\end{array}$ & Location:time & $\begin{array}{l}\text { Lateral (East to } \\
\text { West) }\end{array}$ & Location:time \\
\hline Newgrid_wodike_wtow & 1.10 & E3-E4:1689.9 & $-1.20 *$ & E4-W4:1689.9 \\
\hline Newchan_bw1 & 1.10 & E3-E4:1720.9 & 0.44 & E4-W4:1777.6 \\
\hline Newgrid_bw2 & 1.08 & E3-E4:1723.44 & -0.18 & E4-W4:1800.84 \\
\hline Newchan_bw2 & 1.05 & E3-E4:1731.18 & -0.14 & E4-W4:1811.16 \\
\hline Newchan_bw3 & 0.99 & E3-E4:1718.3 & 0.48 & E4-W4:1777.6 \\
\hline Newchan_bw4 & 0.99 & E3-E4:1733.76 & -0.21 & E4-W4:1790.52 \\
\hline Newchan_bw5 & 1.01 & E3-E4:1228.6 & 0.22 & E4-W4:1790.5 \\
\hline Newchan_bw6 & 0.78 & E3-E4:1754.4 & 0.08 & E4-W4:1922.1 \\
\hline Newgrid_bw6 & 0.80 & E3-E4:1751.8 & -0.11 & E4-W4:1922.1 \\
\hline Newchan_bw7 & 0.82 & E3-E4:1736.3 & -0.19 & E4-W4:1811.2 \\
\hline Newchan_bw8 & 0.89 & E3-E4:1754.4 & -0.06 & E4-W4:1834.4 \\
\hline Newgrid_bw8 & 0.93 & E3-E4:1746.7 & -0.06 & E4-W4:1829.2 \\
\hline Newgrid_bw9 & 0.41 & E3-E4:1764.7 & -0.15 & E4-W4:1916.9 \\
\hline Newgrid_bw10 & 0.55 & E3-E4:1824.1 & -0.25 & E4-W4:1924.7 \\
\hline Newgrid_bw11 & 0.48 & E3-E4:1749.2 & -0.13 & E4-W4:1834.4 \\
\hline Newgrid_bw12 & 0.99 & E3-E4:1741.5 & 0.11 & E4-W4:1798.26 \\
\hline Newgrid_bw13 & 0.59 & E3-E4:1749.2 & 0.16 & E4-W4:1790.52 \\
\hline Newgrid_bw14 & 0.54 & E3-E4:1749.2 & -0.14 & E4-W4:1834.4 \\
\hline * Negative indicates water level on west side is higher than east side. & \\
\hline
\end{tabular}




\section{Dikes bw1 - bw4}

The first dike design evaluated was a 2300-ft long dike adjacent to the AIMB with its centerline of the dike located $250 \mathrm{ft}$ west of the centerline of the mooring basin channel as shown in Figure 18. The dike width was $20 \mathrm{ft}$ and was modeled with the crown elevation above the water surface. The longitudinal water-level differences inside the mooring basin, at E3 and $\mathrm{E} 4$, and the lateral water-level differences, at $\mathrm{E}_{4}$ and $\mathrm{W}_{4}$, are listed in Table 5. The dike reduced the lateral water-level difference from -1.2 to $0.44 \mathrm{ft}$. The longitudinal water-level difference from the without dike condition did not change.

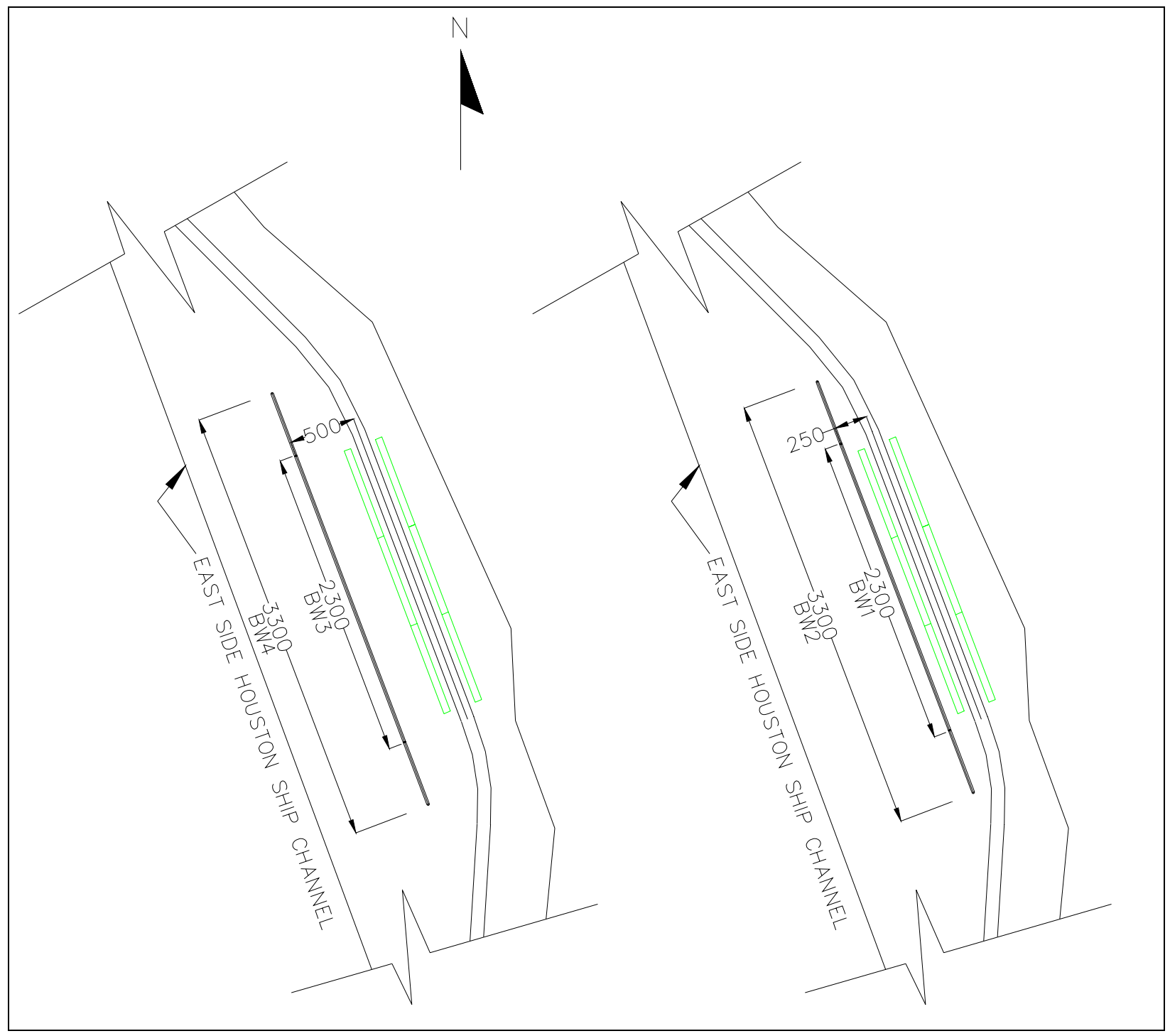

Figure 18. Layout for dikes bw1-bw4. 
The dike length was increased from 2300 to $3300 \mathrm{ft}$ to form dike bw2. The centerline distance from dike to the AIMB channel remained at $250 \mathrm{ft}$. Simulations were performed with the new channel grid (new_chan) and with the channel refined (new_grid). The water-level differences were similar for each grid, as shown in Table 5. The longer dike helped to further reduce the lateral water-level difference and there was still no change in the longitudinal water-level difference.

A simulation was performed with a $2300-\mathrm{ft}-$ long dike with its centerline located $500 \mathrm{ft}$ from the centerline of the AIMB channel. This dike was designated bw3 and there was a slight decrease in the longitudinal waterlevel difference (1.05 to $0.99 \mathrm{ft}$ ). The lateral water-level difference was similar to that with dike bw1as shown in Table 5. A 3330-ft long dike was evaluated with its centerline located $500 \mathrm{ft}$ from the centerline of the AIMB channel, dike bw4. The lateral water-level difference was reduced and there was still no change in the longitudinal water-level difference. The longer dike with a centerline located $500 \mathrm{ft}$ from the centerline of the AIMB channel was more effective in reducing the lateral ship effects, although additional changes were needed to help reduce the longitudinal ship effects.

\section{Dikes bw5 and bw6}

End sections were placed on the 2300 - $\mathrm{ft}$ dike located $250 \mathrm{ft}$ from the AIMB channel centerline to form dike bw5 shown in Figure 19. The maximum lateral water-level difference was reduced from dike bw1 without end sections, although, as seen in Table 5 , no reduction in the maximum longitudinal water-level difference occurred. End sections were then added to the 3300-ft dike to form dike bw6 shown in Figure 19. Some reduction in the maximum longitudinal water-level difference was achieved from dike bw5 as shown in Table 5 (1.01 to $0.78 \mathrm{ft}$ ) and the lateral water-level difference was also reduced ( 0.22 to $0.08 \mathrm{ft}$ ). The simulation with the new grid showed similar results. The water levels at locations E1, E2, E3, E4, W1, $\mathrm{W}_{2}, \mathrm{~W}_{3}$, and $\mathrm{W}_{4}$ inside the basin with the refined grid are shown in Figure 20. 


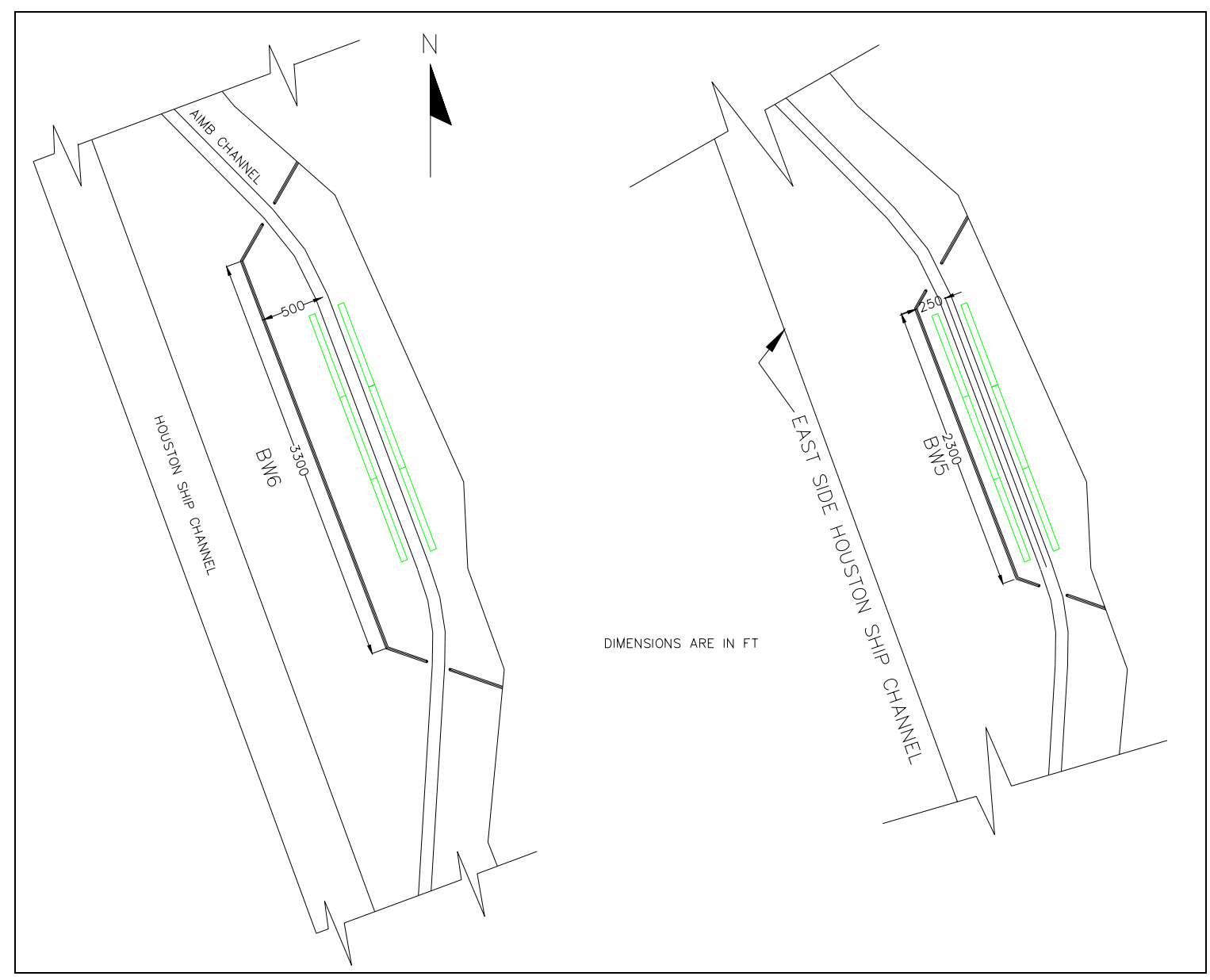

Figure 19. Layout for dikes bw5 and bw6.

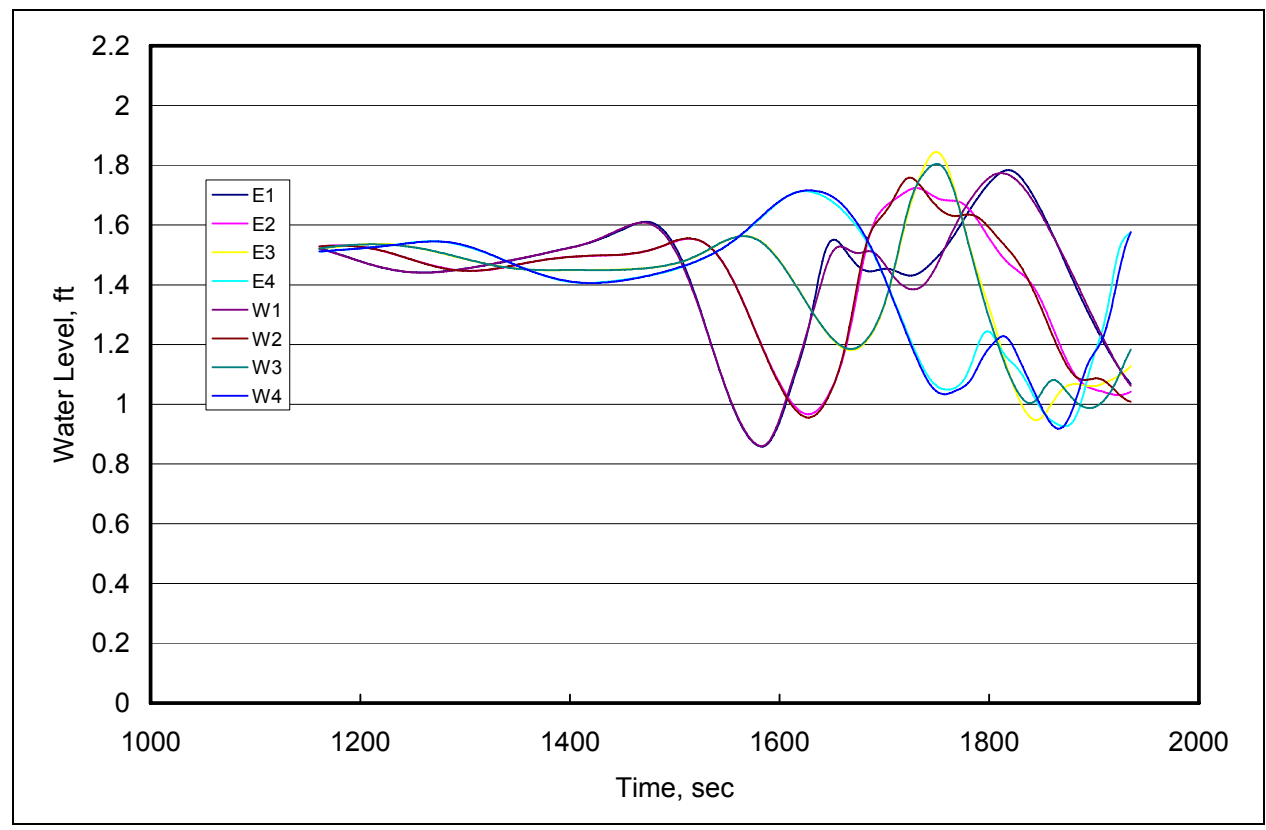

Figure 20. Water levels ( $\mathrm{ft}$ ) in basin with dike bw6 and with tow moored at E3-E4, with SKS Trent outbound 


\section{Dikes bw7 and bw8}

A simulation was performed with a $4000-\mathrm{ft}$ dike having its centerline located $250 \mathrm{ft}$ from the centerline of the AIMB channel. The layout is shown in Figure 21 and the maximum water-level differences are provided in Table 5. No improvement over dike bw6 was observed with this design. The ends of the dike were modified as shown in Figure 21 to try to produce more energy losses at the entrance in an effort to reduce the longitudinal water-level differences. This design was also not as effective as the dike bw6 design in reducing the longitudinal water-level differences. The lateral differences were slightly less than dike bw6 design. Additional changes were needed to reduce the longitudinal ship effects.

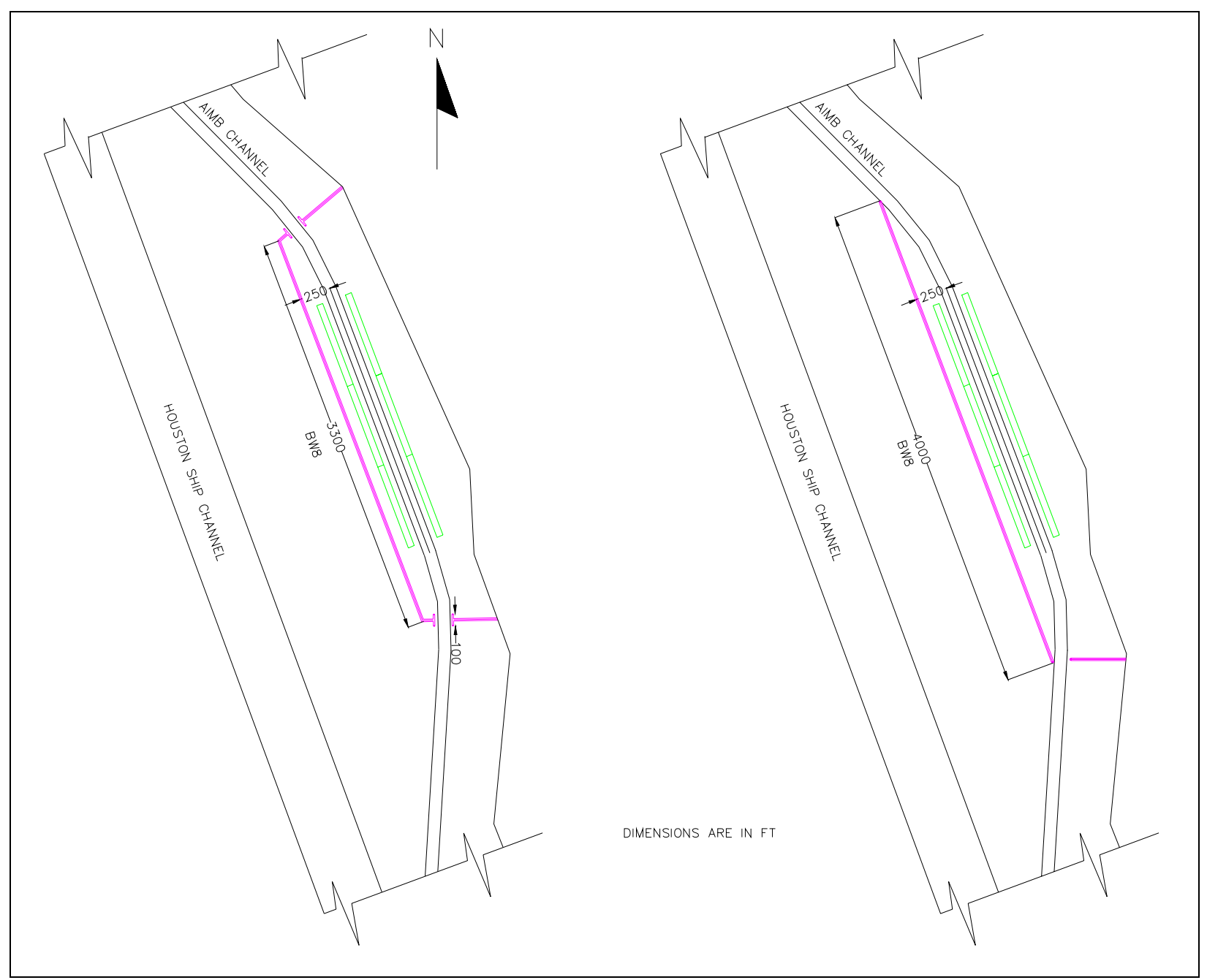

Figure 21. Layout for dikes bw7 and bw8. 


\section{Dike bw9}

The centerline of the dike was moved back to $500 \mathrm{ft}$ from the centerline of the AIMB channel and the end sections were changed as shown in Figure 22. This design was designated dike bw9 and the longitudinal variations of the water levels in the basin were less than those seen with the

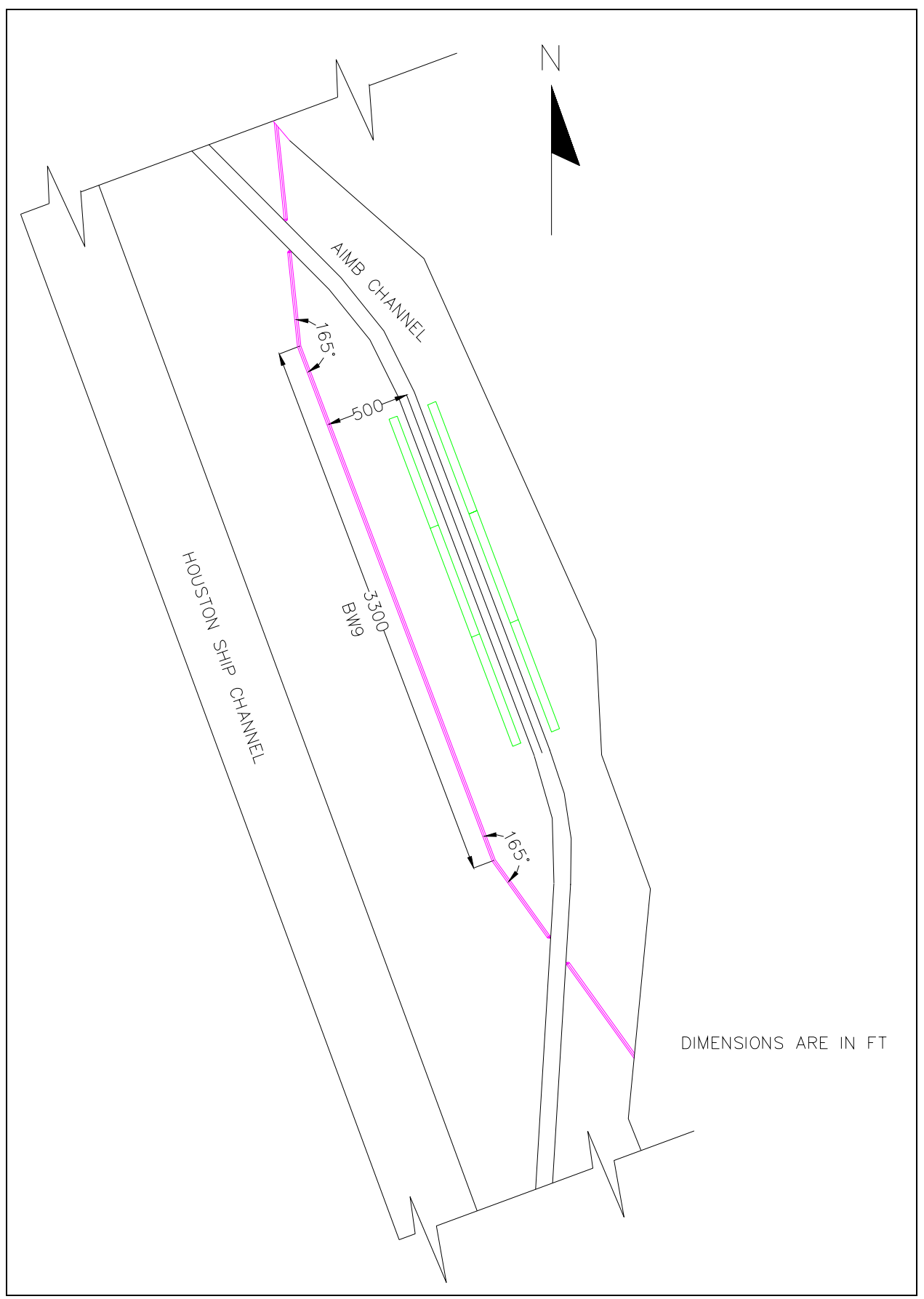

Figure 22. Layout for dike bw9. 
previous dike designs. The maximum longitudinal water-level difference at location $\mathrm{E}_{3}-\mathrm{E}_{4}(0.41 \mathrm{ft})$ was significantly reduced and the maximum lateral water-level difference $(-0.15 \mathrm{ft})$ was just slightly larger than observed with dike bw6. This design provided the minimum water-level variations and smallest longitudinal water-level differences of all previous designs evaluated. There was concern that vessel maneuverability might be difficult owing to the alignment of the end sections. Additional modifycations were made to the end sections to improve vessel maneuverability.

\section{Dike bw10}

The end sections on the east side of dike bw9 were removed to form dike bw10, Figure 23. Both the longitudinal and lateral maximum water-level differences were higher than dike bw9 (see Table 5), although these differences were not considered excessive. Modifications were continued to develop a better end section.

\section{Dike bw11}

An end section was placed on the east side of the AIMB channel as shown in Figure 24 to form dike bw11. This dike layout was considered better for navigation than dike bw9. The water levels in the basin were not significantly different from either the bw9 or bw10 designs. The maximum longitudinal water-level difference at location E3-E4 was lower than bw10 and only slightly higher than bw9, as shown in Table 5. The maximum lateral water-level difference was less than those achieved with both bw9 and bw10. Based on water-level variations in the basin during the ship transit and the end section alignment for vessel maneuverability during entry and exit, this design was considered successful in reducing ship effects for an outbound transit for a ship similar to the SKS Trent. 


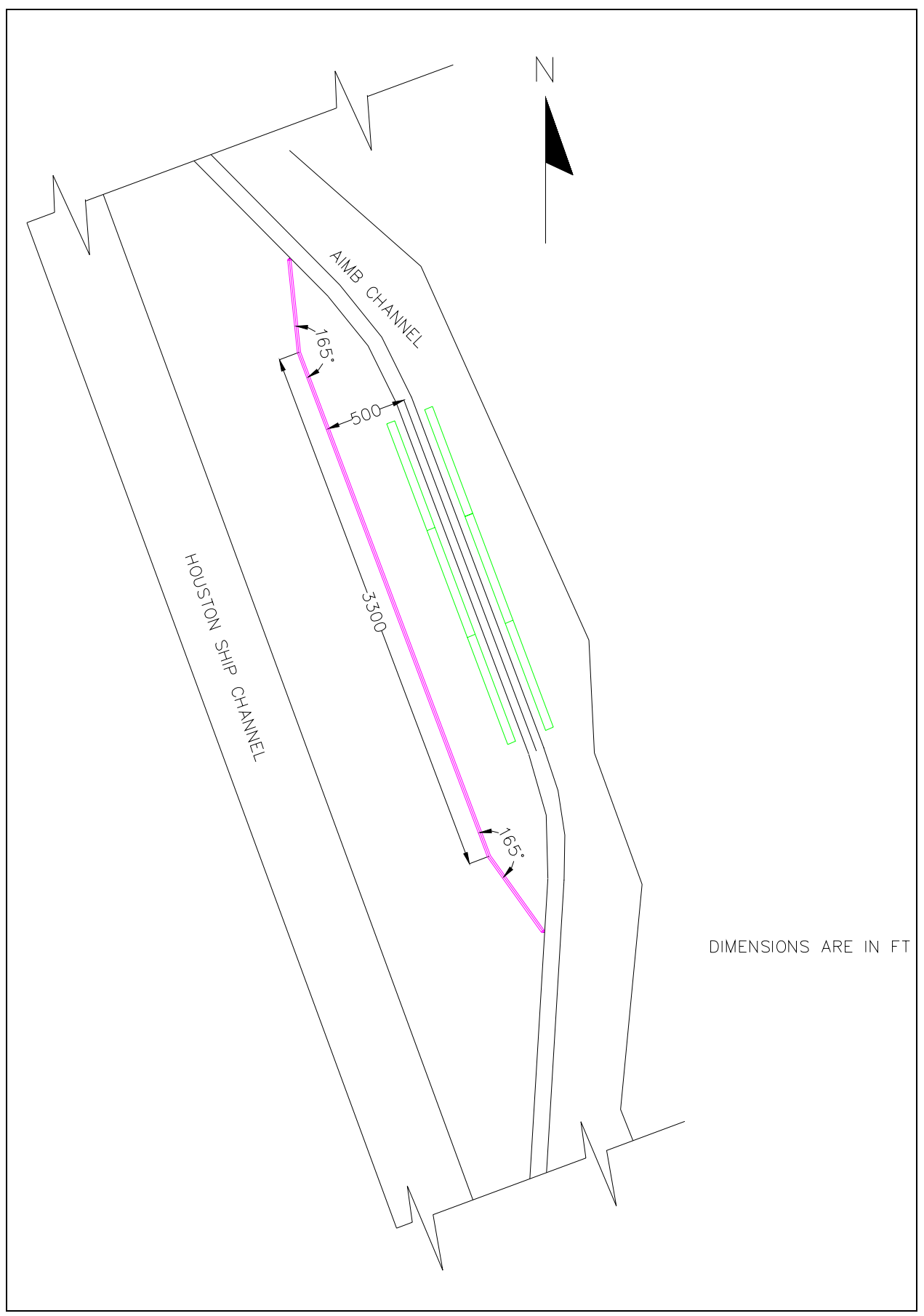

Figure 23. Layout for dike bw10.

\section{Dike bw12}

To determine if the end section design was effective with the smaller dike located closer to the mooring basin, the end section configuration with dike bw11 was placed on the 2300-ft dike located $250 \mathrm{ft}$ from the AIMB channel centerline. This design was designated dike bw12 and is shown in 


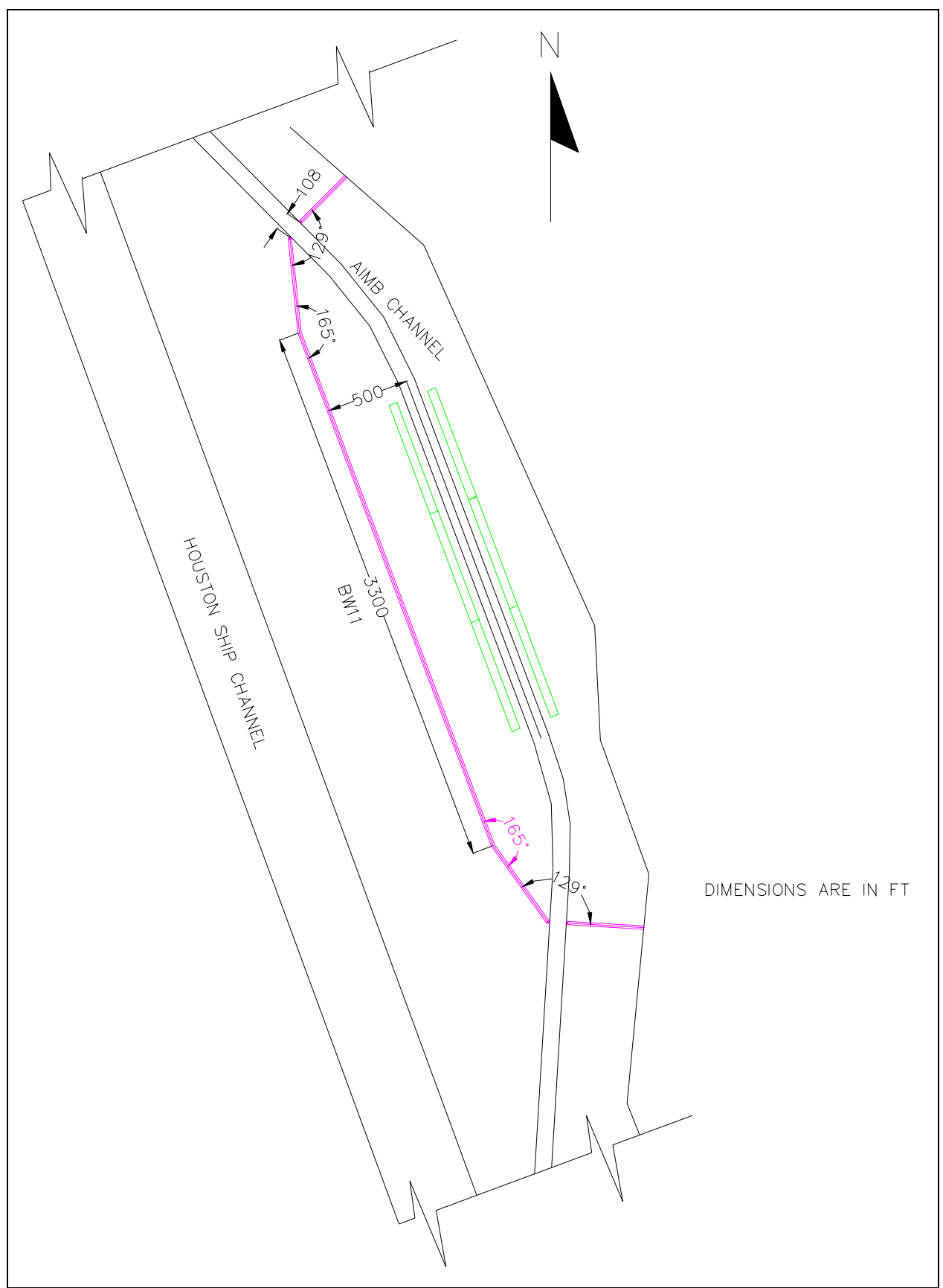

Figure 24. Layout for dike bw11.

Figure 25. Water-level variations in the basin were higher and the maximum longitudinal water-level difference was twice that observed with dike bw11. This design demonstrated the need to have a $3300 \mathrm{ft}$ long dike with its centerline located $500 \mathrm{ft}$ from the AIMB centerline. 


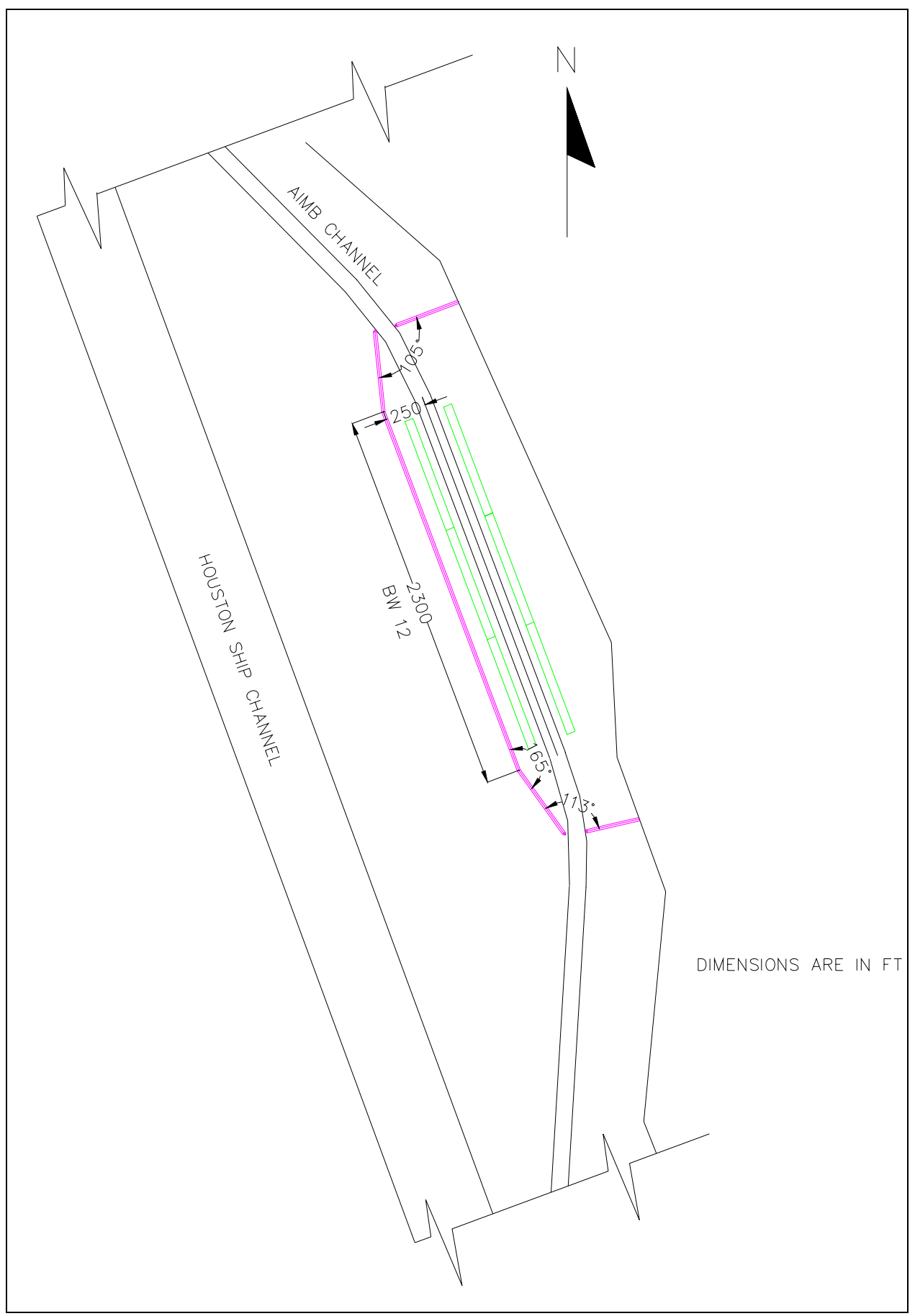

Figure 25. Layout for dike bw12.

\section{Dike bw13}

The opening in the end section was considered small for navigation, so a simulation was performed next with the opening to the basin increased from $108 \mathrm{ft}$ with dike bw11 to $241 \mathrm{ft}$. This design was designated dike bw13 and is shown in Figure 26. The maximum longitudinal water level 


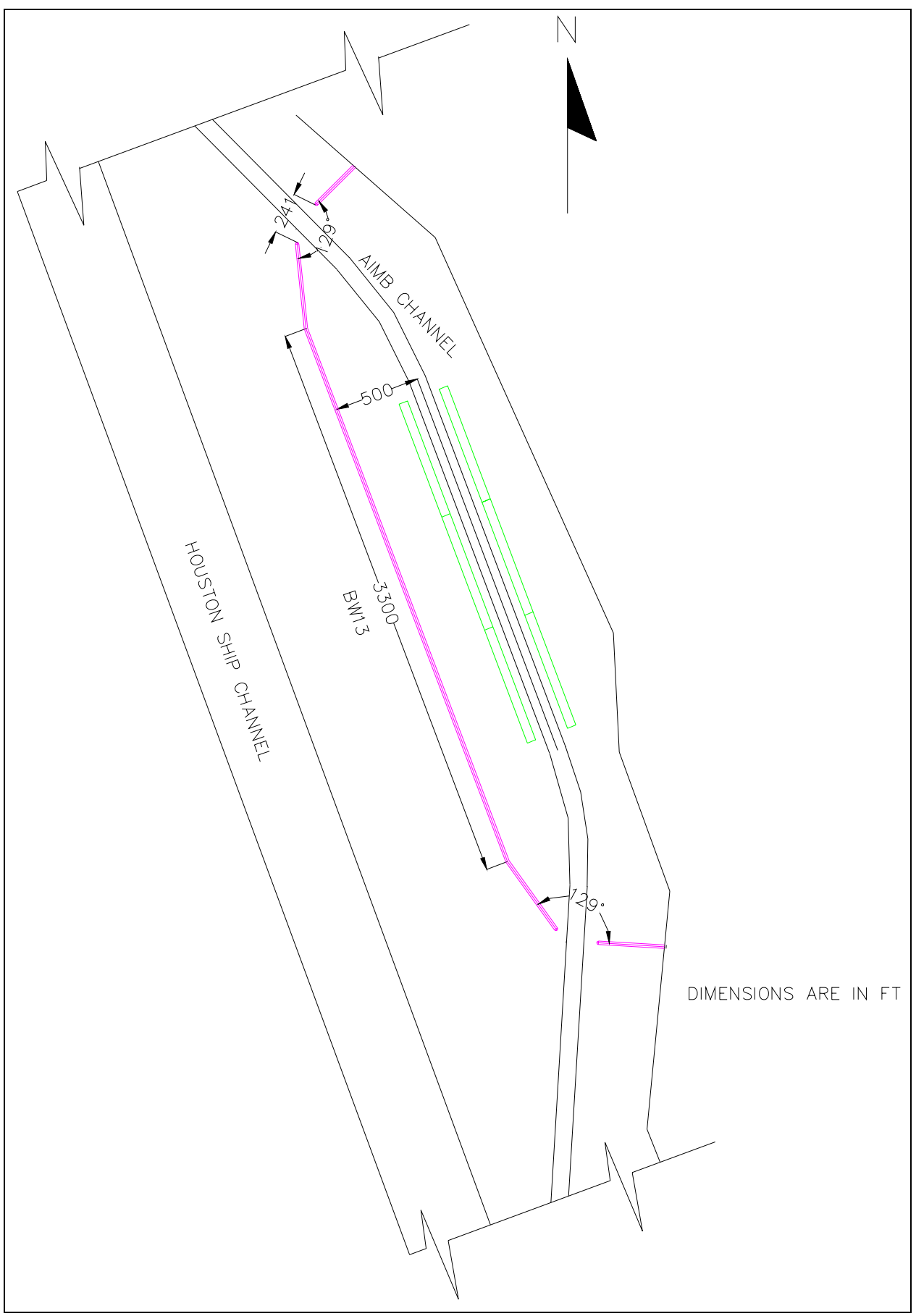

Figure 26. Layout for dike bw13.

difference increased from $0.48 \mathrm{ft}$ observed with dike bw11 to $0.59 \mathrm{ft}$ with dike bw13. The lateral water-level difference increased slightly. This design was considered satisfactory. 


\section{Dike bw14}

A simulation was performed next with the opening to the basin set at $153 \mathrm{ft}$. The north end section on the east side of the AIMB channel was moved $50 \mathrm{ft}$ north and the south end section on the east side of the AIMB channel was moved $75 \mathrm{ft}$ south. These sections were moved so that the centerlines of the end section would intersect the centerline of the AIMB channel at the same location, as shown in Figure 27. The water levels in the basin with this design are shown in Figure 28. Maximum water-level differences shown in Table 5 were reduced from those observed with dike bw13. Based on water-level variations in the basin during the ship transit and the end section alignment for vessel maneuverability during entry and exit, this design was considered successful in reducing ship effects for an outbound transit for a ship similar to the SKS Trent.

\section{Comparison of Designs}

A comparison of the maximum water-level differences for the various dike designs evaluated for the SKS Trent outbound transit is shown in Figure 29. The longitudinal water-level difference is the difference between locations E3 and E4. This was the location where the maximum differences were found. The lateral water-level difference is the difference between $\mathrm{E}_{4}$ and W4. The smallest longitudinal differences occurred with dikes bw9 and bw11; however, these designs were considered difficult to navigate. The lateral differences were all fairly small with a dike in place. Dike bw14 was the design selected for further evaluation. 


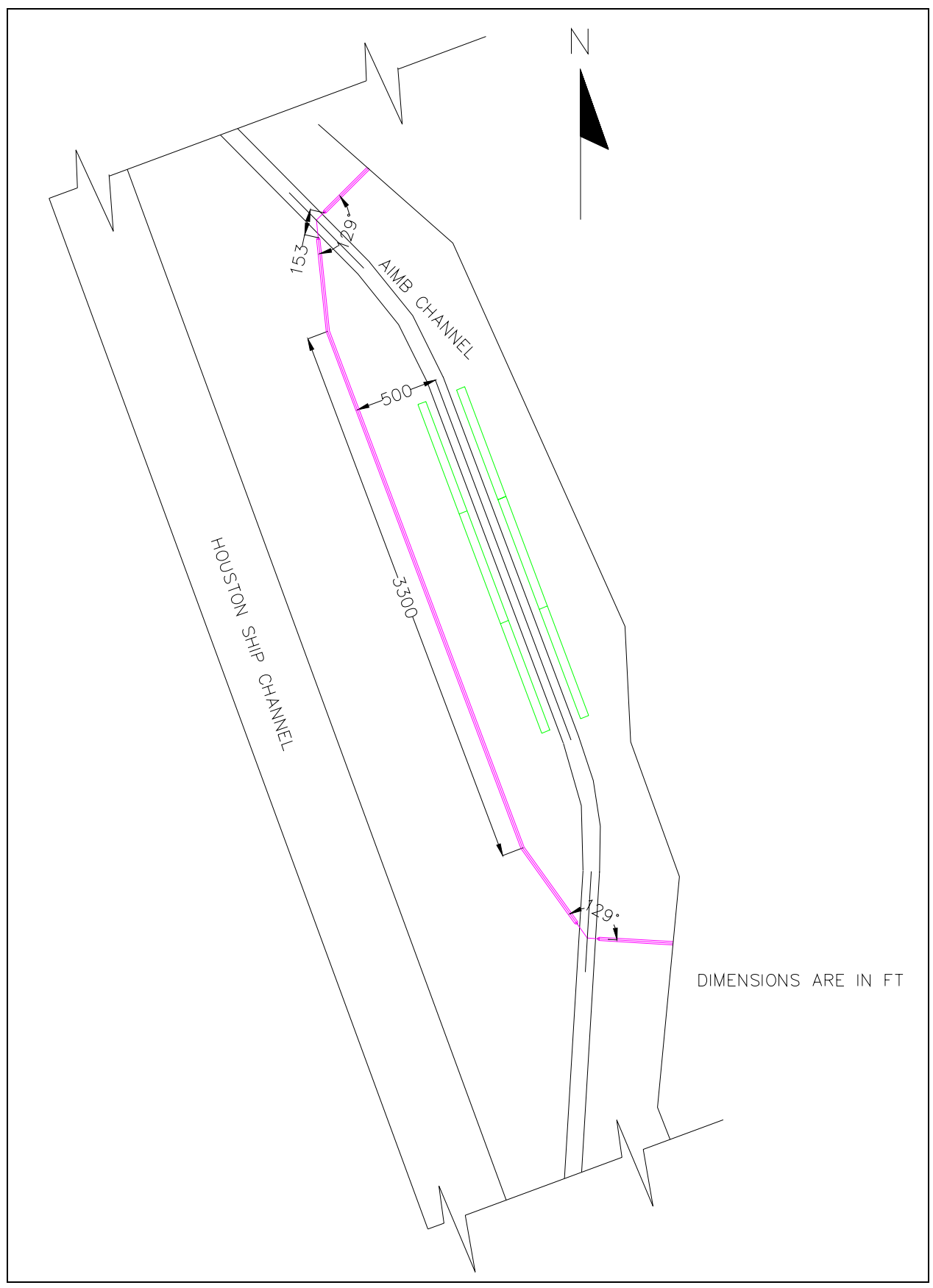

Figure 27. Layout for dike bw14. 


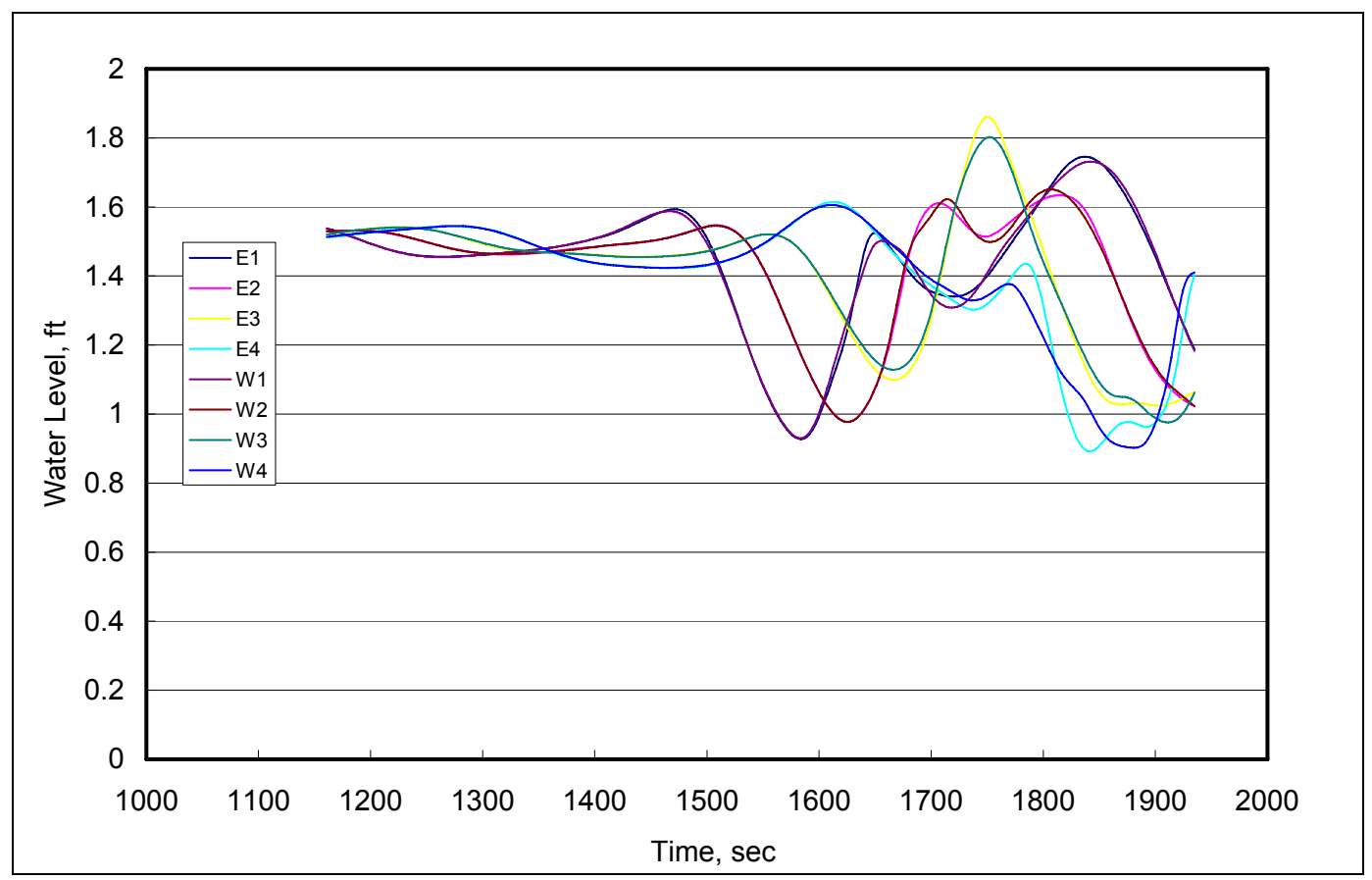

Figure 28. Water levels (ft) in basin with dike bw14 with tow moored at location E3-E4 and SKS Trent outbound.

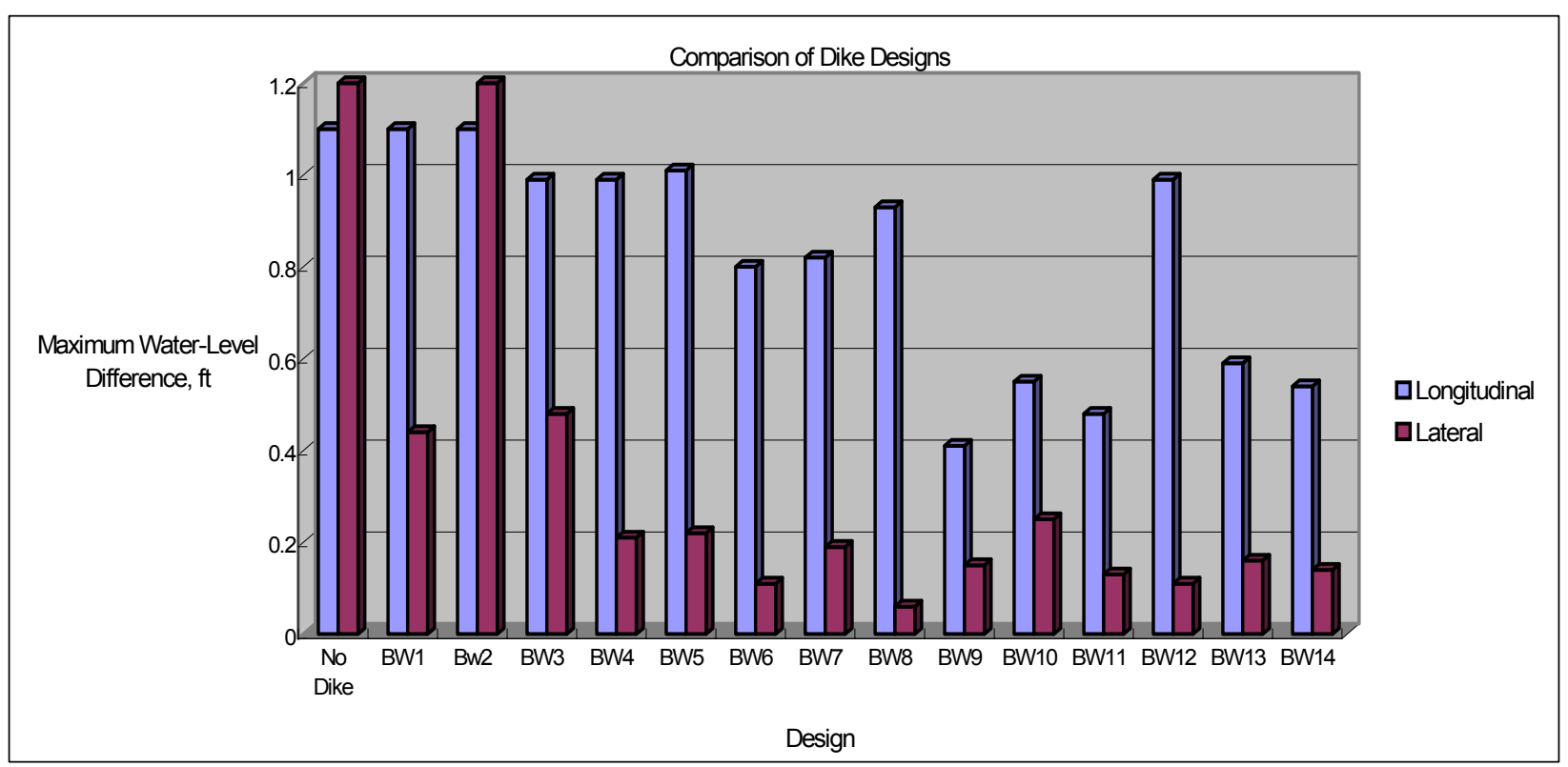

Figure 29. Comparison of maximum water-level differences for the different dike designs with SKS Trent outbound. 


\section{Dike bw14 with Tow Moored in Varying Locations}

Additional simulations were performed with dike bw14 to determine the ship effects with barges moored in different locations within the basin. The ship transit used was the SKS Trent outbound. The barges were moored in locations E3-E4, E2-E3, and E1-E2. Evaluation of previous results had shown the water-level variations were larger on the east side of the AIMB channel. The maximum longitudinal and lateral water-level differences determined for these locations are provided in Table 6. No significant differences were observed for the different mooring locations. The differences computed for location E3-E4 were slightly higher than those at locations E2-E3 and E1-E2.

Table 6. Maximum water-level differences in mooring basin in feet with dike bw14 for different mooring locations with SKS Trent outbound.

\begin{tabular}{|l|l|l|l|l|}
\hline Mooring Location & $\begin{array}{l}\text { Longitudinal } \\
\text { (North to South) }\end{array}$ & Location: time & $\begin{array}{l}\text { Lateral } \\
\text { (East to West) }\end{array}$ & Location:time \\
\hline E3-E4 & 0.54 & E3-E4:1749.2 & -0.14 & E4-W4:1834.4 \\
\hline E2-E3 & 0.51 & E3-E4:1751.8 & -0.13 & E4-W4:1922.1 \\
\hline E1-E2 & 0.51 & E1-E2:1646.0 & -0.13 & E4-W4:1922.1 \\
\hline
\end{tabular}

\section{Dike bw14 with Different Ship Transits}

Simulations were performed next to determine the ship effects in the basin with different ships and types of transits. The ship effects with the adopted speed curves for the Eagle Subaru outbound (Figure 5f) and the Berge Kobe and SKS Trent inbound (Figures $5 \mathrm{~d}$ and $5 \mathrm{~h}$ ) were evaluated. The length, beam, and draft for these ships and the adopted measurements for the simulations are provided in Table 4. The maximum longitudinal and lateral water-level differences for these ship transits are listed in Table 7. The maximum longitudinal difference was at mooring location E1-E2 for the inbound ships. The Berge Kobe inbound caused the highest maximum longitudinal water-level difference $(-0.81 \mathrm{ft})$. The water levels in the basin during this ship transit are shown in Figure 30. The water-surface elevation varied from 0.8 to $2 \mathrm{ft}$ during the transit. The water-surface elevation varied from 0.9 to $1.8 \mathrm{ft}$ with the $S K S$ Trent outbound. 
Table 7. Maximum water-level differences in mooring basin in feet with dike bw14 and barges moored in E3-E4 for different ship transits.

\begin{tabular}{|l|l|l|l|l|}
\hline Ship - Transit & $\begin{array}{l}\text { Longitudinal } \\
\text { (North to South) }\end{array}$ & Location: time & $\begin{array}{l}\text { Lateral } \\
\text { (East to West) }\end{array}$ & Location:time \\
\hline SKS Trent - Outbound & 0.54 & E3-E4:1749.2 & -0.14 & E4-W4:1834.4 \\
\hline Eagle Subaru - Outbound & 0.60 & E1-E2:1460.3 & 0.15 & E4-W4:1604.8 \\
\hline Berge Kobe - Inbound & -0.81 & E1-E2:1452.5 & -0.13 & E1-W1:1609.9 \\
\hline SKS Trent - Inbound & -0.61 & E1-E2:1460.3 & -0.12 & E1-W1:1695.1 \\
\hline
\end{tabular}

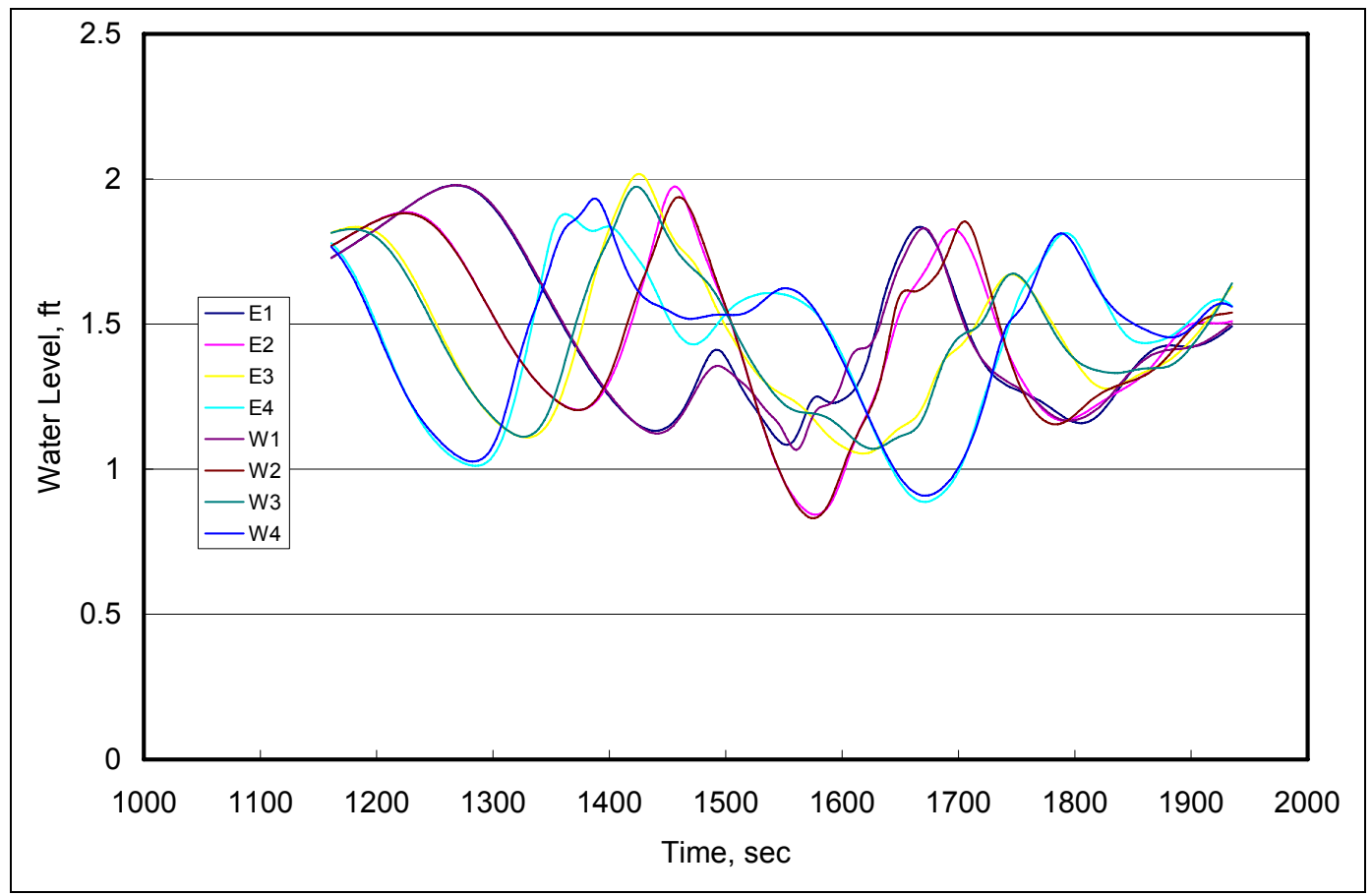

Figure 30. Water levels ( $\mathrm{ft}$ ) in basin with dike bw14, Berge Kobe inbound and tow moored at E1-E2.

\section{Berge Kobe Inbound}

As the highest water-level difference was observed with the Berge Kobe inbound, a simulation was performed without a dike in place to observe the water-level variations in the mooring areas during an inbound transit. The water-surface elevation varied between 0 and $2.3 \mathrm{ft}$ (Figure 31), indicating that dike bw14 was very effective in reducing ship effects in the basin. Table 8 compares the water-level differences with dike bw14 and without a dike. The dike reduced the longitudinal difference by $0.9 \mathrm{ft}$ and the lateral difference by $0.8 \mathrm{ft}$. 


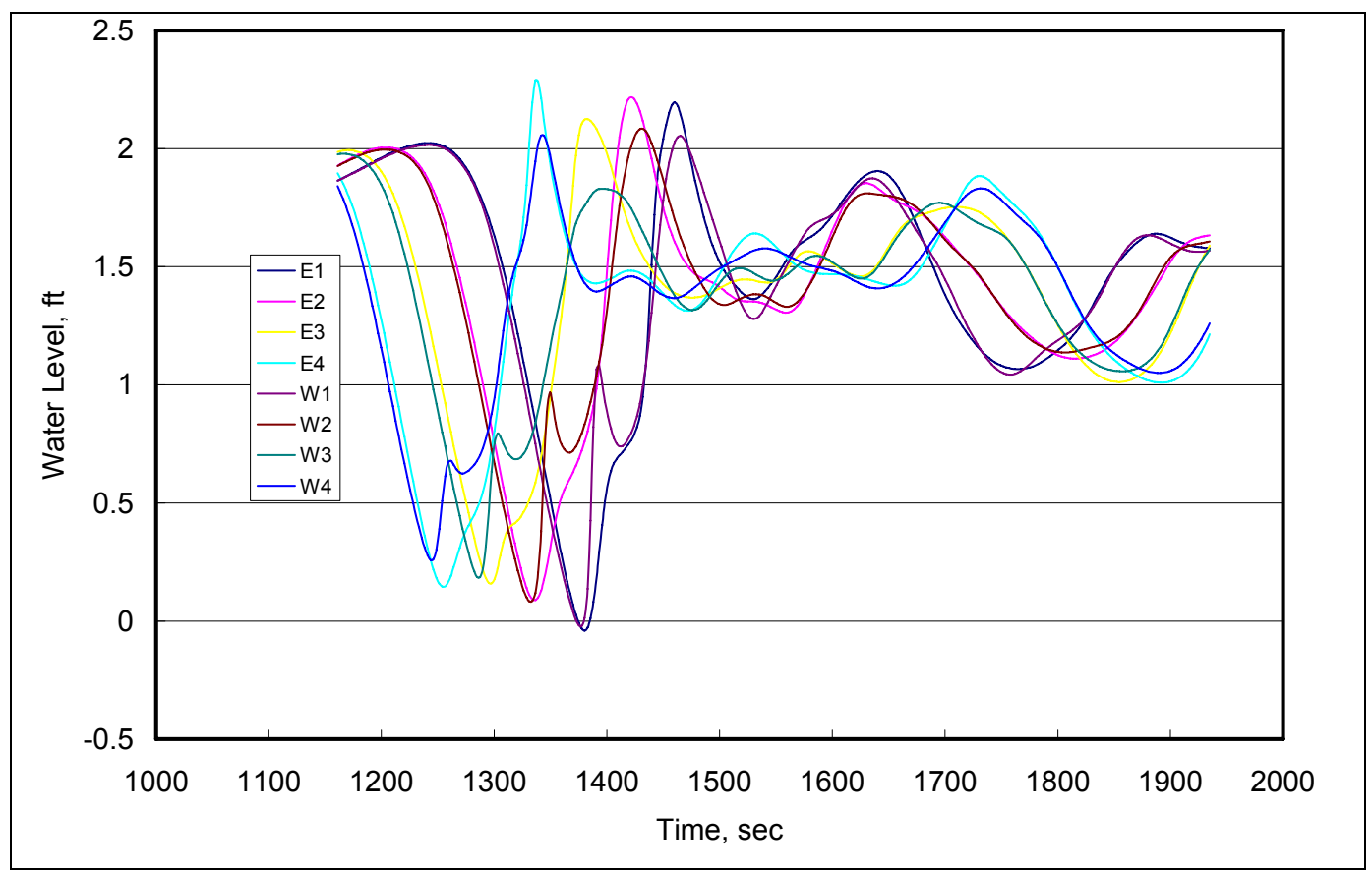

Figure 31. Water levels in basin with no dike and Berge Kobe inbound.

Table 8. Maximum water-level differences in mooring basin in feet without dike and barges moored in E3-E4 with Berge Kobe inbound.

\begin{tabular}{|l|l|l|l|l|}
\hline Ship - Transit & $\begin{array}{l}\text { Longitudinal } \\
\text { (North to South) }\end{array}$ & Location: time & $\begin{array}{l}\text { Lateral } \\
\text { (East to West) }\end{array}$ & Location:time \\
\hline Dike bw14 & -0.81 & E1-E2:1452.5 & -0.13 & E1-W1:1609.9 \\
\hline Without dike & -1.7 & E3-E4:1336.4 & -0.9 & E1-W1:1390.6 \\
\hline
\end{tabular}

\section{Forces in Mooring Lines}

The forces in the lines can be estimated from the water-surface slopes computed in the basin making the following assumptions. The barges act as a single vessel that conforms to the water surface and the forces attributable to drag and inertia are negligible. The longitudinal hawser force, $F_{H}$, is

$$
F_{H}=W \frac{H_{u}-H_{d}}{L_{u d}}
$$


where

$$
\begin{aligned}
W= & \text { weight of barges (also the weight of water displaced by barges) } \\
H_{u}= & \text { water-surface elevation at a point } u \text { near the upstream end of } \\
& \text { the barges } \\
H_{d}= & \text { water-surface elevation at a point } d \text { near the downstream end } \\
& \text { of the barges } \\
L_{u d}= & \text { horizontal distance between points } u \text { and } d .
\end{aligned}
$$

Under these assumptions, the force in the line necessary to hold the barges in place is a function of water-surface slope only. With barge dimensions of $52 \mathrm{ft}$ wide by $700 \mathrm{ft}$ long with a 9 - $\mathrm{ft}$ draft, the weight of this vessel would be $700 \mathrm{ft} \times 52 \mathrm{ft} \times 9 \mathrm{ft} \times 64.3 \mathrm{lb} / \mathrm{ft} 3$ or $21,064,680 \mathrm{lb}(10,532$ tons). The maximum longitudinal slope of the water surface over a $700-\mathrm{ft}$ long mooring area would be the maximum longitudinal water-level difference determined from one end of the mooring location to the other divided by $700 \mathrm{ft}$. For example, without the dike in place, the maximum longitudinal water-level difference determined at mooring location E3-E4 was $1.1 \mathrm{ft}$. The maximum longitudinal force in the mooring line would be $(1.1 \mathrm{ft} / 700 \mathrm{ft}) \times 10,532$ tons $=16.6$ tons. There are lateral forces without the dike in place owing to the water-surface slope in this direction. Under the same assumptions as with the longitudinal force, the maximum lateral force in the mooring line would be $1.2 \mathrm{ft} / 300 \mathrm{ft} \times 10,532$ tons $=42.1$ tons, significantly higher than the longitudinal force. These forces are estimates, as the actual force would be the resultant of the longitudinal and lateral forces. The maximum longitudinal and lateral forces computed in the manner described above with dike bw14 in place are 8.1 and 4.9 tons, respectively. The dike is definitely beneficial in reducing the forces in the mooring lines. The maximum hawser forces computed from the maximum longitudinal and lateral water-level differences in Table 5 and Figure 29 are shown in Figure 32. Time histories of the computed longitudinal and lateral hawser forces for without dike and dike bw14 conditions are shown in Figure 33. 


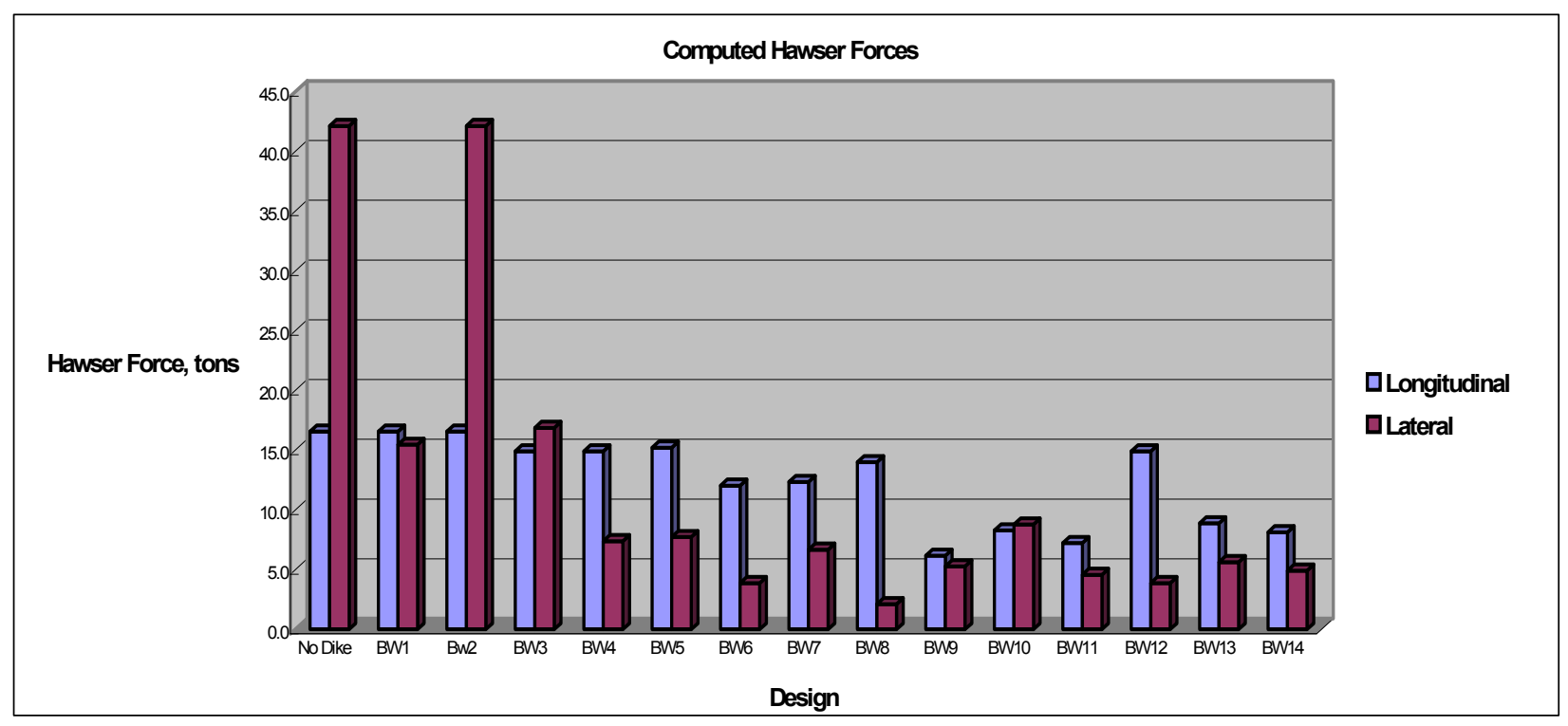

Figure 32. Computed hawser forces for the different dike designs, SKS Trent outbound.

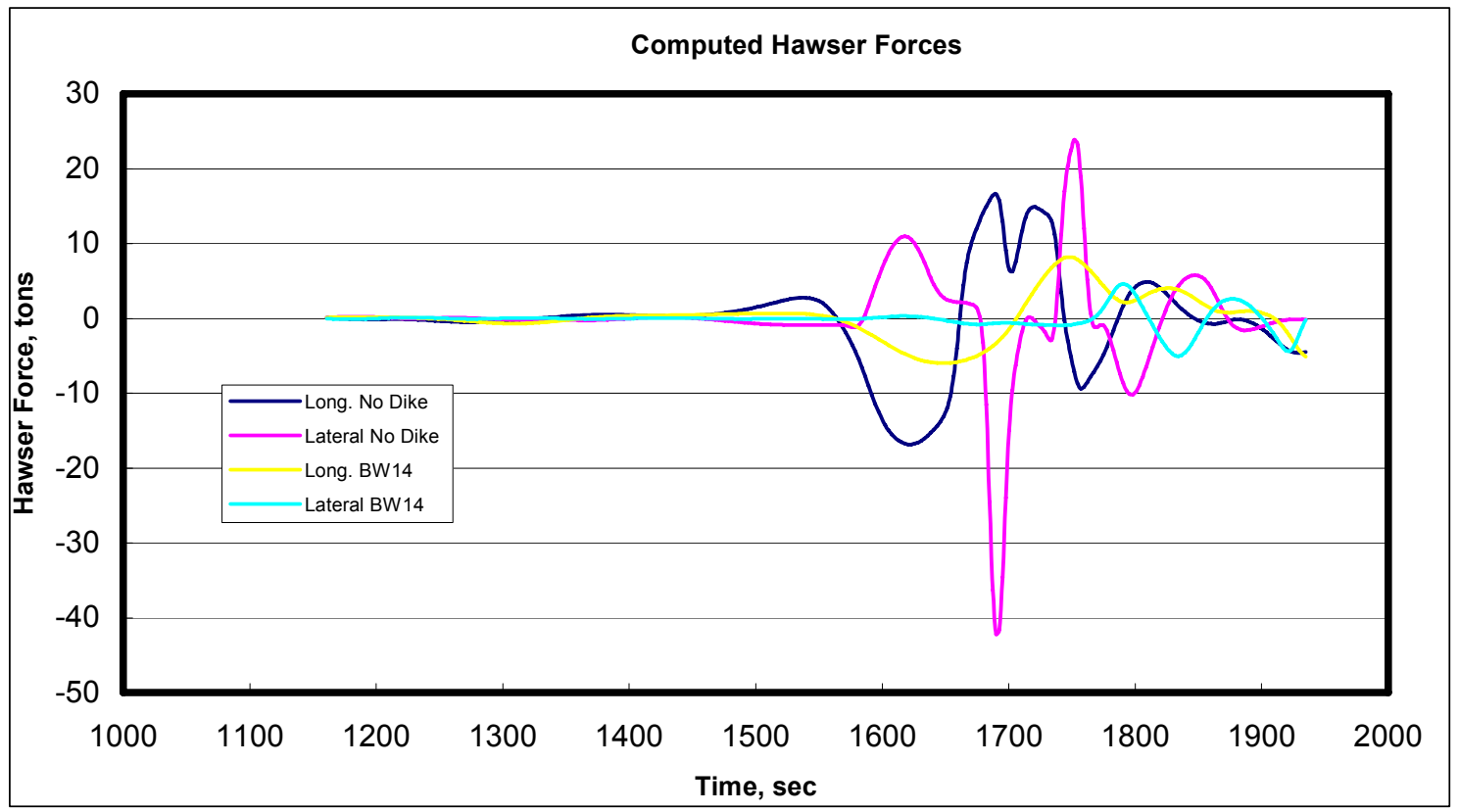

Figure 33. Time histories of computed hawser forces for the without dike and dike bw14 conditions, SKS Trent outbound. 


\section{Summary and Recommendations}

Comparison of the water-level data from the field experiments and the water levels computed for existing conditions using the numerical model HIVEL2D showed the model to be a viable tool for developing a dike design to help reduce ship effects in the Atkinson Island Mooring Basin. After validation of the model for existing conditions, the new channel for the AIMB was added to the model along with the proposed mooring areas on the east and west sides of the channel. Several dike designs were evaluated based on the water-level variations in the mooring basin during ship transits. A dike design that provided the smallest water-level variations during the transits was the ultimate goal.

The presence of a longitudinal dike helped reduce the east-west (lateral) variations in the water level during the ship transits. Locating the centerline of the dike $500 \mathrm{ft}$ from the centerline of the straight section further reduced the east-west water-level variations as well as reducing the northsouth (longitudinal) water-level variations. End sections with openings on the north and south ends of the basin for the channel were needed to further reduce the longitudinal variations.

Dike bw9, Figure 22, was the most effective in reducing the longitudinal water-level variations in the basin, but might be difficult for navigation. Dike bw14 was considered the most effective design based on water-level variations and navigability. This design will help provide protection from ship waves for the barges moored in the basin and reduce the forces in the hawser lines holding the barges in place.

Simulations with different ship transits indicated that the longitudinal water-level variations were the largest in the southeast corner of the basin, mooring location E3-E4, with outbound ships. The largest longitudinal water-level variations in the basin with dike bw14 and inbound ships occurred at mooring location E1-E2. The inbound ships evaluated produced slightly larger longitudinal water-level variations than the outbound ships. 
Estimation of the forces in the mooring lines, hawser forces, showed that the forces were reduced significantly with dike bw14 in place, especially the lateral hawser forces. Dike bw14 is recommended for the AIMB to help reduce the water-level variations in the basin caused by ship traffic in the Houston Ship Channel. This design could be constructed in stages to observe actual field performance. The 3300-ft dike with no end sections, bw4, reduced the lateral variations in water level significantly, and reduced the longitudinal differences by $0.1 \mathrm{ft}$ from the no dike condition. The end sections were needed to reduce longitudinal water-level differences. Adding end sections on the west side of the basin, bw10, reduced the longitudinal water-level differences by $0.55 \mathrm{ft}$ from the no dike condition. With end sections on both sides of the AIMB channels, bw14, the longitudinal water-level difference was reduced by $0.56 \mathrm{ft}$ from the no dike condition. Dike bw14 was more effective in reducing the lateral water-level differences than dike bw10. 


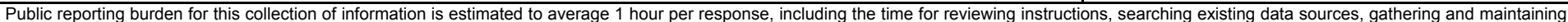

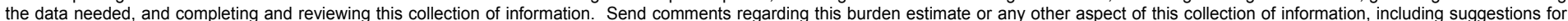

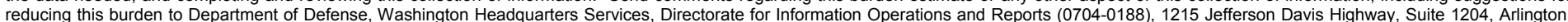

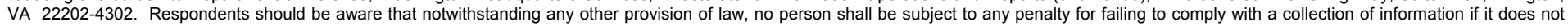
display a currently valid OMB control number. PLEASE DO NOT RETURN YOUR FORM TO THE ABOVE ADDRESS.
1. REPORT DATE (DD-MM-YYYY) September 2006

$$
\text { Final report }
$$

4. TITLE AND SUBTITLE

Atkinson Island Mooring Basin Alternatives, Houston Ship Channel
3. DATES COVERED (From - To)

5a. CONTRACT NUMBER

5b. GRANT NUMBER

5c. PROGRAM ELEMENT NUMBER

\section{AUTHOR(S)}

Stephen T. Maynord, John E. Hite, Jr., and Mario J. Sanchez

5d. PROJECT NUMBER

5e. TASK NUMBER

\section{5f. WORK UNIT NUMBER}

8. PERFORMING ORGANIZATION REPORT NUMBER

ERDC/CHL TR-06-19

U.S. Army Engineer Research and Development Center

Coastal and Hydraulics Laboratory

3909 Halls Ferry Road

Vicksburg, MS 39180-6199

\section{SPONSORING / MONITORING AGENCY NAME(S) AND ADDRESS(ES)}

U.S. Army Engineer District, Galveston

PO Box 1229

Galveston, TX 77553-1229

10. SPONSOR/MONITOR'S ACRONYM(S)

11. SPONSOR/MONITOR'S REPORT NUMBER(S)

\section{DISTRIBUTION / AVAILABILITY STATEMENT}

Approved for public release; distribution is unlimited.

\section{SUPPLEMENTARY NOTES}

\section{ABSTRACT}

The U.S. Army Engineer District, Galveston (SWG), is investigating methods to reduce ship effects in the Atkinson Island Mooring Basin (AIMB) from ship traffic in the Houston Ship Channel. The Engineer Research and Development Center (ERDC) assisted SWG in this investigation. A field study was conducted first to gain an understanding of the ship effects that are causing mooring problems in the AIMB and collect ship effects data to be used to validate the numerical model HIVEL2D to the existing conditions in the Houston Ship Channel. The numerical model successfully reproduced the results from the field experiments and was then used to evaluate dike designs with the new AIMB channel. Several dike designs were evaluated and a 3,300-ft-long dike with the centerline of the dike located $500 \mathrm{ft}$ west of the centerline of the AIMB channel was found to be effective in minimizing ship effects in the basin. End sections placed on the dike with a 150-ft opening at the entrances to the basin further reduced the water level variations inside the basin during ship transits. The dike design bw14 included these features and was recommended for installation at the project.

\section{SUBJECT TERMS}

Dike design

Field experiments
Hawser forces

Houston Ship Channel

Mooring basin design

16. SECURITY CLASSIFICATION OF:

a. REPORT

UNCLASSIFIED

b. ABSTRACT
UNCLASSIFIED

UNCLASSIFIED c. THIS PAGE
Numerical modeling

Ship effects

\begin{tabular}{|c|c|}
\hline $\begin{array}{l}\text { 17. LIMITATION } \\
\text { OF ABSTRACT }\end{array}$ & $\begin{array}{c}\text { 18. NUMBER } \\
\text { OF PAGES } \\
63\end{array}$ \\
\hline
\end{tabular}

19a. NAME OF RESPONSIBLE PERSON

19b. TELEPHONE NUMBER (include area code) 